\title{
Catalytic Atroposelective Dynamic Kinetic Resolution of Biaryl Lactones with Activated Isocyanides
}

Linghui Qian, ${ }^{\dagger,}$ Ling-Fei Tao, ${ }^{\dagger}{ }^{\S}$ Wen-Tao Wang, ${ }^{\dagger}$ Ehtesham Jameel, ${ }^{\dagger}$ Zhang-Hong Luo, ${ }^{\dagger}$ Tao Zhang, "Yu Zhao, " and Jia-Yu Liao*,†,

\section{Supporting Information}

Table of contents

Page

I. General information

S2

II. Ag-catalyzed atroposelective DKR of $\mathbf{1}$ with $\mathbf{2}$

S3

III. Characterization of compounds 3 and $\mathbf{4}$

S3

IV. DFT calculations

S30

V. Gram-scale synthesis of $\mathbf{3 a}$

VI. Synthetic transformations

S40

VII. X-ray crystallographic analysis and determination of configuration of $\mathbf{3 a}$

S49

VIII. References

IX. NMR spectra of the products 


\section{General information}

${ }^{1} \mathbf{H}$ and ${ }^{13} \mathbf{C}$ NMR spectra were recorded on a Bruker AVIII $500 \mathrm{M}(500 \mathrm{MHz})$ or WNMR-I 400M (400 MHz) spectrometer. Chemical shifts were reported in parts per million (ppm), and the residual solvent peak was used as an internal reference: ${ }^{1} \mathrm{H}$ (chloroform $\delta$ 7.26; DMSO $\delta 2.50$ ), ${ }^{13} \mathrm{C}$ (chloroform $\delta 77.16$; DMSO $\delta 39.52$ ). Data are reported as follows: chemical shift, multiplicity $(\mathrm{s}=$ singlet, $\mathrm{d}=$ doublet, $\mathrm{t}=$ triplet, $\mathrm{q}=$ quartet, $\mathrm{m}=$ multiplet, $\mathrm{br}=$ broad, $\mathrm{dd}=$ doublet of doublets), coupling constants (Hz) and integration. Melting point (MP) was obtained on Buchi M-560. For thin layer chromatography (TLC), Huanghai TLC plates (HSGF 254) were used, and compounds were visualized with a UV light at $254 \mathrm{~nm}$. High resolution mass spectra (HRMS) were obtained on an Agilent G6545 spectrometer using an electron spray ionization time-of flight (ESI-TOF) source. X-ray diffraction analysis was performed on a Bruker D8 Venture diffractometer. Optical rotations were recorded on an IP-digi 300 automatic polarimeter. Enantiomeric excesses (ee) were determined by HPLC analysis on an Agilent HPLC 1260 Infinity II; column, Chiralpak IA, IB N-5, IC, and IE.

Unless otherwise noted, all reactions were carried out under an ambient atmosphere; exclusion of air or moisture was not required. Anhydrous and deuterated solvents were purchased from commercial suppliers and used as received without further purification. Ethyl isocyanoacetate (2a), methyl isocyanoacetate (2b), tert-butyl isocyanoacetate (2c) and $p$-toluenesulfonylmethyl isocyanide (2d) were purchased from commercial suppliers and used without further purification. Isocyanoacetamide (2e) ${ }^{1}$ and all biaryl lactones ${ }^{2}$ were prepared according to literature procedures. Chiral ligands L1-L3 were purchased from commercial suppliers and used directly without further purification. Chiral ligands L4-L6 are known compounds and were prepared according to literature procedures. ${ }^{3}$ Other chemicals were purchased from commercial suppliers and used as received without further purification. 


\section{Ag-catalyzed atroposelective DKR of 1 with 2}

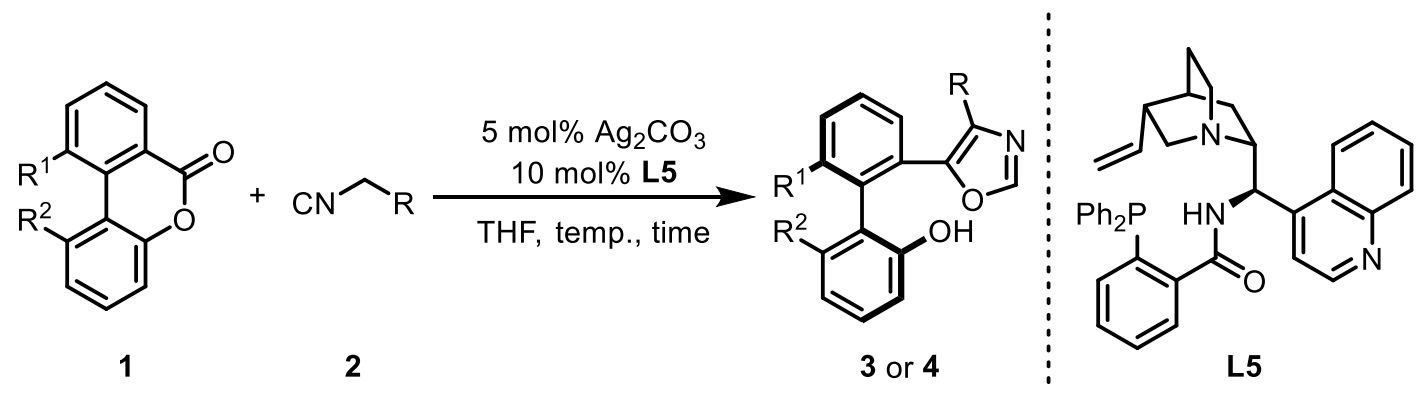

General procedure. To a $10 \mathrm{~mL}$ vial charged with $\mathbf{L 5}(5.8 \mathrm{mg}, 0.010 \mathrm{mmol})$ and $\mathrm{Ag}_{2} \mathrm{CO}_{3}(1.4 \mathrm{mg}, 0.005 \mathrm{mmol})$ was added anhydrous THF. The mixture was stirred at ambient temperature for $5 \mathrm{~min}$, then biaryl lactone $1(0.10 \mathrm{mmol})$ was added. After the mixture was cooled to the given temperature, activated isocyanide $\mathbf{2}$ was added in one portion. The reaction mixture was stirred at the given temperature for the given time, concentrated and purified by flash chromatography (PE/EtOAc) to afford the product 3 or 4 .

Racemic sample for the standard of chiral HPLC spectra was prepared using $10 \mathrm{~mol} \%$ $\mathrm{Ag}_{2} \mathrm{CO}_{3}$ and $20 \mathrm{~mol} \% \mathrm{PPh}_{3}$ as catalyst.

\section{Characterization of compounds 3 and 4}

\section{Ethyl}

(S)-5-(1-(2-hydroxy-4,6-dimethylphenyl)naphthalen-2-yl)oxazole-4-carboxylate<smiles>CCOC(=O)c1ncoc1-c1ccc2ccccc2c1-c1c(C)cc(C)cc1O</smiles>

$3 a$

The general procedure outlined above was followed $(0.10 \mathrm{mmol}$ of $\mathbf{2 a}, 1.0 \mathrm{~mL}$ of THF at $-20{ }^{\circ} \mathrm{C}$ for $48 \mathrm{~h}$ ). The crude reaction mixture was purified by flash column chromatography (PE/EtOAc 2:1). White solid, $35.6 \mathrm{mg}, 92 \%$ yield. MP: $171-172{ }^{\circ} \mathrm{C}$; ${ }^{1} \mathbf{H}$ NMR $\left(500 \mathrm{MHz}, \mathrm{CDCl}_{3}\right): \delta 7.99(\mathrm{~d}, J=8.5 \mathrm{~Hz}, 1 \mathrm{H}), 7.95(\mathrm{~d}, J=8.2 \mathrm{~Hz}, 1 \mathrm{H})$, $7.68(\mathrm{~s}, 1 \mathrm{H}), 7.63-7.54(\mathrm{~m}, 2 \mathrm{H}), 7.51-7.41(\mathrm{~m}, 2 \mathrm{H}), 6.61(\mathrm{~s}, 1 \mathrm{H}), 6.58(\mathrm{~d}, J=0.4 \mathrm{~Hz}$, 
1H), 4.96 (br s, 1H), 4.35-4.19 (m, 2H), $2.28(\mathrm{~s}, 3 \mathrm{H}), 1.82(\mathrm{~s}, 3 \mathrm{H}), 1.25(\mathrm{t}, J=7.1 \mathrm{~Hz}$, $3 \mathrm{H}) ;{ }^{13} \mathbf{C}$ NMR (126 MHz, $\left.\mathrm{CDCl}_{3}\right): \delta 161.9,156.2,153.5,150.1,139.4,138.1,135.1$, $134.6,132.5,128.6,128.5,128.4,127.9,127.7,127.5,126.2$, 126.2, 123.0, 120.6, 114.1, 61.5, 21.3, 19.8, 14.1; HRMS (ESI): m/z calcd. for $\left[\mathrm{C}_{24} \mathrm{H}_{21} \mathrm{NNaO}_{4}, \mathrm{M}+\mathrm{Na}\right]^{+}$: 410.1363; found: 410.1364 .

Optical Rotation: $[\alpha]^{25}=+57.3(\mathrm{c}=0.3$, THF $)$. The absolute configuration of 3a was unambiguously assigned by single crystal X-ray analysis. 92\% ee (HPLC condition: Chiralpak IB N-5 column, $n$-hexane $/ i-\mathrm{PrOH}=90: 10$, flow rate $=1 \mathrm{ml} / \mathrm{min}$, wavelength $=254 \mathrm{~nm}, t_{R}=10.4 \mathrm{~min}$ for minor isomer, $t_{R}=12.3 \mathrm{~min}$ for major isomer).

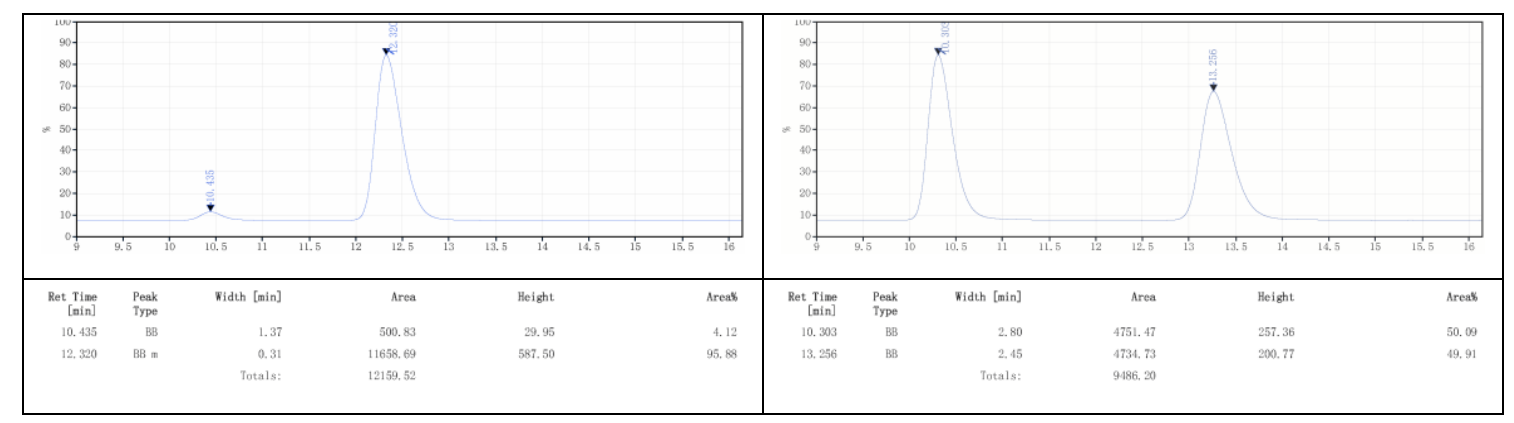

Ethyl

(S)-5-(1-(6-hydroxy-2,3,4-trimethylphenyl)naphthalen-2-yl)oxazole-4-carboxylate (3b)<smiles>CCOC(=O)c1ncoc1-c1ccc2ccccc2c1-c1c(O)cc([N+](=O)[O-])c(C)c1C</smiles>

3b

The general procedure outlined above was followed $(0.1 \mathrm{mmol}$ of $\mathbf{2 a}, 1.0 \mathrm{~mL}$ of THF at $15{ }^{\circ} \mathrm{C}$ for $24 \mathrm{~h}$ ). The crude reaction mixture was purified by flash column chromatography (PE/EtOAc 2:1). Colourless wax, $28.1 \mathrm{mg}, 70 \%$ yield. ${ }^{1} \mathbf{H}$ NMR (500 $\left.\mathrm{MHz} \mathrm{CDCl}_{3}\right): \delta 8.00(\mathrm{~d}, J=8.5 \mathrm{~Hz}, 1 \mathrm{H}), 7.96(\mathrm{~d}, J=8.2 \mathrm{~Hz}, 1 \mathrm{H}), 7.68(\mathrm{~s}, 1 \mathrm{H})$, 7.65-7.55 (m, 2H), 7.51-7.41 (m, 2H), $6.61(\mathrm{~s}, 1 \mathrm{H}), 4.64$ (br s, 1H), 4.34-4.22 (m, 2H), $2.26(\mathrm{~s}, 3 \mathrm{H}), 2.09(\mathrm{~s}, 3 \mathrm{H}), 1.77(\mathrm{~s}, 3 \mathrm{H}), 1.25(\mathrm{t}, J=7.1 \mathrm{~Hz}, 3 \mathrm{H}) ;{ }^{13} \mathbf{C}$ NMR (126 MHz, $\left.\mathrm{CDCl}_{3}\right): \delta 161.9,156.3,150.9,150.1,137.9,136.1,136.1,134.6,132.7,128.6,128.5$, 
$128.4,127.9,127.7,127.5,127.1,126.5,126.2,121.3,115.0,61.5,21.0,17.6,15.3$, 14.2; HRMS (ESI): $\mathrm{m} / \mathrm{z}$ calcd. for $\left[\mathrm{C}_{25} \mathrm{H}_{23} \mathrm{NNaO}_{4}, \mathrm{M}+\mathrm{Na}\right]^{+}$: 424.1519; found: 424.1521 .

Optical Rotation: $[\alpha]^{25} \mathrm{D}=+44.0(\mathrm{c}=0.05$, THF). The absolute configuration of $\mathbf{3 b}$ was assigned by analogy to 3a. 83\% ee (HPLC condition: Chiralpak IB N-5 column, $n$-hexane $/ i-\mathrm{PrOH}=90: 10$, flow rate $=1 \mathrm{ml} / \mathrm{min}$, wavelength $=254 \mathrm{~nm}, \mathrm{t}_{\mathrm{R}}=11.2 \mathrm{~min}$ for minor isomer, $t_{R}=13.8$ min for major isomer).

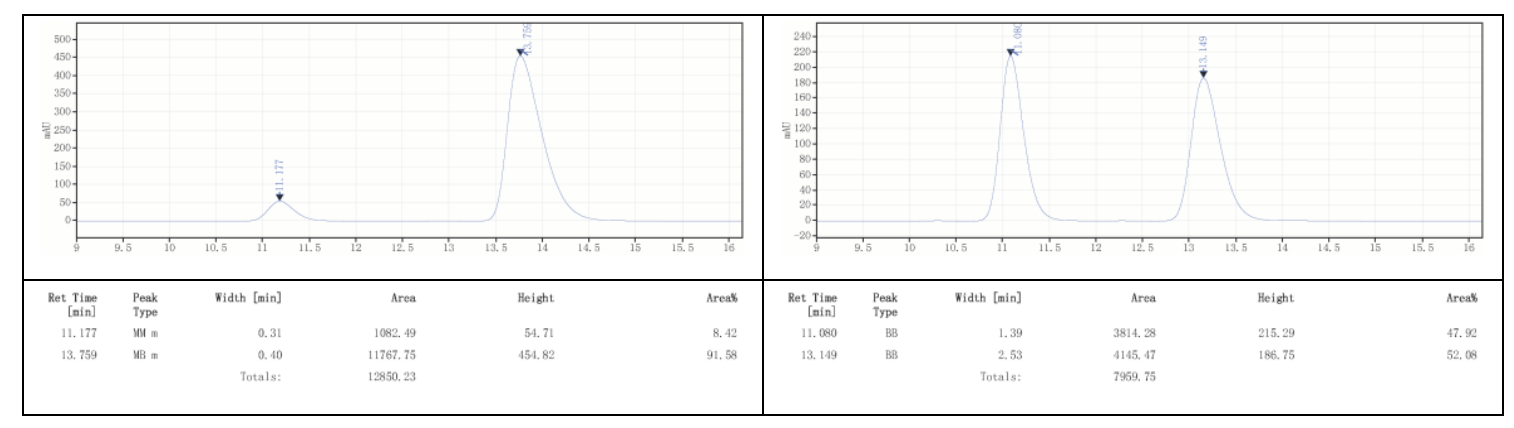

\section{Ethyl}

(S)-5-(1-(6-hydroxy-3-methoxy-2,4-dimethylphenyl)naphthalen-2-yl)oxazole-4-ca rboxylate (3c)<smiles>CCOC(=O)c1ncoc1-c1ccc2ccccc2c1-c1c(O)cc(OC)c(OC)c1OC</smiles>

The general procedure outlined above was followed $(0.12 \mathrm{mmol}$ of $\mathbf{2 a}, 1.0 \mathrm{~mL}$ of THF at $-20{ }^{\circ} \mathrm{C}$ for $48 \mathrm{~h}$ ). The crude reaction mixture was purified by flash column chromatography (PE/EtOAc 2:1). White solid, $35.4 \mathrm{mg}, 85 \%$ yield. MP: $165-166{ }^{\circ} \mathrm{C}$; ${ }^{1} \mathbf{H}$ NMR (400 MHz, $\left.\mathrm{CDCl}_{3}\right): \delta 8.01(\mathrm{~d}, J=8.5 \mathrm{~Hz}, 1 \mathrm{H}), 7.96(\mathrm{~d}, J=8.2 \mathrm{~Hz}, 1 \mathrm{H})$, $7.68(\mathrm{~s}, 1 \mathrm{H}), 7.64-7.55(\mathrm{~m}, 2 \mathrm{H}), 7.53-7.41(\mathrm{~m}, 2 \mathrm{H}), 6.59(\mathrm{~s}, 1 \mathrm{H}), 4.68$ (br s, 1H), 4.36-4.19 (m, 2H), 3.64 (s, 3H), 2.27 (s, 3H), 1.81 (s, 3H), $1.26(\mathrm{t}, J=7.1 \mathrm{~Hz}, 3 \mathrm{H})$; ${ }^{13}$ C NMR (101 MHz, $\left.\mathrm{CDCl}_{3}\right): \delta 161.9,156.1,150.7,150.1,149.4,135.0,134.7$, $132.4,132.2,130.8,128.8,128.7,128.5,128.0,127.7,126.3,126.2,122.3,115.8$, 61.6, 60.2, 16.3, 14.2, 13.5; HRMS (ESI): $\mathrm{m} / \mathrm{z}$ calcd. for $\left[\mathrm{C}_{25} \mathrm{H}_{23} \mathrm{NNaO}_{5}, \mathrm{M}+\mathrm{Na}\right]^{+}$: 440.1468; found: 440.1471 . 
Optical Rotation: $[\alpha]^{25}=+14.2(\mathrm{c}=0.3$, THF). The absolute configuration of 3c was assigned by analogy to 3a. 91\% ee (HPLC condition: Chiralpak IB N-5 column, $n$-hexane $/ i-\operatorname{PrOH}=90: 10$, flow rate $=1 \mathrm{ml} / \mathrm{min}$, wavelength $=254 \mathrm{~nm}, \mathrm{t}_{\mathrm{R}}=12.2 \mathrm{~min}$ for minor isomer, $t_{R}=16.1 \mathrm{~min}$ for major isomer).

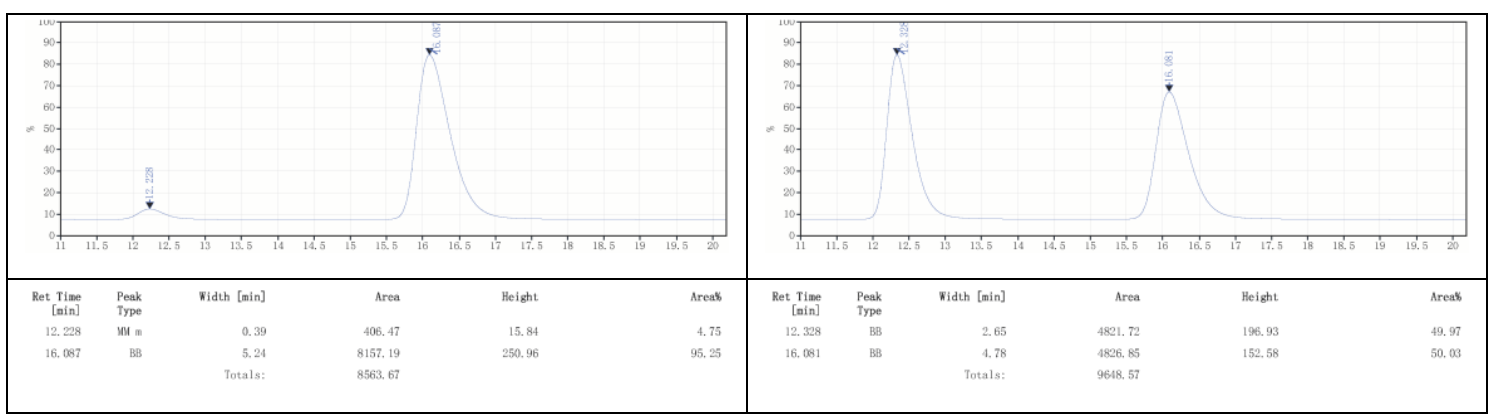

\section{Ethyl}

(S)-5-(1-(3-chloro-6-hydroxy-2,4-dimethylphenyl)naphthalen-2-yl)oxazole-4-carb oxylate (3d)<smiles>CCOC(=O)c1ncoc1-c1ccc2ccccc2c1-c1c(O)cc([N+](=O)[O-])c(Cl)c1O</smiles>

$3 d$

The general procedure outlined above was followed $(0.10 \mathrm{mmol}$ of $\mathbf{2 a}, 5.0 \mathrm{~mL}$ of THF at $15{ }^{\circ} \mathrm{C}$ for $24 \mathrm{~h}$ ). The crude reaction mixture was purified by flash column chromatography (PE/EtOAc 2:1). White solid, $40.1 \mathrm{mg}$, 95\% yield. MP: $172-174{ }^{\circ} \mathrm{C}$; ${ }^{1} \mathbf{H}$ NMR $\left(500 \mathrm{MHz}, \mathrm{CDCl}_{3}\right): \delta 8.01(\mathrm{~d}, J=8.5 \mathrm{~Hz}, 1 \mathrm{H}), 7.96(\mathrm{~d}, J=8.2 \mathrm{~Hz}, 1 \mathrm{H})$, $7.70(\mathrm{~s}, 1 \mathrm{H}), 7.64-7.53(\mathrm{~m}, 2 \mathrm{H}), 7.51-7.36(\mathrm{~m}, 2 \mathrm{H}), 6.69$ (s, 1H), 5.07 (br s, 1H), 4.27 $(\mathrm{q}, J=7.1 \mathrm{~Hz}, 2 \mathrm{H}), 2.35(\mathrm{~s}, 3 \mathrm{H}), 1.92(\mathrm{~s}, 3 \mathrm{H}), 1.25(\mathrm{t}, J=7.1 \mathrm{~Hz}, 3 \mathrm{H}) ;{ }^{13} \mathbf{C}$ NMR $(126$ $\left.\mathrm{MHz}, \mathrm{CDCl}_{3}\right): \delta 161.9,155.9,151.7,150.2,137.6,136.1,134.6,132.3,128.9,128.8$, 128.5, 128.0, 127.7, 127.6, 126.5, 126.2, 126.1, 122.6, 116.0, 61.6, 21.1, 18.3, 14.1; HRMS (ESI): $\mathrm{m} / \mathrm{z}$ calcd. for $\left[\mathrm{C}_{24} \mathrm{H}_{20} \mathrm{ClNNaO}_{4}, \mathrm{M}+\mathrm{Na}\right]^{+}$: 444.0973 ; found: 444.0975 .

Optical Rotation: $[\alpha]^{25}=+52.0(\mathrm{c}=0.3$, THF). The absolute configuration of $\mathbf{3 d}$ was assigned by analogy to 3a. $86 \%$ ee (HPLC condition: Chiralpak IB N-5 column, $n$-hexane $/ i-\mathrm{PrOH}=90: 10$, flow rate $=1 \mathrm{ml} / \mathrm{min}$, wavelength $=254 \mathrm{~nm}, \mathrm{t}_{\mathrm{R}}=10.9 \mathrm{~min}$ for minor isomer, $t_{R}=16.0 \mathrm{~min}$ for major isomer). 


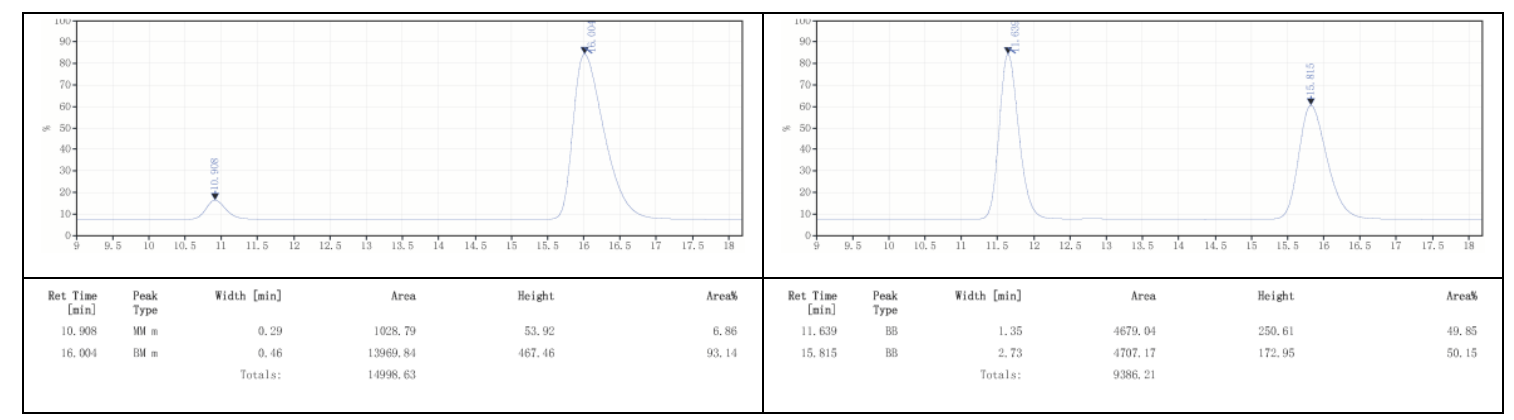

\section{Ethyl}

(R)-5-(1-(2-hydroxy-4,6-dimethoxyphenyl)naphthalen-2-yl)oxazole-4-carboxylate $(3 \mathbf{e})$<smiles>CCOC(=O)c1ncoc1-c1ccc2ccccc2c1-c1c(O)cc(OC)cc1OC</smiles>

The general procedure outlined above was followed $(0.10 \mathrm{mmol}$ of $\mathbf{2 a}, 1.0 \mathrm{~mL}$ of THF at $-20{ }^{\circ} \mathrm{C}$ for $48 \mathrm{~h}$ ). The crude reaction mixture was purified by flash column chromatography (PE/EtOAc 2:1). White solid, $37.7 \mathrm{mg}$, 90\% yield. MP: 186-188 ${ }^{\circ} \mathrm{C}$; ${ }^{1} \mathbf{H}$ NMR $\left(500 \mathrm{MHz}, \mathrm{CDCl}_{3}\right): \delta 7.98(\mathrm{~d}, J=8.4 \mathrm{~Hz}, 1 \mathrm{H}), 7.93(\mathrm{~d}, J=8.2 \mathrm{~Hz}, 1 \mathrm{H})$, $7.72(\mathrm{~s}, 1 \mathrm{H}), 7.66-7.52(\mathrm{~m}, 3 \mathrm{H}), 7.48-7.45(\mathrm{~m}, 1 \mathrm{H}), 6.14(\mathrm{~d}, J=2.3 \mathrm{~Hz}, 1 \mathrm{H}), 6.08(\mathrm{~d}$, $J=2.3 \mathrm{~Hz}, 1 \mathrm{H}), 5.21(\mathrm{br} \mathrm{s}, 1 \mathrm{H}), 4.26(\mathrm{q}, J=7.1 \mathrm{~Hz}, 2 \mathrm{H}), 3.78(\mathrm{~s}, 3 \mathrm{H}), 3.52(\mathrm{~s}, 3 \mathrm{H})$, $1.26(\mathrm{t}, J=7.1 \mathrm{~Hz}, 3 \mathrm{H}) ;{ }^{13} \mathrm{C}$ NMR $\left(126 \mathrm{MHz}, \mathrm{CDCl}_{3}\right): \delta 162.0,161.6,158.8,156.5$, 155.2, 150.0, 134.6, 132.9, 132.2, 128.6, 128.4, 128.3, 127.6, 127.4, 127.2, 127.1, 126.6, 105.7, 93.6, 91.6, 61.4, 55.7, 55.4, 14.2; HRMS (ESI): m/z calcd. for $\left[\mathrm{C}_{24} \mathrm{H}_{21} \mathrm{NNaO}_{6}, \mathrm{M}+\mathrm{Na}\right]^{+}$: 442.1261; found: 442.1262 .

Optical Rotation: $[\alpha]^{25}=-21.2(\mathrm{c}=0.3$, THF). The absolute configuration of $\mathbf{3 e}$ was assigned by analogy to 3a. 91\% ee (HPLC condition: Chiralpak IB N-5 column, $n$-hexane $/ i-\operatorname{PrOH}=90: 10$, flow rate $=1 \mathrm{ml} / \mathrm{min}$, wavelength $=254 \mathrm{~nm}, \mathrm{t}_{\mathrm{R}}=17.9 \mathrm{~min}$ for minor isomer, $\mathrm{t}_{\mathrm{R}}=21.8 \mathrm{~min}$ for major isomer). 


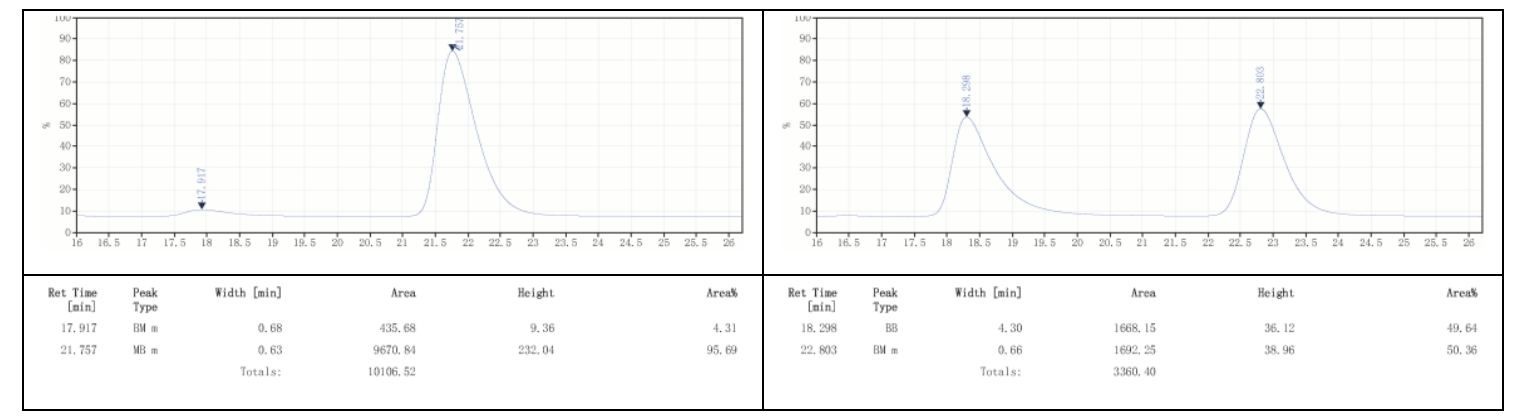

\section{Ethyl}

(R)-5-(1-(6-hydroxy-2,3,4-trimethoxyphenyl)naphthalen-2-yl)oxazole-4-carboxyl ate (3f)<smiles>CCOC(=O)c1ncoc1-c1ccc2ccccc2c1-c1c(O)cc(OC)c(OC)c1OC</smiles>

The general procedure outlined above was followed $(0.10 \mathrm{mmol}$ of $\mathbf{2 a}, 1.0 \mathrm{~mL}$ of $\mathrm{THF}$ at $-20{ }^{\circ} \mathrm{C}$ for $24 \mathrm{~h}$ ). The crude reaction mixture was purified by flash column chromatography (PE/EtOAc 2:1). White syrup, $41.3 \mathrm{mg}, 92 \%$ yield. ${ }^{\mathbf{1}} \mathbf{H}$ NMR (400 MHz, DMSO): $\delta 9.07$ (s, 1H), $8.30(\mathrm{~s}, 1 \mathrm{H}), 8.01$ (d, $J=8.2 \mathrm{~Hz}, 1 \mathrm{H}), 7.98$ (d, $J=8.6$ $\mathrm{Hz}, 1 \mathrm{H}), 7.69(\mathrm{~d}, J=8.5 \mathrm{~Hz}, 1 \mathrm{H}), 7.61-7.55(\mathrm{~m}, 1 \mathrm{H}), 7.54-7.45(\mathrm{~m}, 2 \mathrm{H}), 6.30(\mathrm{~s}, 1 \mathrm{H})$, 4.18-4.05 (m, 2H), 3.76 (s, 3H), 3.57 (s, 3H), 3.26 (s, 3H), 1.08 (t, J = 7.1 Hz, 3H); ${ }^{13}$ C NMR (126 MHz, DMSO): $\delta$ 161.0, 155.7, 153.1, 151.6, 151.4, 150.9, 134.2, 134.0, 133.6, 132.4, 128.1, 127.9, 127.6, 126.9, 126.7, 126.4, 126.3, 125.7, 109.7, 95.2, 60.5, 60.2, 60.0, 55.4, 13.9; HRMS (ESI): m/z calcd. for $\left[\mathrm{C}_{25} \mathrm{H}_{23} \mathrm{NNaO}_{7}\right.$, $\mathrm{M}+\mathrm{Na}]^{+}:$472.1367; found: 472.1370 .

Optical Rotation: $[\alpha]^{25}=-57.1(\mathrm{c}=0.1$, THF). The absolute configuration of $\mathbf{3 f}$ was assigned by analogy to 3a. 91\% ee (HPLC condition: Chiralpak IB N-5 column, $n$-hexane $/ i-\operatorname{PrOH}=90: 10$, flow rate $=1 \mathrm{ml} / \mathrm{min}$, wavelength $=254 \mathrm{~nm}, \mathrm{t}_{\mathrm{R}}=19.3 \mathrm{~min}$ for minor isomer, $t_{R}=29.6 \mathrm{~min}$ for major isomer). 


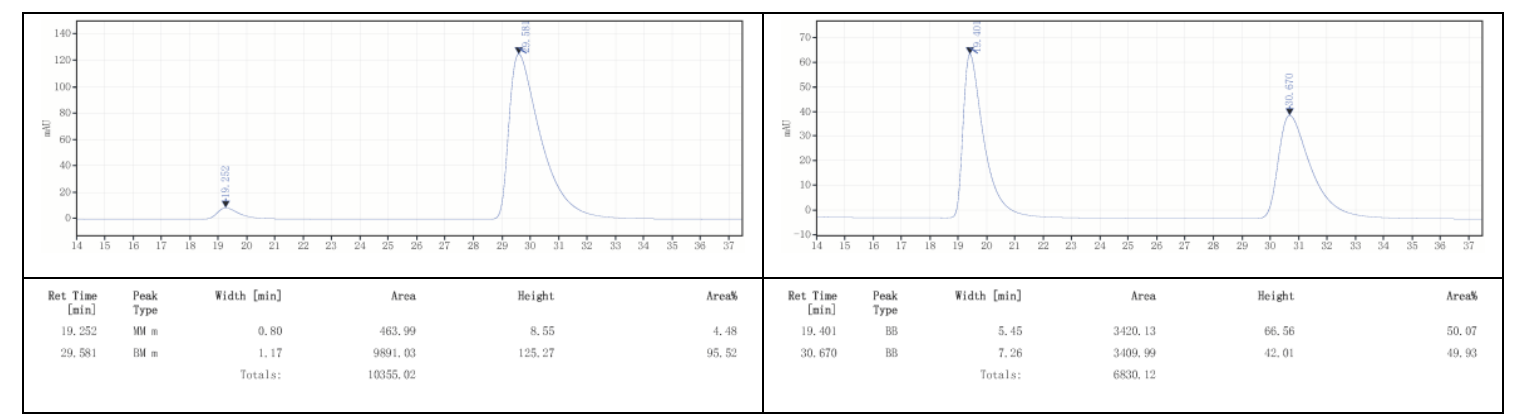

\section{Ethyl}

(S)-5-(2-(2-hydroxynaphthalen-1-yl)-3-methylphenyl)oxazole-4-carboxylate (3g)<smiles>CCOC(=O)c1ncoc1-c1cccc(C)c1-c1c(O)ccc2ccccc12</smiles>

$3 g$

The general procedure outlined above was followed $(0.10 \mathrm{mmol}$ of $\mathbf{2 a}, 5.0 \mathrm{~mL}$ of $\mathrm{THF}$ at $-20{ }^{\circ} \mathrm{C}$ for $48 \mathrm{~h}$ ). The crude reaction mixture was purified by flash column chromatography (PE/EtOAc 2:1). White solid, $33.6 \mathrm{mg}, 90 \%$ yield. MP: $108-110{ }^{\circ} \mathrm{C}$; ${ }^{1} \mathbf{H}$ NMR (500 MHz, $\left.\mathrm{CDCl}_{3}\right): \delta$ 7.76-7.70 (m, 1H), $7.69(\mathrm{~d}, J=8.8 \mathrm{~Hz}, 1 \mathrm{H}), 7.58-7.44$ (m, 3H), 7.36 (s, 1H), 7.30-7.24 (m, 2H), 7.17-7.13 (m, 1H), $7.11(\mathrm{~d}, J=8.9 \mathrm{~Hz}, 1 \mathrm{H})$, 5.59 (br s, 1H), 4.35-4.21 (m, 2H), 1.99 (s, 3H), 1.28 (t, $J=7.1 \mathrm{~Hz}, 3 \mathrm{H}) ;{ }^{13}$ C NMR (126 MHz, $\left.\mathrm{CDCl}_{3}\right): \delta 162.0,156.0,150.7,149.7,140.2,134.4,133.1,132.9,129.9$, 129.5, 129.4, 128.8, 128.6, 128.4, 128.1, 126.5, 124.2, 123.4, 118.0, 117.9, 61.5, 19.9, 14.2; HRMS (ESI): $\mathrm{m} / \mathrm{z}$ calcd. for $\left[\mathrm{C}_{23} \mathrm{H}_{19} \mathrm{NNaO}_{4}, \mathrm{M}+\mathrm{Na}\right]^{+}$: 396.1206; found: 396.1208 .

Optical Rotation: $[\alpha]^{25}=-44.4(\mathrm{c}=0.3$, THF). The absolute configuration of $\mathbf{3 g}$ was assigned by analogy to 3a. 90\% ee (HPLC condition: Chiralpak IB N-5 column, $n$-hexane $/ i-\mathrm{PrOH}=90: 10$, flow rate $=1 \mathrm{ml} / \mathrm{min}$, wavelength $=254 \mathrm{~nm}, \mathrm{t}_{\mathrm{R}}=10.1 \mathrm{~min}$ for minor isomer, $t_{R}=14.2$ min for major isomer).

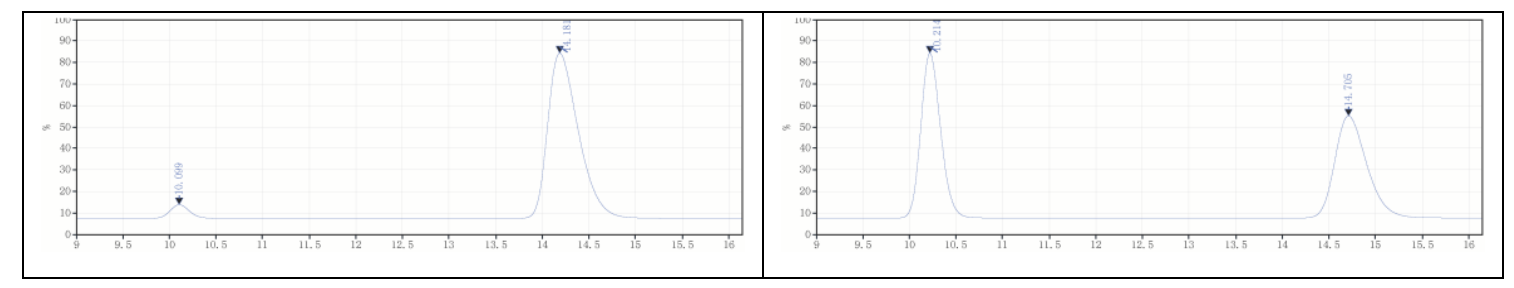




\begin{tabular}{|c|c|c|c|c|c|c|c|c|c|c|c|}
\hline $\begin{array}{l}\text { Ret Time } \\
\text { [rin] }\end{array}$ & $\begin{array}{l}\text { Peak } \\
\text { Type }\end{array}$ & Width [min] & Area & Height & Areas & $\begin{array}{c}\text { Ret Time } \\
\text { [rin] }\end{array}$ & $\begin{array}{l}\text { Peakk } \\
\text { Type }\end{array}$ & Width [min] & Area & Height & Areas \\
\hline 10.099 & Bs & 1. 41 & 600.13 & 41.81 & 4,90 & 10.214 & BB & 1.64 & 2649.90 & 179.06 & 49.87 \\
\hline 14. 181 & $\mathrm{BB}$ & $\begin{array}{r}3.06 \\
\text { Totals: }\end{array}$ & $\begin{array}{l}11656.55 \\
12556.68\end{array}$ & 509.13 & 95,10 & 14.705 & BBA & $\begin{array}{r}3.99 \\
\text { Totalas: }\end{array}$ & $\begin{array}{l}2663.74 \\
5313.64\end{array}$ & 110.88 & 50.13 \\
\hline
\end{tabular}

Ethyl (S)-5-(3-ethyl-2-(2-hydroxynaphthalen-1-yl)phenyl)oxazole-4-carboxylate (3h)<smiles>CCOC(=O)c1ncoc1-c1cccc(O)c1-c1c(CC)ccc2ccccc12</smiles>

$3 \mathrm{~h}$

The general procedure outlined above was followed $(0.10 \mathrm{mmol}$ of $\mathbf{2 a}, 5.0 \mathrm{~mL}$ of $\mathrm{THF}$ at $-10{ }^{\circ} \mathrm{C}$ for $24 \mathrm{~h}$ ). The crude reaction mixture was purified by flash column chromatography (PE/EtOAc 2:1). Colourless wax, $34.1 \mathrm{mg}, 88 \%$ yield. ${ }^{1} \mathbf{H}$ NMR (500 $\left.\mathrm{MHz}, \mathrm{CDCl}_{3}\right): \delta 7.75-7.66(\mathrm{~m}, 2 \mathrm{H}), 7.63(\mathrm{dd}, J=7.8,1.3 \mathrm{~Hz}, 1 \mathrm{H}), 7.59(\mathrm{t}, J=7.7 \mathrm{~Hz}$, 1H), 7.48 (dd, $J=7.5,1.4 \mathrm{~Hz}, 1 \mathrm{H}), 7.35(\mathrm{~s}, 1 \mathrm{H}), 7.30-7.26(\mathrm{~m}, 2 \mathrm{H}), 7.18-7.14(\mathrm{~m}$, $1 \mathrm{H}), 7.10(\mathrm{~d}, J=8.9 \mathrm{~Hz}, 1 \mathrm{H}), 4.38-4.20(\mathrm{~m}, 2 \mathrm{H}), 2.30(\mathrm{q}, J=7.6 \mathrm{~Hz}, 2 \mathrm{H}), 1.29(\mathrm{t}, J=$ $7.1 \mathrm{~Hz}, 3 \mathrm{H}), 0.98(\mathrm{t}, J=7.6 \mathrm{~Hz}, 3 \mathrm{H}) ;{ }^{13} \mathbf{C ~ N M R}\left(126 \mathrm{MHz}, \mathrm{CDCl}_{3}\right): \delta$ 162.1, 156.1, $151.0,149.7,146.1,133.7,133.4,131.6,130.0,129.7,129.6,129.0,128.8,128.6$, 128.0, 126.5, 124.6, 123.5, 117.9, 117.6, 61.5, 26.4, 14.9, 14.2; HRMS (ESI): m/z calcd. for $\left[\mathrm{C}_{24} \mathrm{H}_{21} \mathrm{NNaO}_{4}, \mathrm{M}+\mathrm{Na}\right]^{+}:$: 10.1363 ; found: 410.1365 .

Optical Rotation: $[\alpha]^{25}=-57.0(\mathrm{c}=0.1, \mathrm{THF})$. The absolute configuration of $\mathbf{3 h}$ was assigned by analogy to 3a. $87 \%$ ee (HPLC condition: Chiralpak IB N-5 column, $n$-hexane $/ i-\operatorname{PrOH}=90: 10$, flow rate $=1 \mathrm{ml} / \mathrm{min}$, wavelength $=254 \mathrm{~nm}, \mathrm{t}_{\mathrm{R}}=8.9 \mathrm{~min}$ for minor isomer, $t_{R}=11.2$ min for major isomer).

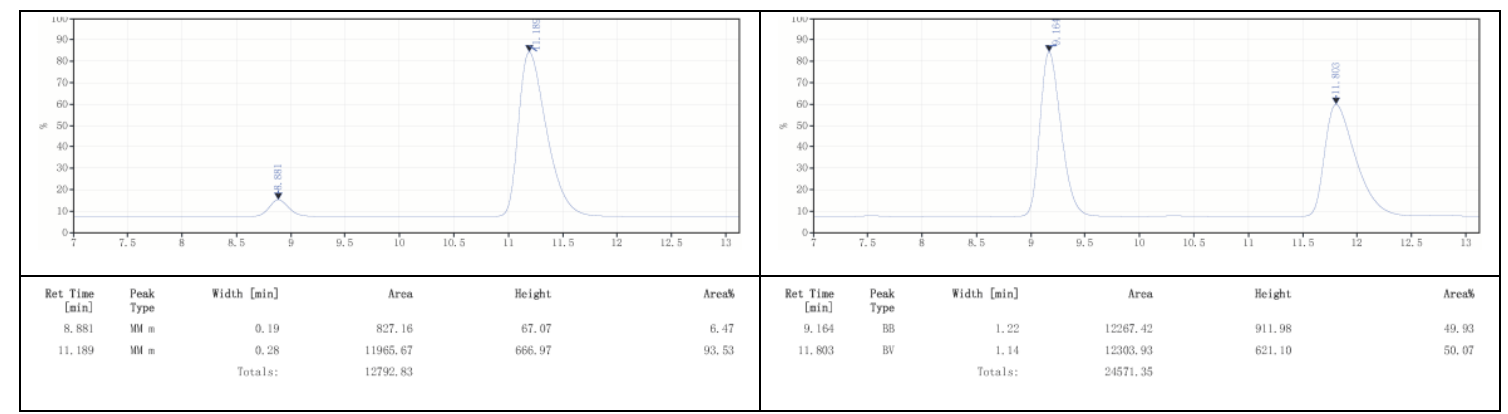

Ethyl

(S)-5-(2-(2-hydroxynaphthalen-1-yl)-3,5-dimethylphenyl)oxazole-4-carboxylate 
(3i)<smiles>CCOC(=O)c1ncoc1-c1cc(C)cc(C)c1-c1c(O)ccc2ccccc12</smiles>

$3 \mathbf{i}$

The general procedure outlined above was followed $(0.10 \mathrm{mmol}$ of $\mathbf{2 a}, 5.0 \mathrm{~mL}$ of THF at $-20{ }^{\circ} \mathrm{C}$ for $24 \mathrm{~h}$ ). The crude reaction mixture was purified by flash column chromatography (PE/EtOAc 2:1). White solid, $36.8 \mathrm{mg}, 95 \%$ yield. MP: $131-133{ }^{\circ} \mathrm{C}$; ${ }^{1} \mathbf{H}$ NMR (500 MHz, $\left.\mathrm{CDCl}_{3}\right): \delta$ 7.73-7.69 (m, 1H), $7.67(\mathrm{~d}, J=8.9 \mathrm{~Hz}, 1 \mathrm{H}), 7.40-7.37$ (m, 1H), $7.36(\mathrm{~s}, 1 \mathrm{H}), 7.31-7.24(\mathrm{~m}, 3 \mathrm{H}), 7.21-7.13(\mathrm{~m}, 1 \mathrm{H}), 7.10(\mathrm{~d}, J=8.9 \mathrm{~Hz}, 1 \mathrm{H})$, 5.52 (br s, 1H), 4.36-4.16 (m, 2H), 2.46 (s, 3H), 1.94 (s, 3H), 1.29 (t, J = 7.1 Hz, 3H); ${ }^{13} \mathrm{C}$ NMR (126 MHz, $\left.\mathrm{CDCl}_{3}\right): \delta 162.0,156.2,150.8,149.6,139.9,138.5,134.0$, $133.1,131.2,129.9,129.8,129.4,128.8,128.3,128.0,126.4,124.3,123.4,117.9$, 117.8, 61.4, 21.2, 19.8, 14.2; HRMS (ESI): $\mathrm{m} / \mathrm{z}$ calcd. for $\left[\mathrm{C}_{24} \mathrm{H}_{21} \mathrm{NNaO}_{4}, \mathrm{M}+\mathrm{Na}\right]^{+}$: 410.1363; found: 410.1362 .

Optical Rotation: $[\alpha]^{25}=-74.6(\mathrm{c}=0.3$, THF $)$. The absolute configuration of $\mathbf{3 i}$ was assigned by analogy to 3a. 90\% ee (HPLC condition: Chiralpak IB N-5 column, $n$-hexane $/ i-\operatorname{PrOH}=90: 10$, flow rate $=1 \mathrm{ml} / \mathrm{min}$, wavelength $=254 \mathrm{~nm}, \mathrm{t}_{\mathrm{R}}=14.1 \mathrm{~min}$ for minor isomer, $t_{R}=14.9 \mathrm{~min}$ for major isomer).

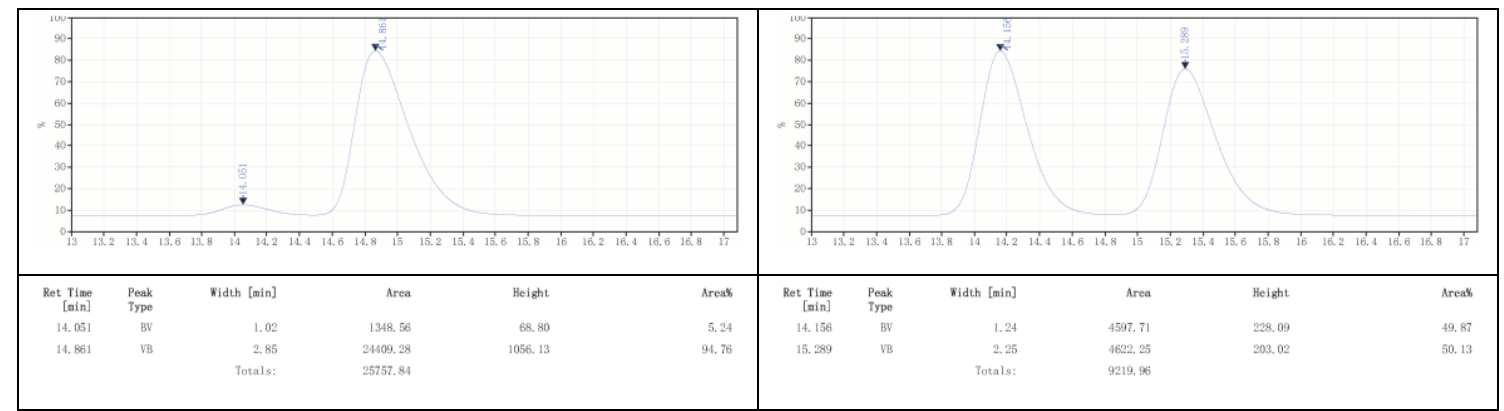

\section{Ethyl}

(S)-5-(2-(6-bromo-2-hydroxynaphthalen-1-yl)-3-methylphenyl)oxazole-4-carboxy late (3j) 


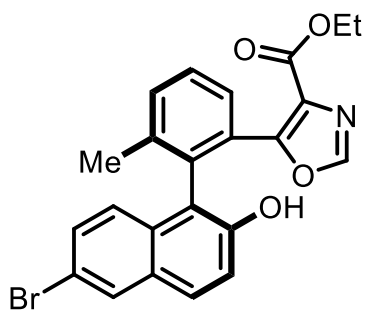

3j

The general procedure outlined above was followed $(0.10 \mathrm{mmol}$ of $\mathbf{2 a}, 5.0 \mathrm{~mL}$ of $\mathrm{THF}$ at $-20^{\circ} \mathrm{C}$ for $48 \mathrm{~h}$ ). The crude reaction mixture was purified by flash column chromatography (PE/EtOAc 2:1). White solid, $40.7 \mathrm{mg}$, 90\% yield. MP: $177-179{ }^{\circ} \mathrm{C}$; ${ }^{1} \mathbf{H}$ NMR (500 MHz, $\left.\mathrm{CDCl}_{3}\right): \delta 7.87(\mathrm{~d}, J=2.0 \mathrm{~Hz}, 1 \mathrm{H}), 7.59(\mathrm{~d}, J=8.9 \mathrm{~Hz}, 1 \mathrm{H})$, 7.57-7.45 (m, 3H), $7.40(\mathrm{~s}, 1 \mathrm{H}), 7.34(\mathrm{dd}, J=9.0,2.0 \mathrm{~Hz}, 1 \mathrm{H}), 7.12(\mathrm{~d}, J=8.9 \mathrm{~Hz}$, $1 \mathrm{H}), 7.03(\mathrm{~d}, J=9.0 \mathrm{~Hz}, 1 \mathrm{H}), 5.61(\mathrm{br} \mathrm{s}, 1 \mathrm{H}), 4.34-4.09(\mathrm{~m}, 2 \mathrm{H}), 1.97(\mathrm{~s}, 3 \mathrm{H}), 1.29$ (t, $J=7.1 \mathrm{~Hz}, 3 \mathrm{H}) ;{ }^{13} \mathbf{C}$ NMR $\left(126 \mathrm{MHz}, \mathrm{CDCl}_{3}\right): \delta 162.0,155.8,151.1,149.8,140.1$, $133.8,133.2$, 131.5, 130.1, 129.9, 129.8, 129.5, 129.5, 129.1, 128.9, 128.5, 126.1, 119.3, 118.1, 117.2, 61.6, 19.8, 14.2; HRMS (ESI): m/z calcd. for $\left[\mathrm{C}_{23} \mathrm{H}_{18} \mathrm{BrNNaO}_{4}\right.$, $\mathrm{M}+\mathrm{Na}]^{+}:$474.0311; found: 474.0313 .

Optical Rotation: $[\alpha]^{25}=-60.9(\mathrm{c}=0.5$, THF). The absolute configuration of $\mathbf{3 j}$ was assigned by analogy to 3a. $86 \%$ ee (HPLC condition: Chiralpak IB N-5 column, $n$-hexane $/ i-\operatorname{PrOH}=90: 10$, flow rate $=1 \mathrm{ml} / \mathrm{min}$, wavelength $=254 \mathrm{~nm}, \mathrm{t}_{\mathrm{R}}=10.7 \mathrm{~min}$ for minor isomer, $\mathrm{t}_{\mathrm{R}}=15.8 \mathrm{~min}$ for major isomer).

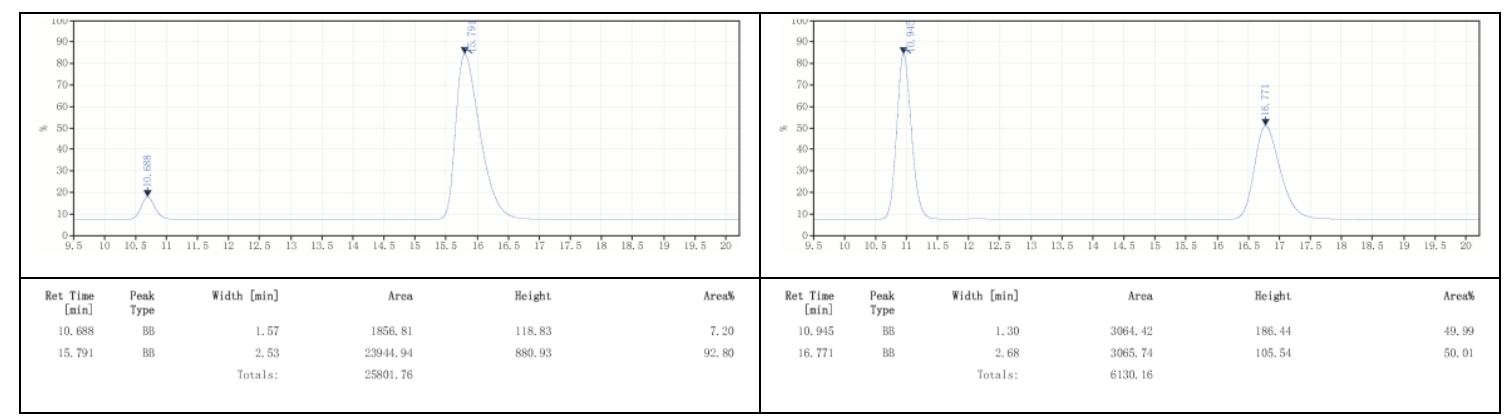

\section{Ethyl}

(S)-5-(2-(6-bromo-2-hydroxynaphthalen-1-yl)-3,5-dimethylphenyl)oxazole-4-carb oxylate (3k) 


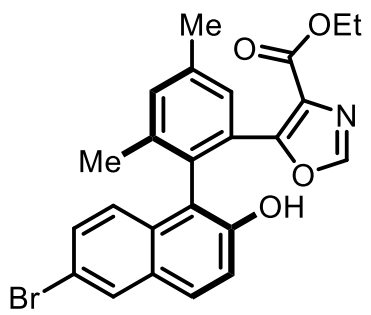

3k

The general procedure outlined above was followed $(0.10 \mathrm{mmol}$ of $\mathbf{2 a}, 5.0 \mathrm{~mL}$ of THF at $-20{ }^{\circ} \mathrm{C}$ for $48 \mathrm{~h}$ ). The crude reaction mixture was purified by flash column chromatography (PE/EtOAc 2:1). White solid, $41.5 \mathrm{mg}, 89 \%$ yield. MP: 176-178 ${ }^{\circ} \mathrm{C}$; ${ }^{1} \mathbf{H}$ NMR $\left(500 \mathrm{MHz}, \mathrm{CDCl}_{3}\right): \delta 7.86(\mathrm{~d}, J=1.9 \mathrm{~Hz}, 1 \mathrm{H}), 7.57(\mathrm{~d}, J=8.9 \mathrm{~Hz}, 1 \mathrm{H})$, $7.38(\mathrm{~s}, 1 \mathrm{H}), 7.36(\mathrm{~s}, 1 \mathrm{H}), 7.32(\mathrm{dd}, J=9.0,2.0 \mathrm{~Hz}, 1 \mathrm{H}), 7.28(\mathrm{~s}, 1 \mathrm{H}), 7.11(\mathrm{~d}, J=8.9$ $\mathrm{Hz}, 1 \mathrm{H}), 7.04(\mathrm{~d}, J=9.0 \mathrm{~Hz}, 1 \mathrm{H}), 5.66(\mathrm{br} \mathrm{s}, 1 \mathrm{H}), 4.47-3.96(\mathrm{~m}, 2 \mathrm{H}), 2.45(\mathrm{~s}, 3 \mathrm{H})$, $1.92(\mathrm{~s}, 3 \mathrm{H}), 1.29$ (t, $J=7.1 \mathrm{~Hz}, 3 \mathrm{H}) ;{ }^{13} \mathrm{C} \mathrm{NMR}\left(126 \mathrm{MHz}, \mathrm{CDCl}_{3}\right): \delta$ 162.0, 156.0, $151.2,149.7,139.8,138.8,134.1,131.7,130.6,130.0,129.9,129.7,129.3,128.9$, 128.3, 126.2, 119.2, 118.1, 117.1, 61.5, 21.2, 19.7, 14.2; HRMS (ESI): m/z calcd. for $\left[\mathrm{C}_{24} \mathrm{H}_{20} \mathrm{BrNNaO}_{4}, \mathrm{M}+\mathrm{Na}\right]^{+}$: 488.0468; found: 488.0470 .

Optical Rotation: $[\alpha]^{25}=-61.4(\mathrm{c}=0.5$, THF). The absolute configuration of $\mathbf{3 k}$ was assigned by analogy to 3a. $86 \%$ ee (HPLC condition: Chiralpak IB N-5 column, $n$-hexane $/ i-\operatorname{PrOH}=90: 10$, flow rate $=1 \mathrm{ml} / \mathrm{min}$, wavelength $=254 \mathrm{~nm}, \mathrm{t}_{\mathrm{R}}=9.7 \mathrm{~min}$ for minor isomer, $t_{R}=10.7 \mathrm{~min}$ for major isomer).

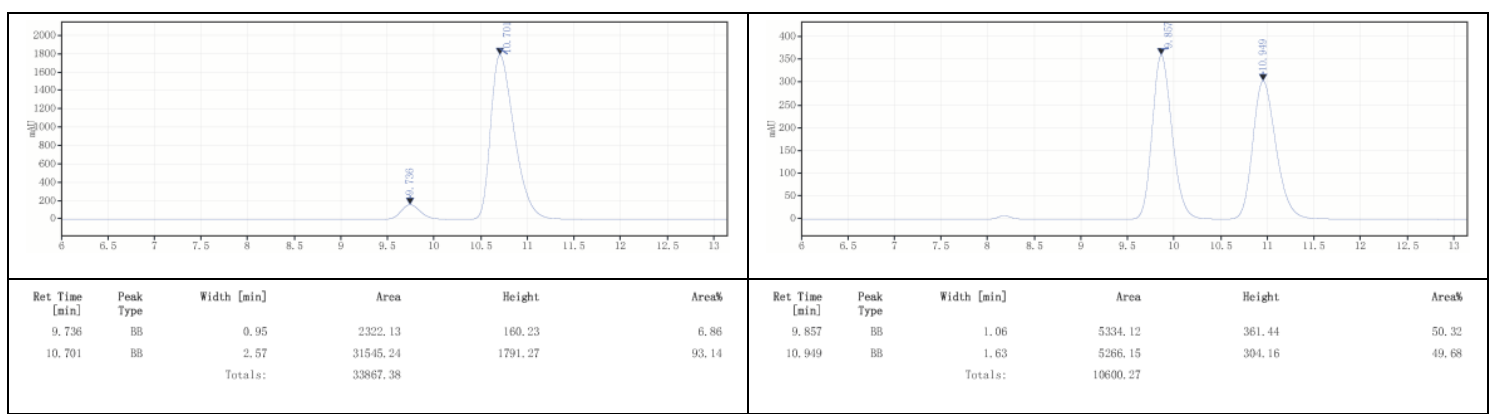

\section{Ethyl}

(S)-5-(2-(7-bromo-2-hydroxynaphthalen-1-yl)-3-methylphenyl)oxazole-4-carboxy late (3I) 
<smiles>CCOC(=O)c1ncoc1-c1cccc(C)c1-c1c(O)ccc2ccc(Br)cc12</smiles>

3I

The general procedure outlined above was followed $(0.10 \mathrm{mmol}$ of $\mathbf{2 a}, 5.0 \mathrm{~mL}$ of THF at $-20{ }^{\circ} \mathrm{C}$ for $48 \mathrm{~h}$ ). The crude reaction mixture was purified by flash column chromatography (PE/EtOAc 2:1). White solid, $40.7 \mathrm{mg}$, $90 \%$ yield. MP: $96-98{ }^{\circ} \mathrm{C} ;{ }^{1} \mathbf{H}$ NMR (500 MHz, $\left.\mathrm{CDCl}_{3}\right): \delta 7.64(\mathrm{~d}, J=8.9 \mathrm{~Hz}, 1 \mathrm{H}), 7.58-7.55(\mathrm{~m}, 2 \mathrm{H}), 7.54-7.45(\mathrm{~m}$, 2H), $7.40(\mathrm{~s}, 1 \mathrm{H}), 7.34(\mathrm{dd}, J=8.6,1.9 \mathrm{~Hz}, 1 \mathrm{H}), 7.28(\mathrm{~d}, J=1.9 \mathrm{~Hz}, 1 \mathrm{H}), 7.12(\mathrm{~d}, J=$ $8.9 \mathrm{~Hz}, 1 \mathrm{H}), 5.68$ (br s, 1H), 4.41-4.14 (m, 2H), 1.99 (s, 3H), 1.28 (t, J=7.1 Hz, 3H); ${ }^{13} \mathrm{C}$ NMR (126 MHz, $\left.\mathrm{CDCl}_{3}\right): \delta 161.8,155.7,151.7,149.8,140.0,134.1,133.5$, $133.3,129.9,129.8,129.7,129.5,129.0,128.5,127.2,126.9,126.2,121.2,118.5$, 117.2, 61.6, 19.9, 14.2; HRMS (ESI): $\mathrm{m} / \mathrm{z}$ calcd. for $\left[\mathrm{C}_{23} \mathrm{H}_{18} \mathrm{BrNNaO}_{4}, \mathrm{M}+\mathrm{Na}\right]^{+}$: 474.0311; found: 474.0312 .

Optical Rotation: $[\alpha]^{25}=-85.3(\mathrm{c}=0.5$, THF). The absolute configuration of $\mathbf{3 1}$ was assigned by analogy to 3a. 90\% ee (HPLC condition: Chiralpak IB N-5 column, $n$-hexane $/ i-\operatorname{PrOH}=90: 10$, flow rate $=1 \mathrm{ml} / \mathrm{min}$, wavelength $=254 \mathrm{~nm}, \mathrm{t}_{\mathrm{R}}=12.6 \mathrm{~min}$ for minor isomer, $t_{R}=16.3 \mathrm{~min}$ for major isomer).

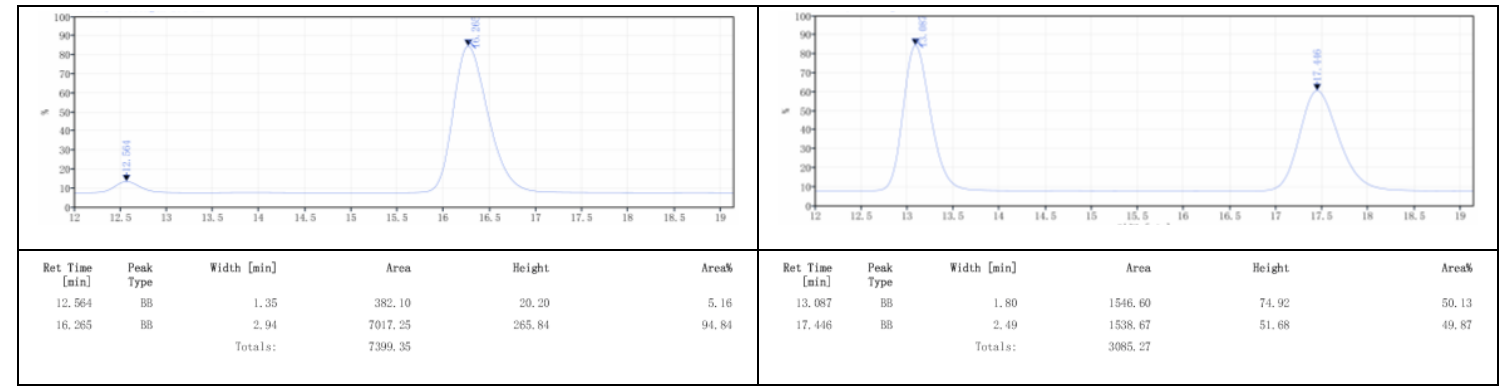

\section{Ethyl}

(S)-5-(2-(2-hydroxy-7-methoxynaphthalen-1-yl)-3-methylphenyl)oxazole-4-carbo xylate $(3 \mathrm{~m})$ 


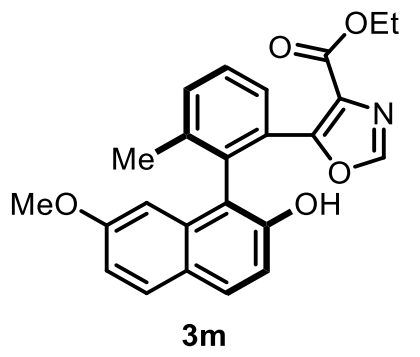

The general procedure outlined above was followed $(0.10 \mathrm{mmol}$ of $\mathbf{2 a}, 5.0 \mathrm{~mL}$ of $\mathrm{THF}$ at $-20{ }^{\circ} \mathrm{C}$ for $48 \mathrm{~h}$ ). The crude reaction mixture was purified by flash column chromatography (PE/EtOAc 2:1). Colourless wax, $34.7 \mathrm{mg}, 86 \%$ yield. ${ }^{1} \mathbf{H}$ NMR (500 $\left.\mathrm{MHz}, \mathrm{CDCl}_{3}\right): \delta 7.66-7.53(\mathrm{~m}, 3 \mathrm{H}), 7.52-7.45(\mathrm{~m}, 2 \mathrm{H}), 7.40(\mathrm{~s}, 1 \mathrm{H}), 6.96(\mathrm{~d}, J=8.8$ $\mathrm{Hz}, 1 \mathrm{H}), 6.92(\mathrm{dd}, J=8.9,2.5 \mathrm{~Hz}, 1 \mathrm{H}), 6.42(\mathrm{~d}, J=2.3 \mathrm{~Hz}, 1 \mathrm{H}), 5.46(\mathrm{~s}, 1 \mathrm{H})$, 4.37-4.16 (m, 2H), $3.67(\mathrm{~s}, 3 \mathrm{H}), 2.01(\mathrm{~s}, 3 \mathrm{H}), 1.28(\mathrm{t}, J=7.1 \mathrm{~Hz}, 3 \mathrm{H}) ;{ }^{13} \mathbf{C}$ NMR (126 $\left.\mathrm{MHz}, \mathrm{CDCl}_{3}\right): \delta 162.0,158.4,156.1,151.4,149.6,140.1,134.5,134.2,133.2,129.7$, 129.6, 129.6, 129.5, 128.7, 128.3, 124.2, 117.2, 115.4, 115.4, 103.3, 61.5, 55.1, 19.8, 14.2; HRMS (ESI): $\mathrm{m} / \mathrm{z}$ calcd. for $\left[\mathrm{C}_{24} \mathrm{H}_{21} \mathrm{NNaO}_{5}, \mathrm{M}+\mathrm{Na}\right]^{+}$: 426.1312; found: 426.1313 .

Optical Rotation: $[\alpha]^{25}=-229.2(\mathrm{c}=0.3$, THF). The absolute configuration of $\mathbf{3 m}$ was assigned by analogy to 3a. 90\% ee (HPLC condition: Chiralpak IB N-5 column, $n$-hexane $/ i-\mathrm{PrOH}=90: 10$, flow rate $=1 \mathrm{ml} / \mathrm{min}$, wavelength $=254 \mathrm{~nm}, \mathrm{t}_{\mathrm{R}}=11.7 \mathrm{~min}$ for minor isomer, $t_{\mathrm{R}}=13.9 \mathrm{~min}$ for major isomer).

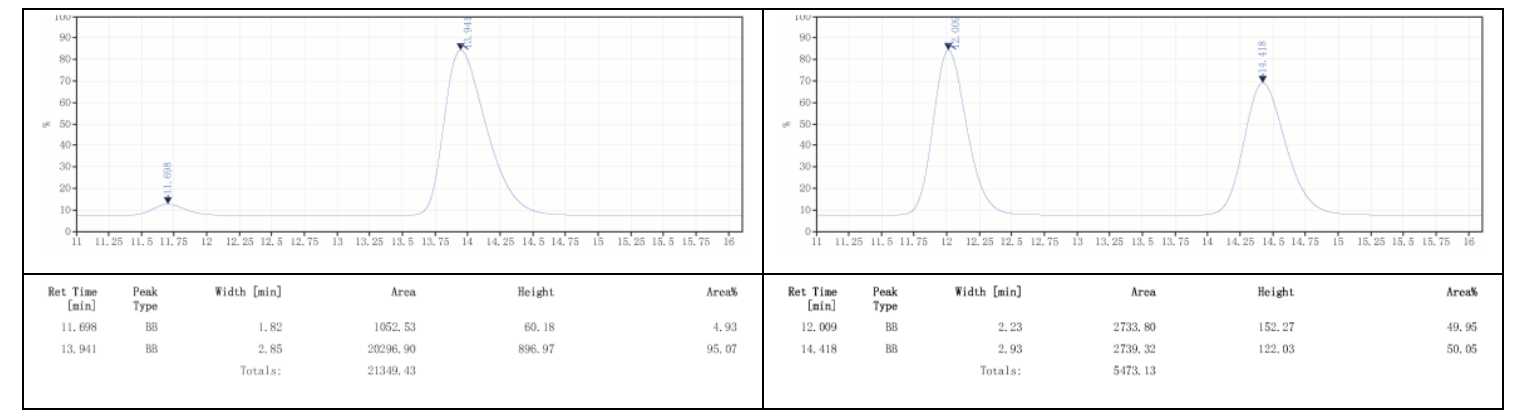

\section{Ethyl}

(S)-5-(2'-hydroxy-4',6,6'-trimethyl-[1,1'-biphenyl]-2-yl)oxazole-4-carboxylate $(3 n)$ 


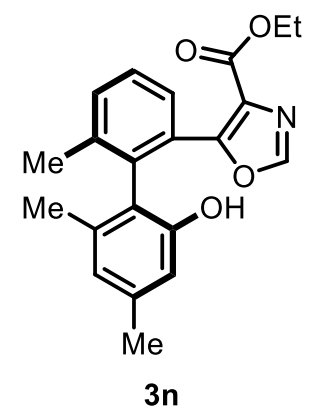

The general procedure outlined above was followed $(0.12 \mathrm{mmol}$ of $\mathbf{2 a}, 5.0 \mathrm{~mL}$ of THF at $0{ }^{\circ} \mathrm{C}$ for $24 \mathrm{~h}$ ). The crude reaction mixture was purified by flash column chromatography (PE/EtOAc 2:1). White solid, $28.4 \mathrm{mg}, 81 \%$ yield. MP: $126-128{ }^{\circ} \mathrm{C}$; ${ }^{1} \mathbf{H}$ NMR (500 MHz, $\left.\mathrm{CDCl}_{3}\right): \delta 7.62(\mathrm{~s}, 1 \mathrm{H}), 7.51-7.46(\mathrm{~m}, 1 \mathrm{H}), 7.44-7.36(\mathrm{~m}, 2 \mathrm{H})$, $6.52(\mathrm{~s}, 1 \mathrm{H}), 6.51(\mathrm{~s}, 1 \mathrm{H}), 4.93(\mathrm{br} \mathrm{s}, 1 \mathrm{H}), 4.38-4.14(\mathrm{~m}, 2 \mathrm{H}), 2.21(\mathrm{~s}, 3 \mathrm{H}), 2.06$ (s, $3 \mathrm{H}), 1.86(\mathrm{~s}, 3 \mathrm{H}), 1.26(\mathrm{t}, J=7.1 \mathrm{~Hz}, 3 \mathrm{H}) ;{ }^{13} \mathbf{C ~ N M R}\left(126 \mathrm{MHz}, \mathrm{CDCl}_{3}\right): \delta$ 162.0, $156.2,152.7,149.8,139.3,138.9,136.9,135.9,132.9,129.4,128.7,128.3,128.2$, 123.0, 122.1, 114.1, 61.5, 21.3, 19.8, 19.7, 14.1; HRMS (ESI): $\mathrm{m} / \mathrm{z}$ calcd. for $\left[\mathrm{C}_{21} \mathrm{H}_{21} \mathrm{NNaO}_{4}, \mathrm{M}+\mathrm{Na}\right]^{+}$: 374.1363; found: 374.1364.

Optical Rotation: $[\alpha]^{25}=+55.4(\mathrm{c}=0.3$, THF $)$. The absolute configuration of $\mathbf{3 n}$ was assigned by analogy to 3a. 90\% ee (HPLC condition: Chiralcel IB N-5 column, $n$-hexane $/ i$-PrOH $=90: 10$, flow rate $=1 \mathrm{ml} / \mathrm{min}$, wavelength $=254 \mathrm{~nm}, \mathrm{t}_{\mathrm{R}}=7.6 \mathrm{~min}$ for minor isomer, $t_{R}=11.0 \mathrm{~min}$ for major isomer).

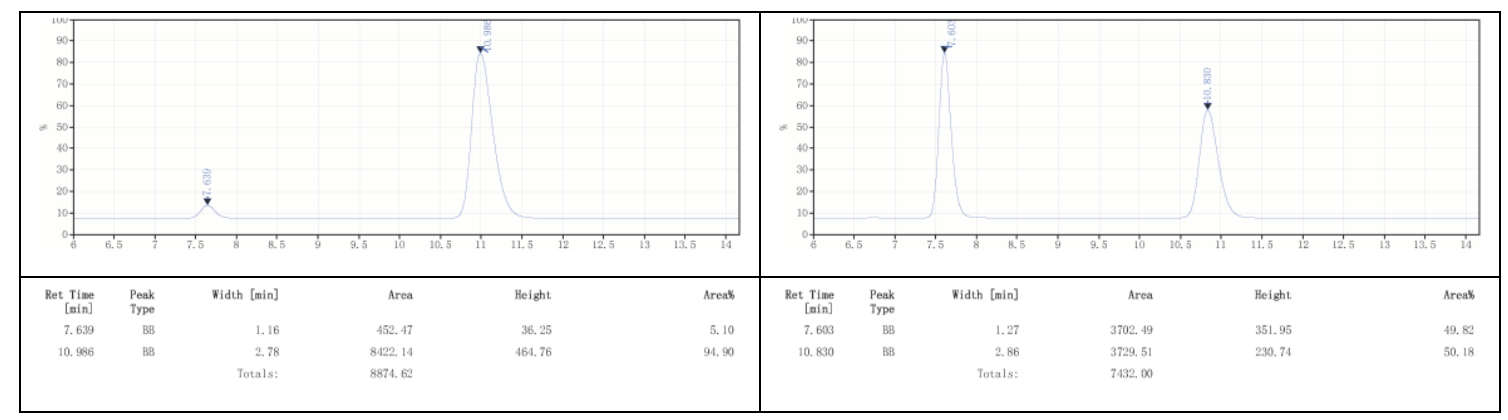

\section{Ethyl}

(S)-5-(6'-hydroxy-2',3',4',6-tetramethyl-[1,1'-biphenyl]-2-yl)oxazole-4-carboxylat e (3o) 
(O)

30

The general procedure outlined above was followed $(0.10 \mathrm{mmol}$ of $\mathbf{2 a}, 1.0 \mathrm{~mL}$ of THF at $15{ }^{\circ} \mathrm{C}$ for $24 \mathrm{~h}$ ). The crude reaction mixture was purified by flash column chromatography (PE/EtOAc 2:1). Yellow wax, $33.6 \mathrm{mg}, 92 \%$ yield. ${ }^{1} \mathbf{H}$ NMR (500 $\mathrm{MHz}, \mathrm{CDCl}_{3}$ ): $\delta 7.61(\mathrm{~s}, 1 \mathrm{H}), 7.49(\mathrm{dd}, J=7.2,0.6 \mathrm{~Hz}, 1 \mathrm{H}), 7.44-7.36(\mathrm{~m}, 2 \mathrm{H}), 6.54$ (s, 1H), 4.71 (br s, 1H), 4.33-4.21 (m, 2H), 2.19 (s, 3H), 2.04 (s, 3H), 2.03 (s, 3H), $1.81(\mathrm{~s}, 3 \mathrm{H}), 1.26(\mathrm{t}, J=7.1 \mathrm{~Hz}, 3 \mathrm{H}) ;{ }^{13} \mathbf{C} \mathbf{N M R}\left(126 \mathrm{MHz}, \mathrm{CDCl}_{3}\right): \delta 161.9,156.3$, $150.1,149.8,139.4,137.3,136.8,134.9,132.8,129.4,128.7,128.3,128.0,127.0$, 122.7, 114.9, 61.4, 20.9, 20.0, 17.2, 15.3, 14.2; HRMS (ESI): m/z calcd. for $\left[\mathrm{C}_{22} \mathrm{H}_{23} \mathrm{NNaO}_{4}, \mathrm{M}+\mathrm{Na}\right]^{+}$: 388.1519; found: 388.1521 .

Optical Rotation: $[\alpha]^{25}=+18.9$ (c $=0.3$, THF). The absolute configuration of 3o was assigned by analogy to 3a. 84\% ee (HPLC condition: Chiralpak IB N-5 column, $n$-hexane $/ i-\operatorname{PrOH}=90: 10$, flow rate $=1 \mathrm{ml} / \mathrm{min}$, wavelength $=254 \mathrm{~nm}, \mathrm{t}_{\mathrm{R}}=8.1 \mathrm{~min}$ for minor isomer, $t_{R}=12.1 \mathrm{~min}$ for major isomer).

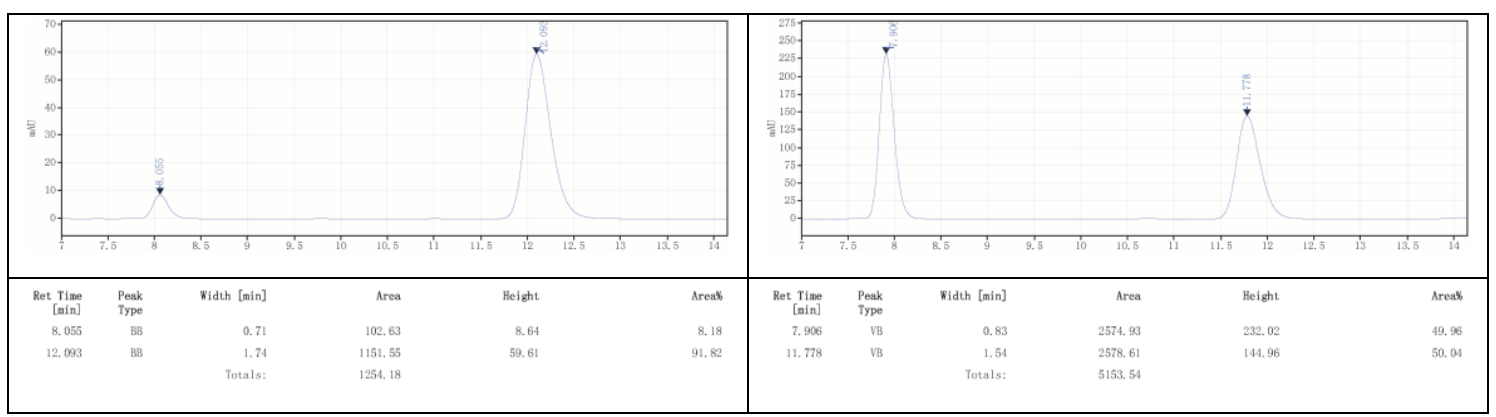

\section{Ethyl}

(S)-5-(6'-hydroxy-3'-methoxy-2',4',6-trimethyl-[1,1'-biphenyl]-2-yl)oxazole-4-car boxylate (3p) 


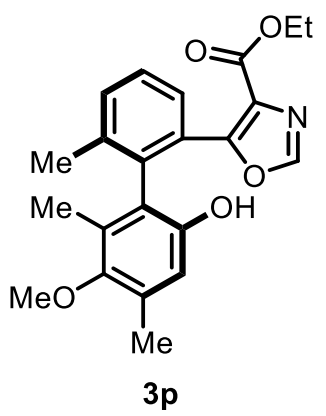

The general procedure outlined above was followed $(0.10 \mathrm{mmol}$ of $\mathbf{2 a}, 5.0 \mathrm{~mL}$ of $\mathrm{THF}$ at $-20{ }^{\circ} \mathrm{C}$ for $72 \mathrm{~h}$ ). The crude reaction mixture was purified by flash column chromatography (PE/EtOAc 2:1). Colourless wax, $30.1 \mathrm{mg}, 79 \%$ yield. ${ }^{1} \mathbf{H}$ NMR (500 $\left.\mathrm{MHz}, \mathrm{CDCl}_{3}\right): \delta 7.62(\mathrm{~s}, 1 \mathrm{H}), 7.49(\mathrm{~d}, J=7.4 \mathrm{~Hz}, 1 \mathrm{H}), 7.44-7.36(\mathrm{~m}, 2 \mathrm{H}), 6.51(\mathrm{~s}$, 1H), 4.34-4.20 (m, 2H), $3.58(\mathrm{~s}, 3 \mathrm{H}), 2.20(\mathrm{~s}, 3 \mathrm{H}), 2.05(\mathrm{~s}, 3 \mathrm{H}), 1.84(\mathrm{~s}, 3 \mathrm{H}), 1.26(\mathrm{t}, J$ $=7.1 \mathrm{~Hz}, 3 \mathrm{H}) ;{ }^{13} \mathbf{C}$ NMR $\left(126 \mathrm{MHz}, \mathrm{CDCl}_{3}\right): \delta 162.0,156.1,150.7,149.8,148.6$, 139.1, 135.9, 132.9, 131.5, 129.6, 129.3, 128.5, 128.4, 128.3, 123.8, 115.8, 61.5, 60.1, 19.8, 16.2, 14.1, 13.3; HRMS (ESI): $\mathrm{m} / \mathrm{z}$ calcd. for $\left[\mathrm{C}_{22} \mathrm{H}_{23} \mathrm{NNaO}_{5}, \mathrm{M}+\mathrm{Na}\right]^{+}$: 404.1468; found: 404.1469 .

Optical Rotation: $[\alpha]^{25}=+30.0(\mathrm{c}=0.1$, THF). The absolute configuration of $\mathbf{3 p}$ was assigned by analogy to 3a. 93\% ee (HPLC condition: Chiralpak IB N-5 column, $n$-hexane $/ i-\operatorname{PrOH}=90: 10$, flow rate $=1 \mathrm{ml} / \mathrm{min}$, wavelength $=254 \mathrm{~nm}, \mathrm{t}_{\mathrm{R}}=8.8 \mathrm{~min}$ for minor isomer, $t_{R}=13.5 \mathrm{~min}$ for major isomer).

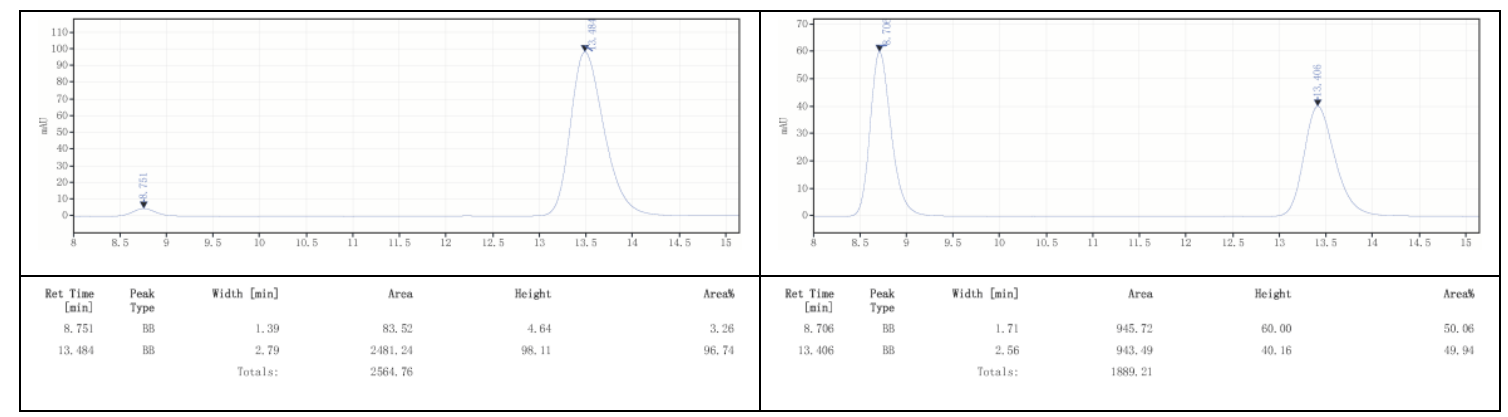

\section{Ethyl}

(S)-5-(3'-chloro-6'-hydroxy-2',4',6-trimethyl-[1,1'-biphenyl]-2-yl)oxazole-4-carbo xylate (3q) 
(1)

$3 q$

The general procedure outlined above was followed $(0.10 \mathrm{mmol}$ of $\mathbf{2 a}, 5.0 \mathrm{~mL}$ of THF at $15{ }^{\circ} \mathrm{C}$ for $24 \mathrm{~h}$ ). The crude reaction mixture was purified by flash column chromatography (PE/EtOAc 2:1). Colourless wax, $34.1 \mathrm{mg}, 95 \%$ yield. ${ }^{1} \mathbf{H}$ NMR (500 $\left.\mathrm{MHz}, \mathrm{CDCl}_{3}\right): \delta 7.65(\mathrm{~s}, 1 \mathrm{H}), 7.49(\mathrm{~d}, J=7.4 \mathrm{~Hz}, 1 \mathrm{H}), 7.46-7.35(\mathrm{~m}, 2 \mathrm{H}), 6.62(\mathrm{~s}$, 1H), 4.33-4.21 (m, 2H), $2.28(\mathrm{~s}, 3 \mathrm{H}), 2.04(\mathrm{~s}, 3 \mathrm{H}), 1.96(\mathrm{~s}, 3 \mathrm{H}), 1.26(\mathrm{t}, J=7.1 \mathrm{~Hz}$, $3 \mathrm{H}) ;{ }^{13} \mathrm{C}$ NMR $\left(126 \mathrm{MHz}, \mathrm{CDCl}_{3}\right): \delta 162.0,155.9,150.9,150.0,139.2,137.1,135.6$, $135.0,133.0,129.4,128.5,128.5,126.5,124.0,116.1,61.6,21.0,19.8,18.0,14.1$; HRMS (ESI): m/z calcd. for $\left[\mathrm{C}_{21} \mathrm{H}_{20} \mathrm{ClNNaO}_{4}, \mathrm{M}+\mathrm{Na}\right]^{+}: 408.0973$; found: 408.0975 .

Optical Rotation: $[\alpha]_{D}^{25}=+8.7(\mathrm{c}=0.3$, THF). The absolute configuration of $\mathbf{3 q}$ was assigned by analogy to 3a. $83 \%$ ee (HPLC condition: Chiralpak IB N-5 column, $n$-hexane $/ i-\operatorname{PrOH}=90: 10$, flow rate $=1 \mathrm{ml} / \mathrm{min}$, wavelength $=254 \mathrm{~nm}, \mathrm{t}_{\mathrm{R}}=8.0 \mathrm{~min}$ for minor isomer, $t_{R}=16.0 \mathrm{~min}$ for major isomer).

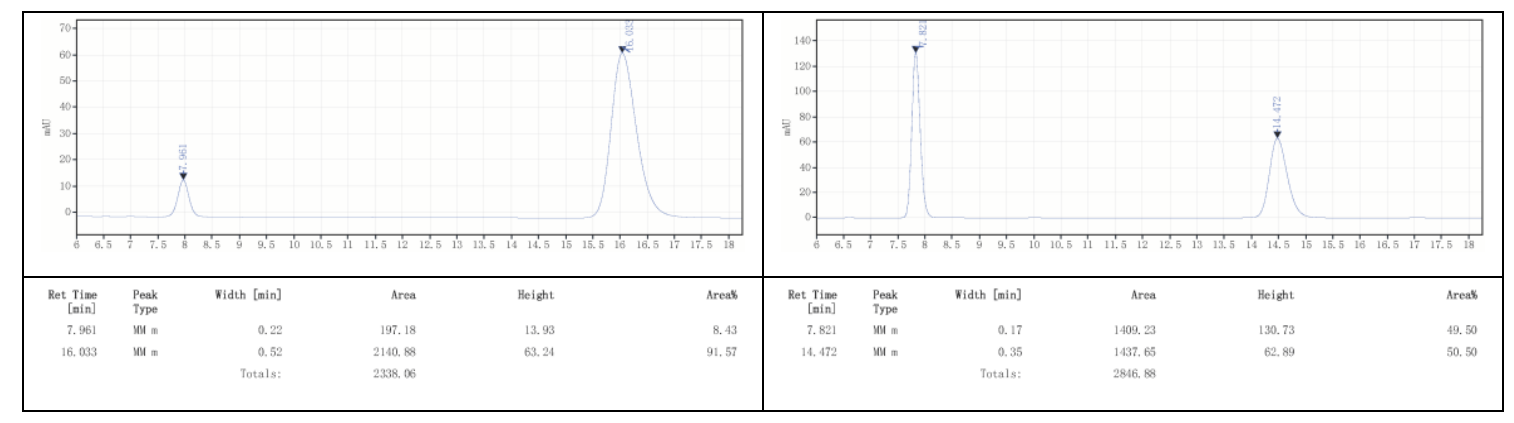

\section{Ethyl}

(R)-5-(2'-hydroxy-4',6'-dimethoxy-6-methyl-[1,1'-biphenyl]-2-yl)oxazole-4-carbo xylate (3r) 


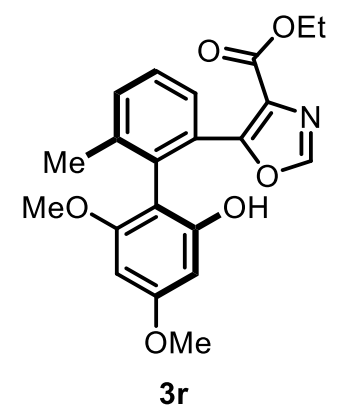

The general procedure outlined above was followed $(0.12 \mathrm{mmol}$ of $\mathbf{2 a}, 1.0 \mathrm{~mL}$ of $\mathrm{THF}$ at $-20{ }^{\circ} \mathrm{C}$ for $48 \mathrm{~h}$ ). The crude reaction mixture was purified by flash column chromatography (PE/EtOAc 2:1). Yellow wax, $29.9 \mathrm{mg}, 78 \%$ yield. ${ }^{1} \mathbf{H}$ NMR (500 $\mathrm{MHz}, \mathrm{CDCl}_{3}$ ): $\delta 7.66(\mathrm{~s}, 1 \mathrm{H}), 7.47$ (dd, $\left.J=7.4,0.8 \mathrm{~Hz}, 1 \mathrm{H}\right), 7.44-7.34$ (m, 2H), 6.06 (d, $J=2.3 \mathrm{~Hz}, 1 \mathrm{H}), 6.00(\mathrm{~d}, J=2.3 \mathrm{~Hz}, 1 \mathrm{H}), 5.21$ (br s, 1H), 4.33-4.22 (m, 2H), 3.74 (s, 3H), 3.59 (s, 3H), $2.13(\mathrm{~s}, 3 \mathrm{H}), 1.28(\mathrm{t}, J=7.1 \mathrm{~Hz}, 3 \mathrm{H}) ;{ }^{13} \mathrm{C}$ NMR $(126 \mathrm{MHz}$, $\left.\mathrm{CDCl}_{3}\right): \delta 162.1,161.2,158.1,156.6,154.4,149.8,140.3,132.9,132.7,129.6,128.8$, 128.2, 107.1, 93.5, 91.5, 61.4, 55.7, 55.3, 19.9, 14.2; HRMS (ESI): m/z calcd. for $\left[\mathrm{C}_{21} \mathrm{H}_{21} \mathrm{NNaO}_{6}, \mathrm{M}+\mathrm{Na}\right]^{+}$: 406.1261 ; found: 406.1263 .

Optical Rotation: $[\alpha]^{25}=-11.3(\mathrm{c}=0.3$, THF). The absolute configuration of $\mathbf{3 r}$ was assigned by analogy to 3a. 85\% ee (HPLC condition: Chiralpak IB N-5 column, $n$-hexane $/ i$-PrOH $=90: 10$, flow rate $=1 \mathrm{ml} / \mathrm{min}$, wavelength $=254 \mathrm{~nm}, \mathrm{t}_{\mathrm{R}}=11.1 \mathrm{~min}$ for minor isomer, $t_{R}=14.5 \mathrm{~min}$ for major isomer).

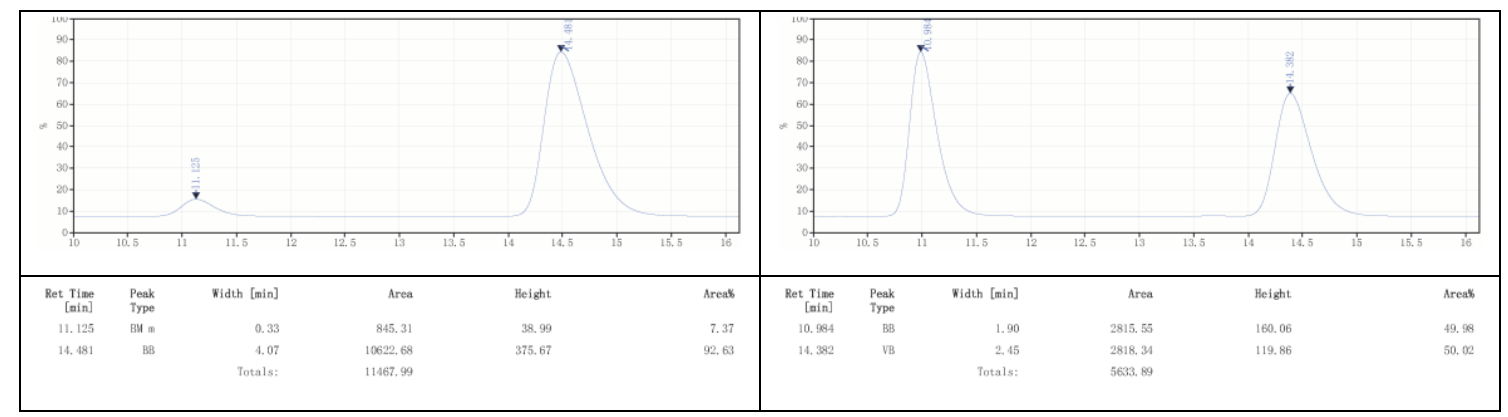

\section{Ethyl}

(R)-5-(2'-hydroxy-4',6'-dimethoxy-4,6-dimethyl-[1,1'-biphenyl]-2-yl)oxazole-4-ca rboxylate (3s) 


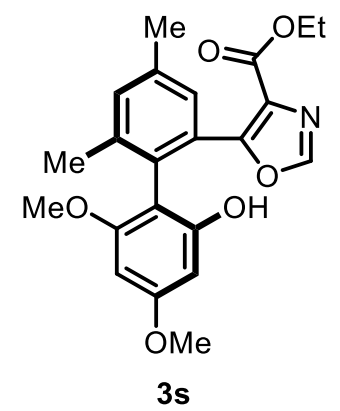

The general procedure outlined above was followed $(0.12 \mathrm{mmol}$ of $\mathbf{2 a}, 1.0 \mathrm{~mL}$ of $\mathrm{THF}$ at $-20{ }^{\circ} \mathrm{C}$ for $48 \mathrm{~h}$ ). The crude reaction mixture was purified by flash column chromatography (PE/EtOAc 2:1). White solid, $23.8 \mathrm{mg}, 60 \%$ yield. MP: $141-142{ }^{\circ} \mathrm{C}$; ${ }^{1} \mathbf{H}$ NMR $\left(400 \mathrm{MHz}, \mathrm{CDCl}_{3}\right): \delta 7.66(\mathrm{~s}, 1 \mathrm{H}), 7.29(\mathrm{~s}, 1 \mathrm{H}), 7.18(\mathrm{~s}, 1 \mathrm{H}), 6.05$ (d, $J=$ $1.9 \mathrm{~Hz}, 1 \mathrm{H}), 5.99$ (d, $J=1.9 \mathrm{~Hz}, 1 \mathrm{H}), 4.34-4.20(\mathrm{~m}, 2 \mathrm{H}), 3.73(\mathrm{~s}, 3 \mathrm{H}), 3.58(\mathrm{~s}, 3 \mathrm{H})$, $2.40(\mathrm{~s}, 3 \mathrm{H}), 2.09(\mathrm{~s}, 3 \mathrm{H}), 1.28(\mathrm{t}, J=7.1 \mathrm{~Hz}, 3 \mathrm{H}) ;{ }^{13} \mathrm{C}$ NMR $\left(101 \mathrm{MHz}, \mathrm{CDCl}_{3}\right): \delta$ $162.1,161.1,158.2$, 156.8, 154.6, 149.6, 140.0, 138.0, 133.7, 129.7, 129.4, 129.4, 128.0, 107.0, 93.4, 91.5, 61.4, 55.7, 55.3, 21.2, 19.8, 14.2; HRMS (ESI): m/z calcd. for $\left[\mathrm{C}_{22} \mathrm{H}_{23} \mathrm{NNaO}_{6}, \mathrm{M}+\mathrm{Na}\right]^{+}:$420.1418; found: 420.1418 .

Optical Rotation: $[\alpha]^{25}=+9.9(\mathrm{c}=0.3$, THF). The absolute configuration of $3 \mathrm{~s}$ was assigned by analogy to 3a. 91\% ee (HPLC condition: Chiralpak IB N-5 column, $n$-hexane $/ i$-PrOH $=90: 10$, flow rate $=1 \mathrm{ml} / \mathrm{min}$, wavelength $=254 \mathrm{~nm}, \mathrm{t}_{\mathrm{R}}=10.3 \mathrm{~min}$ for minor isomer, $t_{R}=12.4 \mathrm{~min}$ for major isomer).

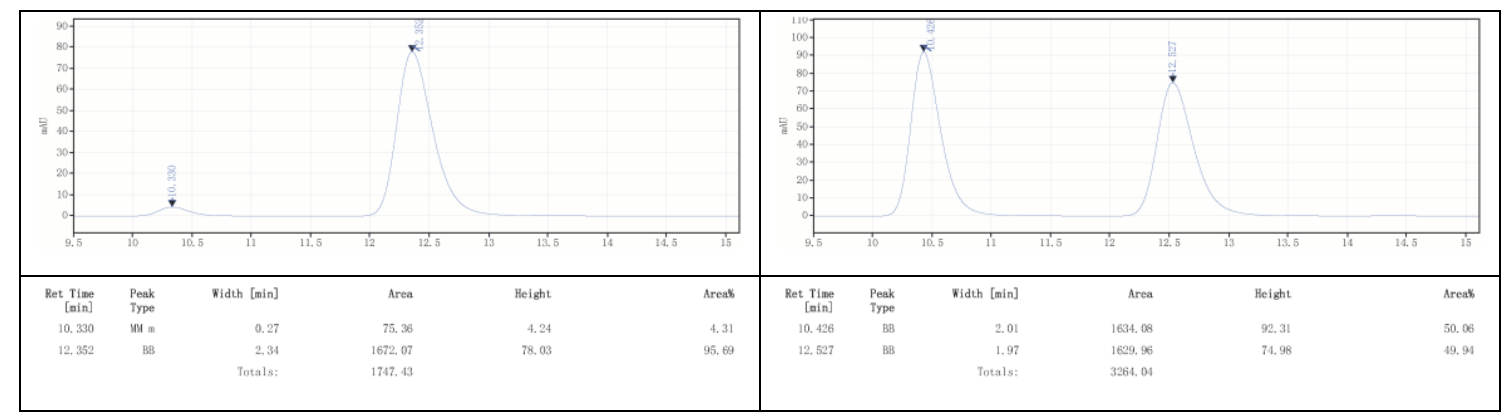

\section{Ethyl}

(R)-5-(6'-hydroxy-2',3',4'-trimethoxy-6-methyl-[1,1'-biphenyl]-2-yl)oxazole-4-car boxylate (3t) 


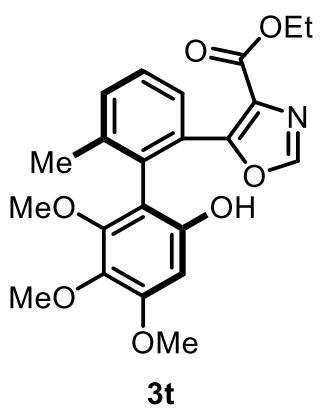

The general procedure outlined above was followed $(0.12 \mathrm{mmol}$ of $\mathbf{2 a}, 1.0 \mathrm{~mL}$ of $\mathrm{THF}$ at $-20{ }^{\circ} \mathrm{C}$ for $48 \mathrm{~h}$ ). The crude reaction mixture was purified by flash column chromatography (PE/EtOAc 2:1). Yellow wax, $24.0 \mathrm{mg}, 58 \%$ yield. ${ }^{1} \mathbf{H}$ NMR (500 $\left.\mathrm{MHz}, \mathrm{CDCl}_{3}\right): \delta 7.69(\mathrm{~s}, 1 \mathrm{H}), 7.48(\mathrm{~d}, J=7.2 \mathrm{~Hz}, 1 \mathrm{H}), 7.42(\mathrm{t}, J=7.6 \mathrm{~Hz}, 1 \mathrm{H})$, 7.39-7.34 (m, 1H), $6.21(\mathrm{~s}, 1 \mathrm{H}), 4.35-4.15(\mathrm{~m}, 2 \mathrm{H}), 3.79(\mathrm{~s}, 3 \mathrm{H}), 3.71(\mathrm{~s}, 3 \mathrm{H}), 3.61(\mathrm{~s}$, 3H), $2.17(\mathrm{~s}, 3 \mathrm{H}), 1.28(\mathrm{t}, J=7.1 \mathrm{~Hz}, 3 \mathrm{H}) ;{ }^{13} \mathbf{C}$ NMR $\left(126 \mathrm{MHz}, \mathrm{CDCl}_{3}\right): \delta 162.2$, $156.5,154.0,151.1,149.8,149.2,140.0,135.8,132.9$, 132.7, 129.3, 128.8, 128.4, 128.3, 111.7, 96.1, 61.5, 61.1, 60.6, 55.9, 20.1, 14.2; HRMS (ESI): m/z calcd. for $\left[\mathrm{C}_{22} \mathrm{H}_{23} \mathrm{NNaO}_{7}, \mathrm{M}+\mathrm{Na}\right]^{+}$: 436.1367; found: 436.1369 .

Optical Rotation: $[\alpha]^{25}=-13.8(\mathrm{c}=0.3$, THF $)$. The absolute configuration of $\mathbf{3 t}$ was assigned by analogy to 3a. 83\% ee (HPLC condition: Chiralpak IB N-5 column, $n$-hexane $/ i-\operatorname{PrOH}=90: 10$, flow rate $=1 \mathrm{ml} / \mathrm{min}$, wavelength $=254 \mathrm{~nm}, \mathrm{t}_{\mathrm{R}}=11.2 \mathrm{~min}$ for minor isomer, $t_{R}=16.1 \mathrm{~min}$ for major isomer).

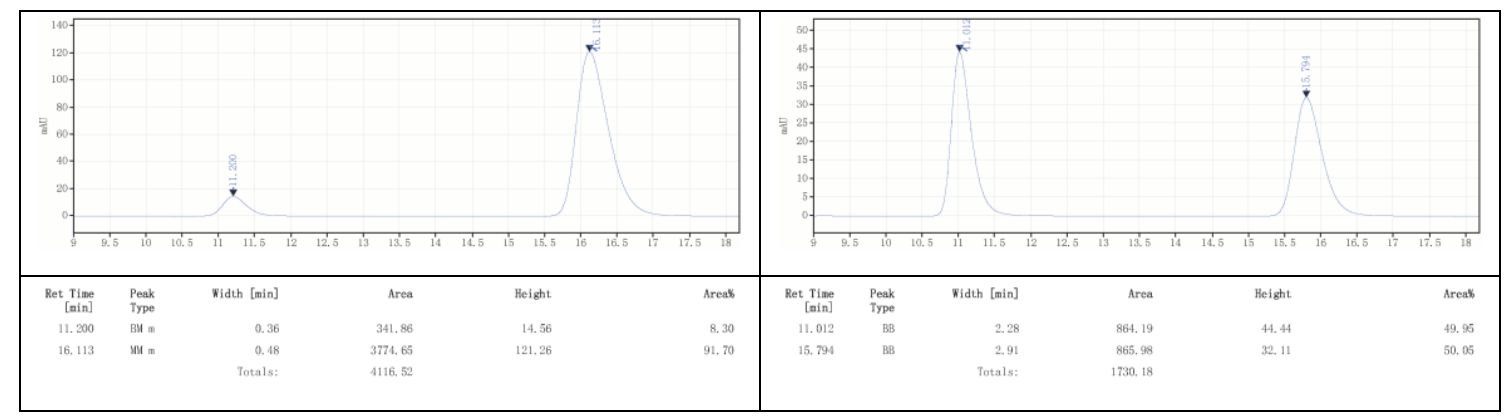

\section{Ethyl}

(R)-5-(2'-hydroxy-6'-methoxy-6-methyl-[1,1'-biphenyl]-2-yl)oxazole-4-carboxylat e $(3 \mathbf{u})$ 


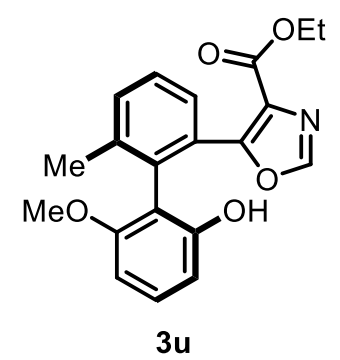

The general procedure outlined above was followed $(0.12 \mathrm{mmol}$ of $\mathbf{2 a}, 1.0 \mathrm{~mL}$ of THF at $-20{ }^{\circ} \mathrm{C}$ for $48 \mathrm{~h}$ ). The crude reaction mixture was purified by flash column chromatography (PE/EtOAc 2:1). White solid, $29.7 \mathrm{mg}, 84 \%$ yield. MP: $155-157{ }^{\circ} \mathrm{C}$; ${ }^{1} \mathbf{H}$ NMR $\left(500 \mathrm{MHz}, \mathrm{CDCl}_{3}\right): \delta 7.63(\mathrm{~s}, 1 \mathrm{H}), 7.51-7.47(\mathrm{~m}, 1 \mathrm{H}), 7.45-7.36(\mathrm{~m}, 2 \mathrm{H})$, $7.11(\mathrm{t}, J=8.3 \mathrm{~Hz}, 1 \mathrm{H}), 6.50(\mathrm{dd}, J=8.2,0.9 \mathrm{~Hz}, 1 \mathrm{H}), 6.40(\mathrm{dd}, J=8.3,0.7 \mathrm{~Hz}, 1 \mathrm{H})$, $4.27(\mathrm{qd}, J=7.1,0.5 \mathrm{~Hz}, 2 \mathrm{H}), 3.61(\mathrm{~s}, 3 \mathrm{H}), 2.13(\mathrm{~s}, 3 \mathrm{H}), 1.27(\mathrm{t}, J=7.1 \mathrm{~Hz}, 3 \mathrm{H}) ;{ }^{13} \mathrm{C}$ NMR (126 MHz, $\left.\mathrm{CDCl}_{3}\right): \delta 162.0,157.4,156.4,153.8,149.7,139.7,133.0,132.8$, 129.7, 129.1, 128.9, 128.3, 128.2, 114.4, 109.2, 102.9, 61.4, 55.7, 19.9, 14.2; HRMS (ESI): $\mathrm{m} / \mathrm{z}$ calcd. for $\left[\mathrm{C}_{20} \mathrm{H}_{19} \mathrm{NNaO}_{5}, \mathrm{M}+\mathrm{Na}\right]^{+}: 376.1155$; found: 376.1156 .

Optical Rotation: $[\alpha]^{25}=-13.6(\mathrm{c}=0.3$, THF). The absolute configuration of $\mathbf{3 u}$ was assigned by analogy to 3a. $89 \%$ ee (HPLC condition: Chiralpak IB N-5 column, $n$-hexane $/ i-\operatorname{PrOH}=90: 10$, flow rate $=1 \mathrm{ml} / \mathrm{min}$, wavelength $=254 \mathrm{~nm}, \mathrm{t}_{\mathrm{R}}=11.7 \mathrm{~min}$ for minor isomer, $t_{R}=13.7 \mathrm{~min}$ for major isomer).

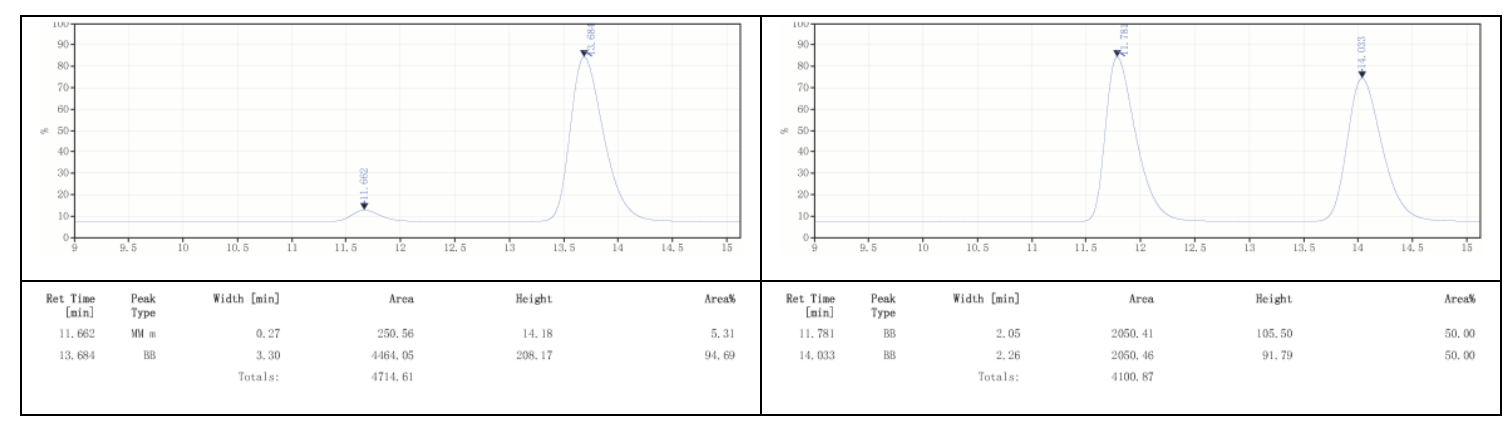

\section{Ethyl}

(R)-5-(6-ethyl-2'-hydroxy-6'-methoxy-[1,1'-biphenyl]-2-yl)oxazole-4-carboxylate $(3 \mathbf{v})$ 


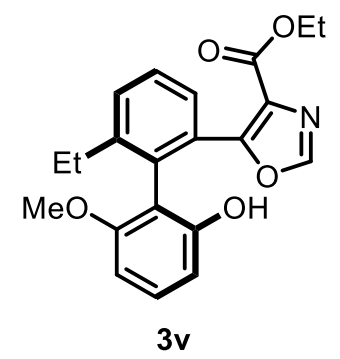

The general procedure outlined above was followed $(0.12 \mathrm{mmol}$ of $\mathbf{2 a}, 1.0 \mathrm{~mL}$ of $\mathrm{THF}$ at $-20{ }^{\circ} \mathrm{C}$ for $48 \mathrm{~h}$ ). The crude reaction mixture was purified by flash column chromatography (PE/EtOAc 2:1). Yellow wax, $32.0 \mathrm{mg}$, 87\% yield. ${ }^{1} \mathbf{H}$ NMR (500 $\left.\mathrm{MHz}, \mathrm{CDCl}_{3}\right): \delta 7.62(\mathrm{~s}, 1 \mathrm{H}), 7.53(\mathrm{dd}, J=7.7,1.3 \mathrm{~Hz}, 1 \mathrm{H}), 7.48(\mathrm{t}, J=7.7 \mathrm{~Hz}, 1 \mathrm{H})$, $7.40(\mathrm{dd}, J=7.6,1.4 \mathrm{~Hz}, 1 \mathrm{H}), 7.11(\mathrm{t}, J=8.3 \mathrm{~Hz}, 1 \mathrm{H}), 6.49(\mathrm{dd}, J=8.2,0.9 \mathrm{~Hz}, 1 \mathrm{H})$, $6.40(\mathrm{dd}, J=8.3,0.7 \mathrm{~Hz}, 1 \mathrm{H}), 4.33-4.19(\mathrm{~m}, 2 \mathrm{H}), 3.62$ (s, 3H), 2.43 (q, $J=7.6 \mathrm{~Hz}$, $2 \mathrm{H}), 1.27(\mathrm{t}, J=7.1 \mathrm{~Hz}, 3 \mathrm{H}), 1.06(\mathrm{t}, J=7.6 \mathrm{~Hz}, 3 \mathrm{H}) ;{ }^{13} \mathbf{C}$ NMR $\left(126 \mathrm{MHz}, \mathrm{CDCl}_{3}\right)$ : $\delta 162.0,157.6,156.4,154.0,149.7,145.5,132.3,131.2,129.7,129.1,129.0,128.5$, 128.3, 114.1, 109.1, 102.8, 61.4, 55.7, 26.4, 14.8, 14.2; HRMS (ESI): m/z calcd. for $\left[\mathrm{C}_{21} \mathrm{H}_{21} \mathrm{NNaO}_{5}, \mathrm{M}+\mathrm{Na}\right]^{+}: 390.1312$; found: 390.1313 .

Optical Rotation: $[\alpha]_{D}^{25}=-17.6(\mathrm{c}=0.3$, THF). The absolute configuration of $\mathbf{3 v}$ was assigned by analogy to 3a. 92\% ee (HPLC condition: Chiralpak IB N-5 column, $n$-hexane $/ i-\operatorname{PrOH}=90: 10$, flow rate $=1 \mathrm{ml} / \mathrm{min}$, wavelength $=254 \mathrm{~nm}, \mathrm{t}_{\mathrm{R}}=10.2 \mathrm{~min}$ for minor isomer, $t_{R}=11.9$ min for major isomer).

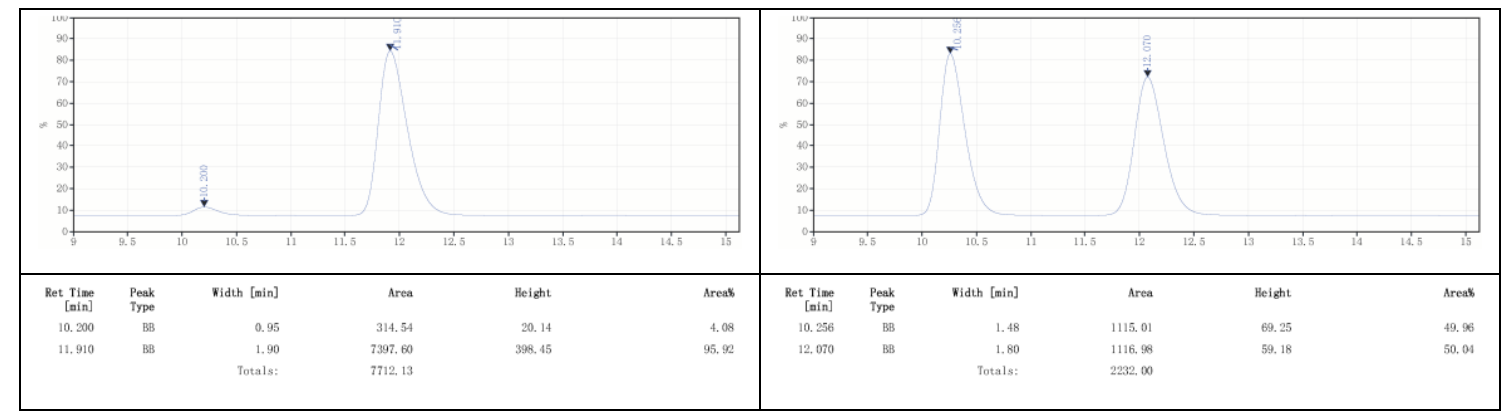

\section{Ethyl}

(R)-5-(2'-hydroxy-6'-methoxy-4,6-dimethyl-[1,1'-biphenyl]-2-yl)oxazole-4-carbox ylate (3w) 


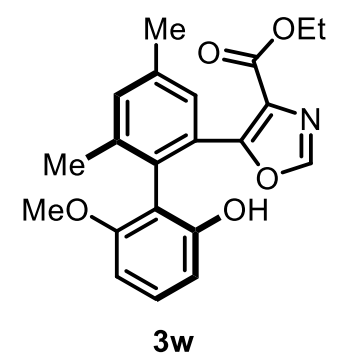

The general procedure outlined above was followed $(0.12 \mathrm{mmol}$ of $\mathbf{2 a}, 1.0 \mathrm{~mL}$ of THF at $-20{ }^{\circ} \mathrm{C}$ for $48 \mathrm{~h}$ ). The crude reaction mixture was purified by flash column chromatography (PE/EtOAc 2:1). White solid, $28.7 \mathrm{mg}$, 78\% yield. MP: 132-133 ${ }^{\circ} \mathrm{C}$; ${ }^{1} \mathbf{H}$ NMR (500 MHz, $\left.\mathrm{CDCl}_{3}\right): \delta 7.63(\mathrm{~s}, 1 \mathrm{H}), 7.31(\mathrm{~s}, 1 \mathrm{H}), 7.21(\mathrm{~s}, 1 \mathrm{H}), 7.09$ (t, $J=8.3$ $\mathrm{Hz}, 1 \mathrm{H}), 6.49$ (dd, $J=8.2,0.8 \mathrm{~Hz}, 1 \mathrm{H}), 6.39$ (dd, $J=8.3,0.5 \mathrm{~Hz}, 1 \mathrm{H}), 5.15$ (br s, $1 \mathrm{H}$ ), 4.33-4.19 (m, 2H), 3.60 (s, 3H), 2.41 (s, 3H), 2.09 (s, 3H), 1.28 (t, J = 7.1 Hz, 3H); ${ }^{13} \mathrm{C}$ NMR $\left(126 \mathrm{MHz}, \mathrm{CDCl}_{3}\right): \delta 162.0,157.4,156.6,154.0,149.6,139.4,138.1$, 133.7, 129.8, 129.6, 129.4, 128.9, 128.0, 114.3, 109.1, 102.9, 61.4, 55.7, 21.2, 19.8, 14.2; HRMS (ESI): $\mathrm{m} / \mathrm{z}$ calcd. for $\left[\mathrm{C}_{21} \mathrm{H}_{21} \mathrm{NNaO}_{5}, \mathrm{M}+\mathrm{Na}\right]^{+}$: 390.1312; found: 390.1312 .

Optical Rotation: $[\alpha]^{25}{ }_{\mathrm{D}}=-139.3(\mathrm{c}=0.3$, THF). The absolute configuration of $\mathbf{3 w}$ was assigned by analogy to 3a. 92\% ee (HPLC condition: Chiralpak IA column, $n$-hexane $/ i-\mathrm{PrOH}=90: 10$, flow rate $=1 \mathrm{ml} / \mathrm{min}$, wavelength $=254 \mathrm{~nm}, \mathrm{t}_{\mathrm{R}}=9.1 \mathrm{~min}$ for major isomer, $t_{R}=11.6 \mathrm{~min}$ for minor isomer).

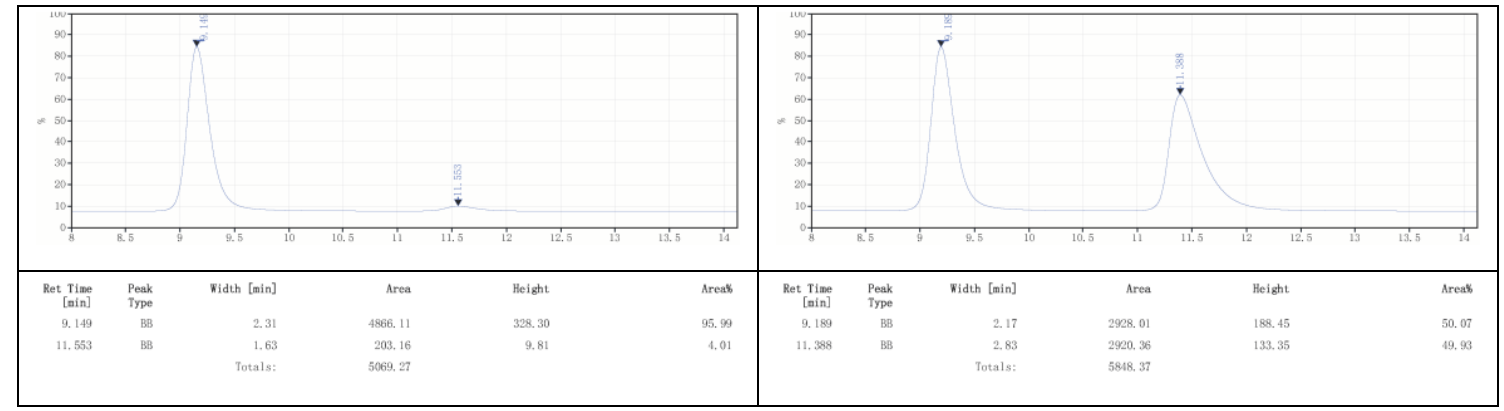

Ethyl (S)-5-(2'-hydroxy-[1,1'-binaphthalen]-2-yl)oxazole-4-carboxylate (3x)<smiles>CCOC(=O)c1ncoc1-c1ccc2ccccc2c1-c1c(O)ccc2ccccc12</smiles>

$3 \mathbf{x}$ 
The general procedure outlined above was followed ( $1 \mathrm{~mol} \% \mathrm{Ag}_{2} \mathrm{CO}_{3}, 2 \mathrm{~mol} \% \mathbf{L 5}$, $0.50 \mathrm{mmol}$ of $\mathbf{1 x}, 0.50 \mathrm{mmol}$ of $\mathbf{2 a}, 25.0 \mathrm{~mL}$ of THF at $40{ }^{\circ} \mathrm{C}$ for $48 \mathrm{~h}$ ). The crude reaction mixture was purified by flash column chromatography (PE/EtOAc 2:1). White solid, $165.6 \mathrm{mg}, 81 \%$ yield. MP: $210-211{ }^{\circ} \mathrm{C} ;{ }^{1} \mathbf{H}$ NMR $\left(500 \mathrm{MHz}, \mathrm{CDCl}_{3}\right): \delta$ $8.10(\mathrm{~d}, J=8.6 \mathrm{~Hz}, 1 \mathrm{H}), 8.01(\mathrm{~d}, J=8.2 \mathrm{~Hz}, 1 \mathrm{H}), 7.78(\mathrm{t}, J=9.0 \mathrm{~Hz}, 2 \mathrm{H}), 7.69(\mathrm{~d}, J=$ 8.5 Hz, 1H), 7.62-7.56 (m, 1H), 7.43 (s, 1H), 7.39-7.30 (m, 2H), 7.29-7.26 (m, 1H), 7.22-7.16 (m, 2H), 7.08-7.02 (m, 1H), $5.46(\mathrm{br} \mathrm{s}, 1 \mathrm{H}), 4.34-4.22(\mathrm{~m}, 2 \mathrm{H}), 1.28(\mathrm{t}, J=$ $7.1 \mathrm{~Hz}, 3 \mathrm{H}) ;{ }^{13} \mathbf{C} \mathbf{N M R}\left(126 \mathrm{MHz}, \mathrm{CDCl}_{3}\right): \delta 161.9,155.9,151.6,150.0,134.7,133.8$, $133.6,132.8,130.4$, 129.1, 128.7, 128.7, 128.5, 128.0, 127.8, 127.6, 127.3, 126.7, 126.6, 124.9, 123.5, 118.0, 116.4, 61.6, 14.2; HRMS (ESI): $\mathrm{m} / \mathrm{z}$ calcd. for $\left[\mathrm{C}_{26} \mathrm{H}_{19} \mathrm{NNaO}_{4}, \mathrm{M}+\mathrm{Na}\right]^{+}$: 432.1206; found: 432.1208 .

Optical Rotation: $[\alpha]^{25}=-28.7(\mathrm{c}=0.3$, THF). The absolute configuration of $\mathbf{3 x}$ was assigned by analogy to 3a. 84\% ee (HPLC condition: Chiralpak IB N-5 column, $n$-hexane $/ i$-PrOH $=90: 10$, flow rate $=1 \mathrm{ml} / \mathrm{min}$, wavelength $=254 \mathrm{~nm}, \mathrm{t}_{\mathrm{R}}=15.1 \mathrm{~min}$ for minor isomer, $t_{R}=19.3 \mathrm{~min}$ for major isomer).

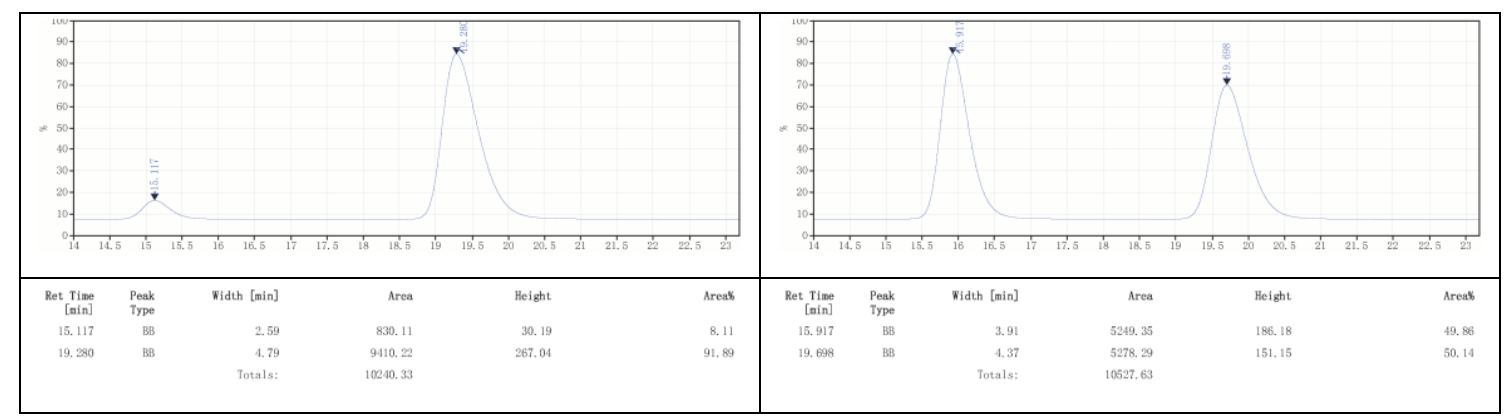

\section{Methyl}

(S)-5-(1-(2-hydroxy-4,6-dimethylphenyl)naphthalen-2-yl)oxazole-4-carboxylate (4a)<smiles>COC(=O)c1ncoc1-c1ccc2ccccc2c1-c1c(C)cc(C)cc1O</smiles>

The general procedure outlined above was followed $(0.10 \mathrm{mmol}$ of $\mathbf{2 b}, 1.0 \mathrm{~mL}$ of THF at $-20{ }^{\circ} \mathrm{C}$ for $48 \mathrm{~h}$ ). The crude reaction mixture was purified by flash column 
chromatography (PE/EtOAc 2:1). White solid, $36.6 \mathrm{mg}$, 98\% yield. MP: $123-125{ }^{\circ} \mathrm{C}$; ${ }^{1} \mathbf{H}$ NMR $\left(500 \mathrm{MHz}, \mathrm{CDCl}_{3}\right): \delta 8.01(\mathrm{~d}, J=8.5 \mathrm{~Hz}, 1 \mathrm{H}), 7.96(\mathrm{~d}, J=8.2 \mathrm{~Hz}, 1 \mathrm{H})$, $7.69(\mathrm{~s}, 1 \mathrm{H}), 7.62-7.55(\mathrm{~m}, 2 \mathrm{H}), 7.51-7.43(\mathrm{~m}, 2 \mathrm{H}), 6.62(\mathrm{~s}, 1 \mathrm{H}), 6.58(\mathrm{~s}, 1 \mathrm{H}), 4.89$ (br s, 1H), 3.82 (s, 3H), 2.28 (s, 3H), 1.83 (s, 3H); $\left.{ }^{13} \mathbf{C ~ N M R ~ ( 1 2 6 ~ M H z , ~} \mathrm{CDCl}_{3}\right): \delta 162.4$, $156.4,153.5,150.1,139.4,138.1,135.1,134.7,132.5,128.7,128.4,128.4,127.9$, 127.6, 127.6, 126.3, 126.1, 123.0, 120.5, 114.1, 52.4, 21.4, 19.9; HRMS (ESI): m/z calcd. for $\left[\mathrm{C}_{23} \mathrm{H}_{19} \mathrm{NNaO}_{4}, \mathrm{M}+\mathrm{Na}\right]^{+}:$396.1206; found: 396.1206.

Optical Rotation: $[\alpha]^{25}{ }_{\mathrm{D}}=+60.8(\mathrm{c}=0.3$, THF). The absolute configuration of $4 \mathbf{a}$ was assigned by analogy to 3a. 92\% ee (HPLC condition: Chiralpak IA column, $n$-hexane $/ i-\mathrm{PrOH}=90: 10$, flow rate $=1 \mathrm{ml} / \mathrm{min}$, wavelength $=254 \mathrm{~nm}, \mathrm{t}_{\mathrm{R}}=10.2 \mathrm{~min}$ for major isomer, $\mathrm{t}_{\mathrm{R}}=11.9 \mathrm{~min}$ for minor isomer).

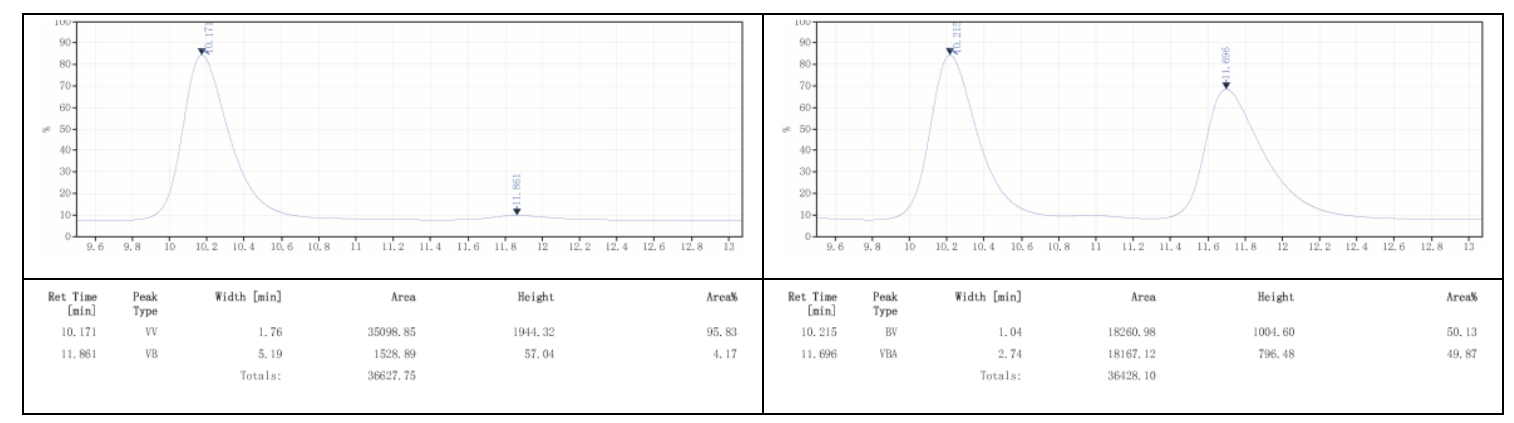

\section{tert-Butyl}

(S)-5-(1-(2-hydroxy-4,6-dimethylphenyl)naphthalen-2-yl)oxazole-4-carboxylate (4b)<smiles>CCCCOC(=O)c1ncoc1-c1ccc2ccccc2c1-c1c(C)cc([N+](=O)[O-])cc1O</smiles>

The general procedure outlined above was followed $(0.10 \mathrm{mmol}$ of $2 \mathrm{c}, 1.0 \mathrm{~mL}$ of THF at $-20{ }^{\circ} \mathrm{C}$ for $48 \mathrm{~h}$ ). The crude reaction mixture was purified by flash column chromatography (PE/EtOAc 2:1). White solid, $36.1 \mathrm{mg}, 87 \%$ yield. MP: $116-118{ }^{\circ} \mathrm{C}$; ${ }^{1} \mathbf{H}$ NMR (500 MHz, $\left.\mathrm{CDCl}_{3}\right): \delta 8.00(\mathrm{~d}, J=8.5 \mathrm{~Hz}, 1 \mathrm{H}), 7.95(\mathrm{~d}, J=8.2 \mathrm{~Hz}, 1 \mathrm{H})$, 7.67 (s, 1H), 7.63-7.54 (m, 2H), 7.52-7.40 (m, 2H), 6.61 (s, 1H), 6.59 (s, 1H), 4.84 (br s, 1H), $2.28(\mathrm{~s}, 3 \mathrm{H}), 1.83(\mathrm{~s}, 3 \mathrm{H}), 1.41(\mathrm{~s}, 9 \mathrm{H}) ;{ }^{13} \mathbf{C ~ N M R}\left(126 \mathrm{MHz}, \mathrm{CDCl}_{3}\right): \delta 160.9$, 
$155.4,153.5,150.1,139.4,138.0,135.0,134.5,132.5,129.8,128.5,128.4,128.1$, 127.8, 127.5, 126.7, 126.2, 123.0, 120.7, 114.1, 82.8, 28.1, 21.4, 19.9; HRMS (ESI): $\mathrm{m} / \mathrm{z}$ calcd. for $\left[\mathrm{C}_{26} \mathrm{H}_{25} \mathrm{NNaO}_{4}, \mathrm{M}+\mathrm{Na}\right]^{+}:$438.1676; found: 438.1677 .

Optical Rotation: $[\alpha]^{25} \mathrm{D}=+49.9(\mathrm{c}=0.4$, THF). The absolute configuration of $\mathbf{4 b}$ was assigned by analogy to 3a. 90\% ee (HPLC condition: Chiralpak IC column, $n$-hexane $/ i-\mathrm{PrOH}=90: 10$, flow rate $=1 \mathrm{ml} / \mathrm{min}$, wavelength $=254 \mathrm{~nm}, \mathrm{t}_{\mathrm{R}}=12.0 \mathrm{~min}$ for major isomer, $t_{R}=16.1 \mathrm{~min}$ for minor isomer).

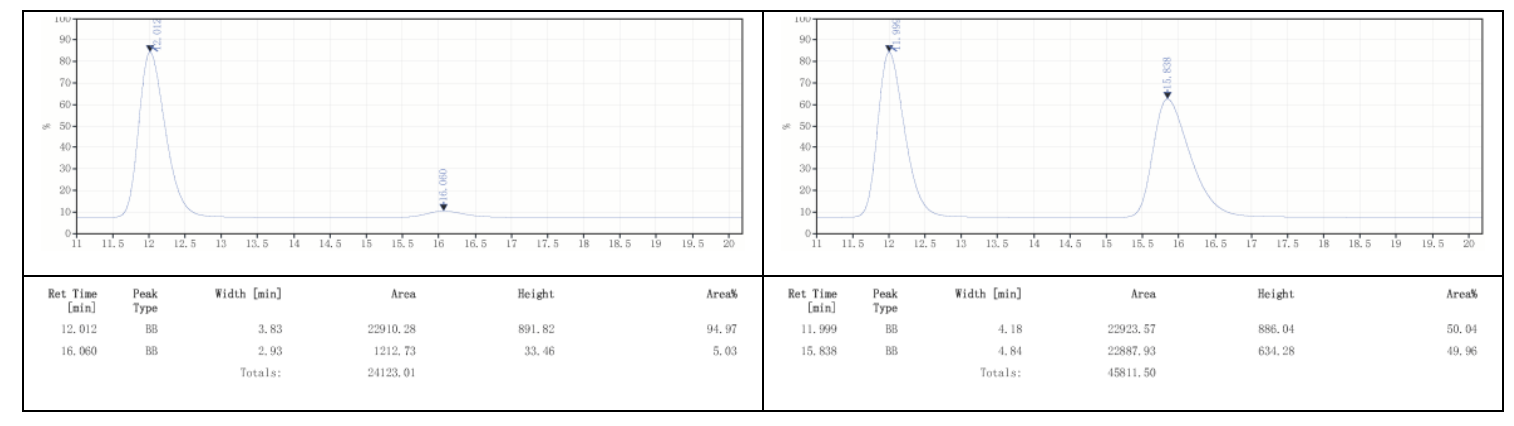

(S)-4-Chloro-3,5-dimethyl-2-(2-(4-tosyloxazol-5-yl)naphthalen-1-yl)phenol (4c)<smiles>Cc1ccc(S(=O)(=O)c2ncoc2-c2ccc3ccccc3c2-c2c(O)cc(C)c(Cl)c2C)cc1</smiles>

The general procedure outlined above was followed $(0.15 \mathrm{mmol}$ of $\mathbf{2 d}, 5.0 \mathrm{~mL}$ of THF at $24{ }^{\circ} \mathrm{C}$ for $48 \mathrm{~h}$ ). The crude reaction mixture was purified by flash column chromatography (PE/EtOAc 2:1). White solid, $31.2 \mathrm{mg}$, 62\% yield. MP: 232-234 ${ }^{\circ} \mathrm{C}$; ${ }^{1} \mathbf{H}$ NMR $\left(500 \mathrm{MHz}, \mathrm{CDCl}_{3}\right): \delta 8.08(\mathrm{~d}, J=8.5 \mathrm{~Hz}, 1 \mathrm{H}), 8.00(\mathrm{~d}, J=8.2 \mathrm{~Hz}, 1 \mathrm{H})$, $7.94(\mathrm{~d}, J=8.3 \mathrm{~Hz}, 2 \mathrm{H}), 7.69(\mathrm{~d}, J=8.5 \mathrm{~Hz}, 1 \mathrm{H}), 7.66(\mathrm{~s}, 1 \mathrm{H}), 7.65-7.61(\mathrm{~m}, 1 \mathrm{H})$, 7.51-7.47 (m, 1H), $7.44(\mathrm{~d}, J=8.5 \mathrm{~Hz}, 1 \mathrm{H}), 7.37(\mathrm{~d}, J=8.0 \mathrm{~Hz}, 2 \mathrm{H}), 6.72(\mathrm{~s}, 1 \mathrm{H})$, 5.09 (br s, 1H), 2.45 (s, 3H), 2.36 (s, 3H), 1.98 (s, 3H); $\left.{ }^{13} \mathbf{C ~ N M R ~ ( 1 2 6 ~ M H z , ~} \mathrm{CDCl}_{3}\right)$ : $\delta 152.8,151.5,150.6,145.5,137.9,137.6,136.4,136.3,134.9,134.8,132.2,130.0$, 129.2, 128.8, 128.6, 128.4, 128.2, 128.0, 126.5, 126.2, 124.8, 122.4, 116.2, 21.9, 21.1, 
18.6; HRMS (ESI): $\mathrm{m} / \mathrm{z}$ calcd. for $\left[\mathrm{C}_{28} \mathrm{H}_{22} \mathrm{ClNNaO}_{4} \mathrm{~S}, \mathrm{M}+\mathrm{Na}\right]^{+}:$526.0850; found: 526.0850 .

Optical Rotation: $[\alpha]^{25}=+23.1(\mathrm{c}=0.4$, THF). The absolute configuration of $4 \mathbf{c}$ was assigned by analogy to 3a. $84 \%$ ee (HPLC condition: Chiralpak IC column, $n$-hexane $/ i-\operatorname{PrOH}=80: 20$, flow rate $=1 \mathrm{ml} / \mathrm{min}$, wavelength $=254 \mathrm{~nm}, \mathrm{t}_{\mathrm{R}}=15.8 \mathrm{~min}$ for major isomer, $t_{R}=18.5 \mathrm{~min}$ for minor isomer).

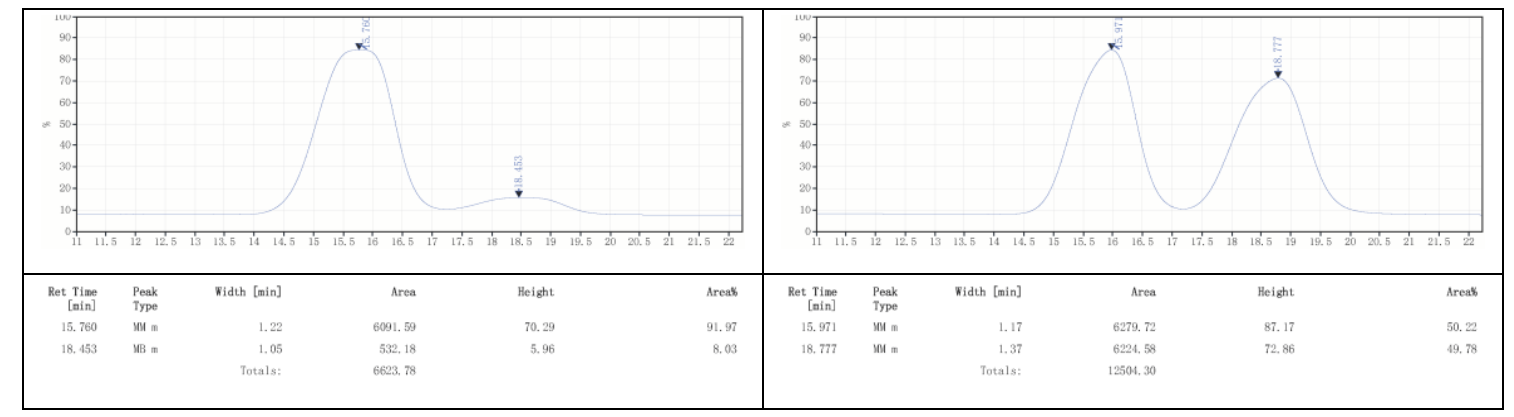

(S)-(5-(3'-Chloro-6'-hydroxy-2',4',6-trimethyl-[1,1'-biphenyl]-2-yl)oxazol-4-yl)(m orpholino)methanone (4d)<smiles>Cc1cc(O)c(-c2c(C)cccc2-c2ocnc2C(=O)N2CCOCC2)c(C)c1Cl</smiles>

The general procedure outlined above was followed $(0.10 \mathrm{mmol}$ of $\mathbf{2 e}, 5.0 \mathrm{~mL}$ of THF at $24{ }^{\circ} \mathrm{C}$ for $24 \mathrm{~h}$ ). The crude reaction mixture was purified by flash column chromatography (PE/EtOAc 2:1). Pale yellow wax, $33.3 \mathrm{mg}, 78 \%$ yield. ${ }^{1} \mathbf{H}$ NMR $\left(500 \mathrm{MHz}, \mathrm{CDCl}_{3}\right): \delta 7.66(\mathrm{~s}, 1 \mathrm{H}), 7.45(\mathrm{~d}, J=7.6 \mathrm{~Hz}, 1 \mathrm{H}), 7.40(\mathrm{t}, J=7.6 \mathrm{~Hz}, 1 \mathrm{H})$, $7.29(\mathrm{~d}, J=7.5 \mathrm{~Hz}, 1 \mathrm{H}), 6.68(\mathrm{~s}, 1 \mathrm{H}), 3.84-3.78(\mathrm{~m}, 1 \mathrm{H}), 3.76-3.59(\mathrm{~m}, 7 \mathrm{H}), 2.31(\mathrm{~s}$, 3H), 2.01 (s, 3H), 1.97 (s, 3H); $\left.{ }^{13} \mathbf{C ~ N M R ~ ( 1 2 6 ~ M H z , ~} \mathrm{CDCl}_{3}\right): \delta 161.9,155.0,151.5$, $148.8,139.0,136.8,136.6,135.1,132.7,131.1,128.7$, 128.5, 128.0, 126.7, 126.0, 118.2, 67.0, 66.8, 47.4, 43.0, 21.0, 19.9, 18.2; HRMS (ESI): $\mathrm{m} / \mathrm{z}$ calcd. for $\left[\mathrm{C}_{23} \mathrm{H}_{23} \mathrm{ClN}_{2} \mathrm{NaO}_{4}, \mathrm{M}+\mathrm{Na}\right]^{+}$: 449.1239; found: 449.1239 .

Optical Rotation: $[\alpha]^{25}=-75.1(\mathrm{c}=0.1$, THF $)$. The absolute configuration of $4 \mathbf{d}$ 
was assigned by analogy to 3a. 70\% ee (HPLC condition: Chiralpak IC column, $n$-hexane $/ i-\operatorname{PrOH}=85: 15$, flow rate $=1 \mathrm{ml} / \mathrm{min}$, wavelength $=254 \mathrm{~nm}, \mathrm{t}_{\mathrm{R}}=16.8 \mathrm{~min}$ for minor isomer, $t_{R}=18.8 \mathrm{~min}$ for major isomer).

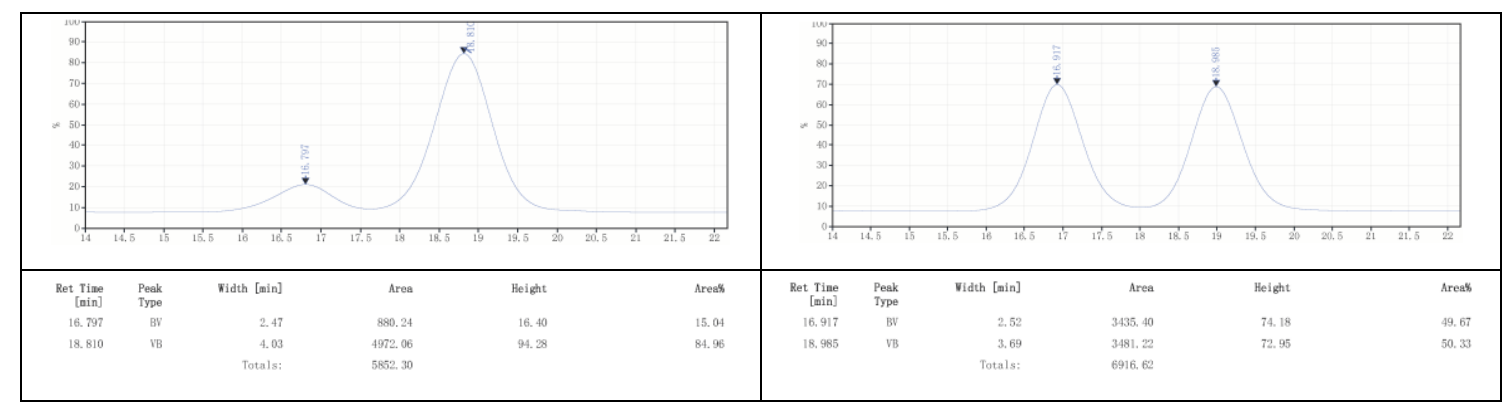

\section{DFT calculations}

\section{Complete reference for Gaussian 09}

Frisch, M. J.; Trucks, G. W.; Schlegel, H. B.; Scuseria, G. E.; Robb, M. A.; Cheeseman, J. R.; Scalmani, G.; Barone, V.; Mennucci, B.; Petersson, G. A.; Nakatsuji, H.; Caricato, M.; Li, X.; Hratchian, H. P.; Izmaylov, A. F.; Bloino, J.; Zheng, G.; Sonnenberg, J. L.; Hada, M.; Ehara, M.; Toyota, K.; Fukuda, R.; Hasegawa, J.; Ishida, M.; Nakajima, T.; Honda, Y.; Kitao, O.; Nakai, H.; Vreven, T.; Montgomery, Jr., J. A.; Peralta, J. E.; Ogliaro, F.; Bearpark, M.; Heyd, J. J.; Brothers, E.; Kudin, K. N.; Staroverov, V. N.; Keith, T.; Kobayashi, R.; Normand, J.; Raghavachari, K.; Rendell, A.; Burant, J. C.; Iyengar, S. S.; Tomasi, J.; Cossi, M.; Rega, N.; Millam, J. M.; Klene, M.; Knox, J. E.; Cross, J. B.; Bakken, V.; Adamo, C.; Jaramillo, J.; Gomperts, R.; Stratmann, R. E.; Yazyev, O.; Austin, A. J.; Cammi, R.; Pomelli, C.; Ochterski, J. W.; Martin, R. L.; Morokuma, K.; Zakrzewski, V. G.; Voth, G. A.; Salvador, P.; Dannenberg, J. J.; Dapprich, S.; Daniels, A. D.; Farkas, O.; Foresman, J. B.; Ortiz, J. V.; Cioslowski, J.; and Fox, D. J. Gaussian 09, revision D.01; Gaussian, Inc.: Wallingford, CT, 2013.

\section{Computational methods}

All the DFT calculations were carried out with the Gaussian 09 series of programs. The B3-LYP ${ }^{4}$ functional with the standard 6-31G(d) basis set was used for geometry optimizations. Harmonic vibrational frequency calculations were performed for all stationary points to determine whether they are local minima or transition structure and to derive thermochemical corrections for the enthalpies and free energies. The M06 functional ${ }^{5}$ proposed by Truhlar et al. with the $6-311+\mathrm{g}(\mathrm{d})$ basis set was used to 
calculate the single point energies, because it is expected that this strategy will provide greater accuracy with regard to the energetic information. ${ }^{6}$ The solvent effects were considered by single-point calculations of the gas-phase stationary points with the SMD continuum model. ${ }^{7}$ The energies reported in this paper are the M06 calculated Gibbs free energies in chlorobenzene solvent based on B3LYP calculated geometries with thermodynamic corrections calculated at the same level.

\section{Enantiomeric conversion half-life calculation strategy}

The Eyring Equation relates the activation free energy and rate constant:

$$
k=\frac{\kappa k_{b} T}{h} e^{-\frac{\Delta G^{\ddagger}}{R T}}
$$

In this equation, $\Delta G^{\neq}$is the Gibbs energy of activation, $\kappa$ is the transmission coefficient, $k_{b}$ is Boltzmann's constant, and $h$ is Planck's constant. The transmission coefficient is often assumed to be equal to one as it reflects what fraction of the flux through the transition state proceeds to the product without recrossing the transition state.

The epimerization of atropisomers is a first order reaction, which makes the half-life only relates to the reaction rate constant:

$$
t_{1 / 2}=\ln 2 / 2 k
$$

\section{Model reaction for half-life calculation}




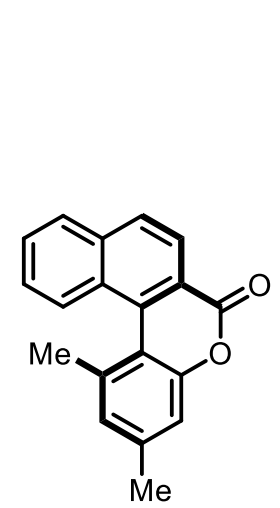

$(R)-1 \mathrm{a}$

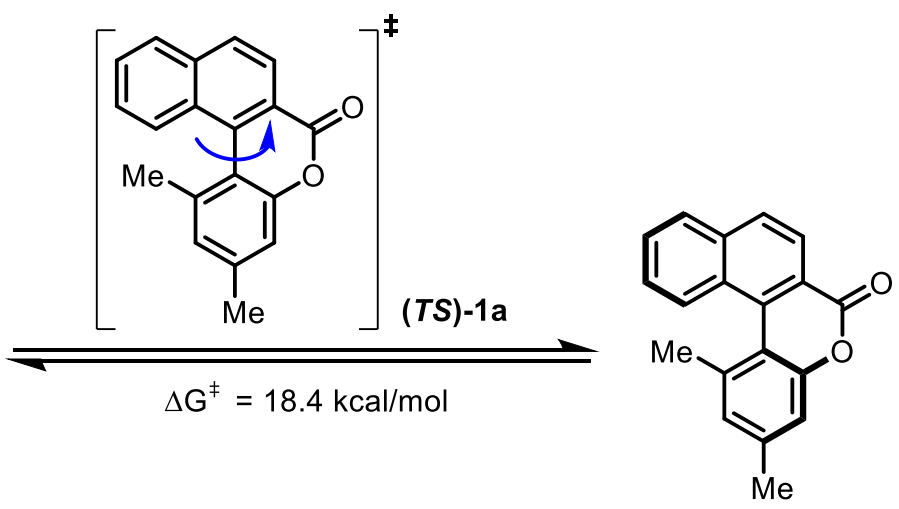

(S)-1a

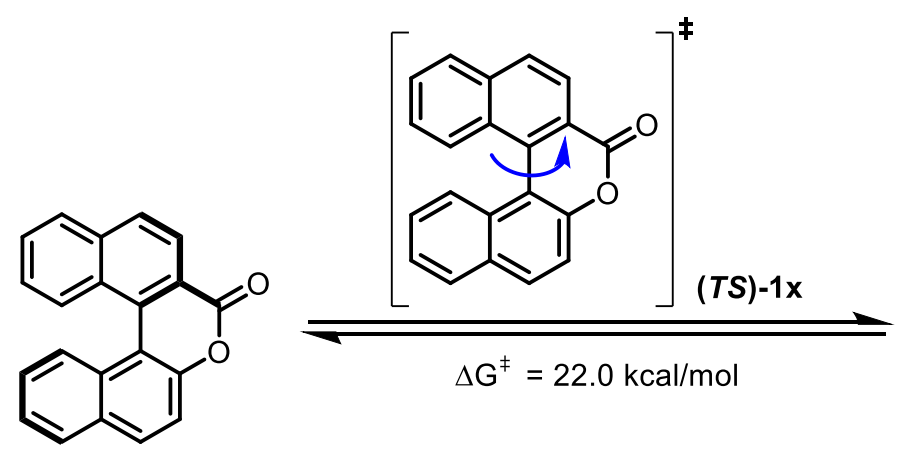

$(R)-1 x$<smiles>O=c1oc2ccc3ccccc3c2c2c1ccc1ccccc12</smiles>

(S)-1x

5. B3-LYP and M11-L absolute calculation energies, enthalpies, and free energies

\begin{tabular}{cccccc}
\hline Geometry & $E_{(\text {elec-B3-LYP) }}{ }^{\mathrm{a}}$ & $\mathrm{H}_{(\text {(corr-B3-LYP) }}{ }^{\mathrm{b}}$ & $\mathrm{G}_{(\text {corr-B3-LYP) }}{ }^{\mathrm{c}}$ & $\mathrm{E}_{(\text {solv-M06) }}{ }^{\mathrm{d}}$ & $\mathrm{IF}^{\mathrm{e}}$ \\
\hline $\boldsymbol{( R ) - 1 a}$ & -882.919865 & 0.294704 & 0.233910 & -882.513905 & \\
$(\boldsymbol{S})-1 \mathbf{a}$ & -882.919865 & 0.294704 & 0.233911 & -882.513903 & \\
$(\boldsymbol{T S})-1 \mathbf{a}$ & -882.892445 & 0.294504 & 0.235023 & -882.485648 & -110.12 \\
$(\boldsymbol{R})-\mathbf{1 x}$ & -957.926640 & 0.285285 & 0.226488 & -957.485611 & \\
$(\boldsymbol{S})-\mathbf{1 x}$ & -957.926640 & 0.285285 & 0.226487 & -957.485612 & \\
$(\boldsymbol{T S})-1 \mathbf{x}$ & -957.890316 & 0.283930 & 0.226192 & -957.450277 & -129.96 \\
\hline
\end{tabular}

\footnotetext{
${ }^{\mathrm{a}}$ The electronic energy calculated by B3-LYP in gas phase. ${ }^{\mathrm{b}}$ The thermal correction to enthalpy calculated by B3-LYP in gas phase. ${ }^{\mathrm{c}}$ The thermal correction to Gibbs free energy calculated by B3-LYP in gas phase. ${ }^{\mathrm{d}}$ The electronic energy calculated by M06 in THF solvent. ${ }^{\mathrm{e}}$ The B3-LYP calculated imaginary frequencies for the transition states.
}

\section{B3-LYP geometries for all the optimized compounds and transition states} (R)-1a

$$
-4.01647900 \quad-1.97066200 \quad-0.50369500
$$




\begin{tabular}{|c|c|c|c|}
\hline $\mathrm{C}$ & -4.11032400 & -0.67352700 & -0.05115200 \\
\hline $\mathrm{C}$ & -2.95176600 & 0.13118200 & 0.11068800 \\
\hline $\mathrm{C}$ & -1.65803900 & -0.44804400 & -0.11626600 \\
\hline $\mathrm{C}$ & -1.60644900 & -1.76674200 & -0.64309400 \\
\hline $\mathrm{C}$ & -2.75232700 & -2.50808300 & -0.83676300 \\
\hline $\mathrm{H}$ & -4.04583800 & 1.93908400 & 0.59950600 \\
\hline $\mathrm{H}$ & -4.91299400 & -2.56911600 & -0.64003600 \\
\hline $\mathrm{H}$ & -5.08136000 & -0.23021700 & 0.15521500 \\
\hline $\mathrm{C}$ & -3.06111300 & 1.51710200 & 0.41655000 \\
\hline $\mathrm{C}$ & -0.48202300 & 0.36593300 & 0.07898900 \\
\hline $\mathrm{H}$ & -0.64785400 & -2.17412700 & -0.94014100 \\
\hline $\mathrm{H}$ & -2.68448900 & -3.50632400 & -1.26023000 \\
\hline $\mathrm{C}$ & -0.66676200 & 1.74967500 & 0.18542900 \\
\hline $\mathrm{C}$ & -1.95114100 & 2.31794900 & 0.38586200 \\
\hline $\mathrm{H}$ & -2.01502700 & 3.39370200 & 0.50731500 \\
\hline $\mathrm{C}$ & 0.90081700 & -0.13403000 & 0.10732100 \\
\hline $\mathrm{C}$ & 1.35372700 & -1.39973000 & 0.57848000 \\
\hline $\mathrm{C}$ & 2.69840700 & -1.74192700 & 0.41098600 \\
\hline $\mathrm{C}$ & 3.23359200 & 0.42083100 & -0.46138000 \\
\hline $\mathrm{C}$ & 3.64412600 & -0.87489300 & -0.15200800 \\
\hline $\mathrm{H}$ & 3.02990200 & -2.71275100 & 0.77395900 \\
\hline $\mathrm{H}$ & 3.93480700 & 1.17734200 & -0.79956900 \\
\hline $\mathrm{C}$ & 1.90166800 & 0.78292200 & -0.28585000 \\
\hline $\mathrm{C}$ & 0.45282500 & 2.68230600 & -0.02844500 \\
\hline $\mathrm{O}$ & 0.39336900 & 3.88793500 & 0.05144900 \\
\hline $\mathrm{O}$ & 1.62859400 & 2.11553200 & -0.47608900 \\
\hline $\mathrm{C}$ & 0.50233000 & -2.34771400 & 1.40381400 \\
\hline $\mathrm{H}$ & -0.38736100 & -1.86261100 & 1.80838100 \\
\hline $\mathrm{H}$ & 0.16854000 & -3.22795900 & 0.84288300 \\
\hline $\mathrm{H}$ & 1.09881100 & -2.71317400 & 2.24701000 \\
\hline $\mathrm{C}$ & 5.07286000 & -1.31143200 & -0.36692500 \\
\hline $\mathrm{H}$ & 5.20348400 & -1.74692400 & -1.36673200 \\
\hline $\mathrm{H}$ & 5.76712600 & -0.46856400 & -0.28655000 \\
\hline $\mathrm{H}$ & 5.37298200 & -2.07278400 & 0.36086700 \\
\hline
\end{tabular}


(S)-1a

\begin{tabular}{|c|c|c|c|}
\hline $\mathrm{C}$ & 4.01649600 & -1.97073500 & -0.50370600 \\
\hline $\mathrm{C}$ & 4.11038000 & -0.67350100 & -0.05148100 \\
\hline $\mathrm{C}$ & 2.95183800 & 0.13120700 & 0.11041900 \\
\hline $\mathrm{C}$ & 1.65807800 & -0.44808800 & -0.11621900 \\
\hline $\mathrm{C}$ & 1.60643800 & -1.76694600 & -0.64269500 \\
\hline $\mathrm{C}$ & 2.75230600 & -2.50829100 & -0.83637800 \\
\hline $\mathrm{H}$ & 4.04597600 & 1.93916800 & 0.59879300 \\
\hline $\mathrm{H}$ & 4.91300300 & -2.56919000 & -0.64006900 \\
\hline $\mathrm{H}$ & 5.08143400 & -0.23012300 & 0.15462900 \\
\hline $\mathrm{C}$ & 3.06122200 & 1.51715900 & 0.41609300 \\
\hline $\mathrm{C}$ & 0.48205100 & 0.36593300 & 0.07906700 \\
\hline $\mathrm{H}$ & 0.64779000 & -2.17447500 & -0.93939400 \\
\hline $\mathrm{H}$ & 2.68441300 & -3.50665100 & -1.25954700 \\
\hline $\mathrm{C}$ & 0.66682000 & 1.74969600 & 0.18541700 \\
\hline $\mathrm{C}$ & 1.95124100 & 2.31798500 & 0.38560000 \\
\hline $\mathrm{H}$ & 2.01512900 & 3.39374600 & 0.50700400 \\
\hline $\mathrm{C}$ & -0.90083700 & -0.13400500 & 0.10742500 \\
\hline $\mathrm{C}$ & -1.35382300 & -1.39969400 & 0.57854700 \\
\hline $\mathrm{C}$ & -2.69848500 & -1.74191700 & 0.41086000 \\
\hline $\mathrm{C}$ & -3.23355300 & 0.42086400 & -0.46153700 \\
\hline $\mathrm{C}$ & -3.64410000 & -0.87488500 & -0.15224400 \\
\hline $\mathrm{H}$ & -3.02997700 & -2.71275500 & 0.77378800 \\
\hline $\mathrm{H}$ & -3.93476800 & 1.17734700 & -0.79978700 \\
\hline $\mathrm{C}$ & -1.90166100 & 0.78299900 & -0.28585300 \\
\hline $\mathrm{C}$ & -0.45280600 & 2.68233000 & -0.02823300 \\
\hline $\mathrm{O}$ & -0.39342500 & 3.88793400 & 0.05198300 \\
\hline $\mathrm{O}$ & -1.62853400 & 2.11558800 & -0.47608900 \\
\hline $\mathrm{C}$ & -0.50258200 & -2.34765800 & 1.40408900 \\
\hline $\mathrm{H}$ & -0.16996300 & -3.22861900 & 0.84356700 \\
\hline $\mathrm{H}$ & 0.38777100 & -1.86299200 & 1.80769600 \\
\hline $\mathrm{H}$ & -1.09880900 & -2.71196500 & 2.24796800 \\
\hline $\mathrm{C}$ & -5.07282900 & -1.31138200 & -0.36724200 \\
\hline $\mathrm{H}$ & -5.76699500 & -0.46837900 & -0.28747400 \\
\hline
\end{tabular}


$\mathrm{H}$

$\mathrm{H}$

(TS)-1a

C

C

C

C

C

C

$\mathrm{H}$

$\mathrm{H}$

$\mathrm{H}$

C

C

$\mathrm{H}$

$\mathrm{H}$

C

C

$\mathrm{H}$

C

$\mathrm{O}$

$\mathrm{O}$

C

C

C

C

C

C

$\mathrm{H}$

$\mathrm{H}$

C

$\mathrm{H}$

$\mathrm{H}$

C

C

C

C

C

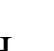

H

C

H

H

$\mathrm{C}$

H

C

O

O

C

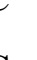

C

$\mathrm{C}$

$\mathrm{C}$

$\mathrm{H}$

H

C

H

H
$-5.20326500$

$-1.74744900$

$-1.36681400$

$\begin{array}{lll}-5.37323100 & -2.07228400 & 0.36091300\end{array}$

\begin{tabular}{rrr}
-4.29122800 & -1.79900600 & 0.34757900 \\
-4.18573100 & -0.44603600 & 0.56227700 \\
-2.97356900 & 0.25589900 & 0.32846800 \\
-1.77438200 & -0.44569500 & -0.07007800 \\
-1.97259100 & -1.82063900 & -0.35426800 \\
-3.16828500 & -2.48075100 & -0.15539100 \\
-3.91029900 & 2.17075400 & 0.71977900 \\
-5.22749700 & -2.32108500 & 0.52304300 \\
-5.04640900 & 0.13022800 & 0.89232600 \\
-2.98963700 & 1.66810800 & 0.43644600 \\
-0.50368700 & 0.28732000 & -0.19202300 \\
-1.18296400 & -2.37886300 & -0.79987000 \\
-3.23825600 & -3.53522900 & -0.40885800 \\
-0.64706800 & 1.69236700 & -0.14537200 \\
-1.86500100 & 2.36734700 & 0.13111800 \\
-1.83932600 & 3.45033100 & 0.13504200 \\
0.46591900 & 2.63337700 & -0.32688400 \\
0.36587500 & 3.81964700 & -0.55371800 \\
1.70550300 & 2.10940300 & -0.16346500 \\
0.92259100 & -0.23668400 & -0.21196600 \\
1.49021800 & -1.57183400 & -0.26108100 \\
1.92644600 & 0.76293100 & -0.03812700 \\
2.84074900 & -1.77180900 & 0.04835500 \\
3.27123500 & 0.53003800 & 0.25593800 \\
3.75317300 & -0.75896000 & 0.35332700 \\
3.90544100 & 1.39960900 & 0.39471200 \\
3.21397600 & -2.79185000 & 0.02157300 \\
0.84725100 & -2.87360600 & -0.71903500 \\
\hline .18009800 & -3.31992800 & 0.02334400 \\
\hline
\end{tabular}


H

C

H

H

H

(R)-1x

C

C

C

C

C

C

$\mathrm{H}$

H

$\mathrm{H}$

C

C

H

$\mathrm{H}$

C

C

$\mathrm{H}$

C

O

$\mathrm{O}$

C

C

C

C

C

C

C

H

.

.

(1)

C

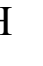

H

C

C

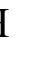

C

C

H

C

O

O

C

C

C

C

C

$\mathrm{H}$ $\begin{array}{lll}1.63641800 & -3.60561200 & -0.90680100\end{array}$

$\begin{array}{lll}5.18070200 & -1.06359500 & 0.72855900\end{array}$

$\begin{array}{lll}5.82645700 & -0.19117300 & 0.58846800\end{array}$

$5.25362100 \quad-1.36254000 \quad 1.78262400$

$\begin{array}{lll}5.58448600 & -1.88828900 & 0.13056200\end{array}$

\begin{tabular}{rrr}
3.04801400 & -3.10530400 & 0.62740200 \\
3.62366200 & -2.00660500 & 0.03048700 \\
2.88779100 & -0.80785100 & -0.16320600 \\
1.49757300 & -0.76754200 & 0.19358100 \\
0.95607700 & -1.90264300 & 0.85832200 \\
1.70908800 & -3.03720800 & 1.07334600 \\
4.58326100 & 0.31527900 & -0.91676600 \\
3.62759300 & -4.01083600 & 0.78410800 \\
4.66733600 & -2.02657800 & -0.27324500 \\
3.53459100 & 0.36872700 & -0.63651000 \\
0.75238600 & 0.45317500 & -0.02977900 \\
-0.06068200 & -1.86517700 & 1.22717800 \\
1.27053700 & -3.88112400 & 1.59842600 \\
1.48908800 & 1.61210800 & -0.31373700 \\
\hline 2.86685600 & 1.56243100 & -0.64893300 \\
3.35885300 & 2.49447400 & -0.90417200 \\
0.89340000 & 2.94859800 & -0.16549900 \\
1.43613300 & 4.00209400 & -0.40583700 \\
-0.36159000 & 2.98055400 & 0.41582800 \\
-0.70566700 & 0.60373800 & 0.09075900 \\
-1.71335600 & -0.40247600 & -0.18142300 \\
-1.17014800 & 1.87712800 & 0.42043800 \\
-1.44780900 & -1.60463000 & -0.89299300 \\
-3.07845900 & -0.14020300 & 0.17641700 \\
-2.51206600 & 2.13926300 & 0.77715600 \\
-2.44281000 & -2.52511300 & -1.15009000 \\
-0.44873700 & -1.79211000 & -1.26517600
\end{tabular}




$\begin{array}{cccc}\mathrm{C} & -4.07258800 & -1.12395400 & -0.06709300 \\ \mathrm{C} & -3.43615800 & 1.13163100 & 0.70460100 \\ \mathrm{H} & -2.77124100 & 3.14499100 & 1.09039000 \\ \mathrm{C} & -3.76557500 & -2.30234800 & -0.70880300 \\ \mathrm{H} & -2.20487500 & -3.42588000 & -1.70938400 \\ \mathrm{H} & -5.09441200 & -0.91085800 & 0.23774000 \\ \mathrm{H} & -4.46979400 & 1.31269200 & 0.98715800 \\ \mathrm{H} & -4.53751900 & -3.04158200 & -0.90284200\end{array}$

(S)-1x

\begin{tabular}{|c|c|c|c|}
\hline $\mathrm{C}$ & 3.04800300 & -3.10537400 & -0.62736900 \\
\hline $\mathrm{C}$ & 3.62363800 & -2.00667900 & -0.03044600 \\
\hline $\mathrm{C}$ & 2.88776700 & -0.80792900 & 0.16325200 \\
\hline $\mathrm{C}$ & 1.49754200 & -0.76762100 & -0.19354400 \\
\hline $\mathrm{C}$ & 0.95605800 & -1.90272700 & -0.85828600 \\
\hline $\mathrm{C}$ & 1.70907900 & -3.03728200 & -1.07331000 \\
\hline $\mathrm{H}$ & 4.58326100 & 0.31518700 & 0.91674600 \\
\hline $\mathrm{H}$ & 3.62759200 & -4.01089500 & -0.78409900 \\
\hline $\mathrm{H}$ & 4.66731900 & -2.02663600 & 0.27326100 \\
\hline $\mathrm{C}$ & 3.53459600 & 0.36866200 & 0.63648400 \\
\hline $\mathrm{C}$ & 0.75242600 & 0.45313000 & 0.02980800 \\
\hline $\mathrm{H}$ & -0.06068700 & -1.86526300 & -1.22717300 \\
\hline $\mathrm{H}$ & 1.27053900 & -3.88119300 & -1.59840800 \\
\hline $\mathrm{C}$ & 1.48915000 & 1.61209400 & 0.31365900 \\
\hline $\mathrm{C}$ & 2.86692200 & 1.56240700 & 0.64882000 \\
\hline $\mathrm{H}$ & 3.35896000 & 2.49447800 & 0.90386100 \\
\hline $\mathrm{C}$ & 0.89341900 & 2.94861600 & 0.16536000 \\
\hline $\mathrm{O}$ & 1.43628200 & 4.00203900 & 0.40588400 \\
\hline $\mathrm{O}$ & -0.36145600 & 2.98060500 & -0.41572900 \\
\hline $\mathrm{C}$ & -0.70563000 & 0.60370900 & -0.09071200 \\
\hline $\mathrm{C}$ & -1.71335700 & -0.40248700 & 0.18142900 \\
\hline $\mathrm{C}$ & -1.17007200 & 1.87712200 & -0.42038300 \\
\hline $\mathrm{C}$ & -1.44796800 & -1.60463900 & 0.89307800 \\
\hline $\mathrm{C}$ & -3.07844400 & -0.14012400 & -0.17648500 \\
\hline
\end{tabular}




$\begin{array}{lrrr}\mathrm{C} & -2.51198400 & 2.13935300 & -0.77708400 \\ \mathrm{C} & -2.44304600 & -2.52505700 & 1.15010500 \\ \mathrm{H} & -0.44898600 & -1.79214500 & 1.26542300 \\ \mathrm{C} & -4.07264700 & -1.12382100 & 0.06695900 \\ \mathrm{C} & -3.43608700 & 1.13173800 & -0.70463600 \\ \mathrm{H} & -2.77112100 & 3.14512900 & -1.09018700 \\ \mathrm{C} & -3.76576200 & -2.30222300 & 0.70869900 \\ \mathrm{H} & -2.20521500 & -3.42580300 & 1.70945700 \\ \mathrm{H} & -5.09443000 & -0.91065200 & -0.23793400 \\ \mathrm{H} & -4.46969200 & 1.31283400 & -0.98724100 \\ \mathrm{H} & -4.53776200 & -3.04140400 & 0.90267300\end{array}$

\section{(TS)-1x}

\begin{tabular}{|c|c|c|c|}
\hline $\mathrm{C}$ & 3.15972100 & -3.17438400 & 0.30795100 \\
\hline $\mathrm{C}$ & 3.58327800 & -1.94789200 & 0.76371500 \\
\hline $\mathrm{C}$ & 2.85549900 & -0.76053400 & 0.48757700 \\
\hline $\mathrm{C}$ & 1.57014500 & -0.82387800 & -0.16722600 \\
\hline $\mathrm{C}$ & 1.25935500 & -2.08897700 & -0.72978000 \\
\hline $\mathrm{C}$ & 2.01181600 & -3.22476300 & -0.50421200 \\
\hline $\mathrm{H}$ & 4.44573900 & 0.51071800 & 1.23528700 \\
\hline $\mathrm{H}$ & 3.73388500 & -4.07334000 & 0.51305900 \\
\hline $\mathrm{H}$ & 4.52024800 & -1.85327000 & 1.30675300 \\
\hline $\mathrm{C}$ & 3.46655900 & 0.49045700 & 0.76500700 \\
\hline $\mathrm{C}$ & 0.76835100 & 0.40401800 & -0.27735700 \\
\hline $\mathrm{H}$ & 0.46910000 & -2.16437300 & -1.44960100 \\
\hline $\mathrm{H}$ & 1.72270300 & -4.15613700 & -0.98304100 \\
\hline $\mathrm{C}$ & 1.52205900 & 1.59184500 & -0.13599700 \\
\hline $\mathrm{C}$ & 2.85598800 & 1.63308000 & 0.34540400 \\
\hline $\mathrm{H}$ & 3.32720500 & 2.60557500 & 0.42765400 \\
\hline $\mathrm{C}$ & 0.96777900 & 2.91890200 & -0.41741000 \\
\hline $\mathrm{O}$ & 1.59868300 & 3.92431700 & -0.65508700 \\
\hline $\mathrm{O}$ & -0.39435000 & 3.00092300 & -0.35457200 \\
\hline $\mathrm{C}$ & -0.72855600 & 0.57757900 & -0.29137400 \\
\hline $\mathrm{C}$ & -1.81694600 & -0.41241600 & -0.18719900 \\
\hline
\end{tabular}




$\begin{array}{lrrr}\mathrm{C} & -1.17131400 & 1.90165900 & -0.12516900 \\ \mathrm{C} & -1.82332200 & -1.71538100 & -0.74699200 \\ \mathrm{C} & -3.05897100 & -0.03782500 & 0.44576000 \\ \mathrm{C} & -2.44231200 & 2.28168800 & 0.36587500 \\ \mathrm{C} & -2.84141000 & -2.62798100 & -0.54069100 \\ \mathrm{H} & -1.06211800 & -1.99376800 & -1.44751500 \\ \mathrm{C} & -4.07182400 & -0.99987100 & 0.69151100 \\ \mathrm{C} & -3.33562400 & 1.32408500 & 0.73684900 \\ \mathrm{H} & -2.62749500 & 3.34208500 & 0.49687000 \\ \mathrm{C} & -3.96140900 & -2.29490700 & 0.24009800 \\ \mathrm{H} & -2.77877500 & -3.60324900 & -1.01577600 \\ \mathrm{H} & -4.96690800 & -0.67102100 & 1.21387000 \\ \mathrm{H} & -4.28374400 & 1.58860200 & 1.19642200 \\ \mathrm{H} & -4.74768200 & -3.02077500 & 0.42525800\end{array}$




\section{Gram-scale synthesis of 3a}
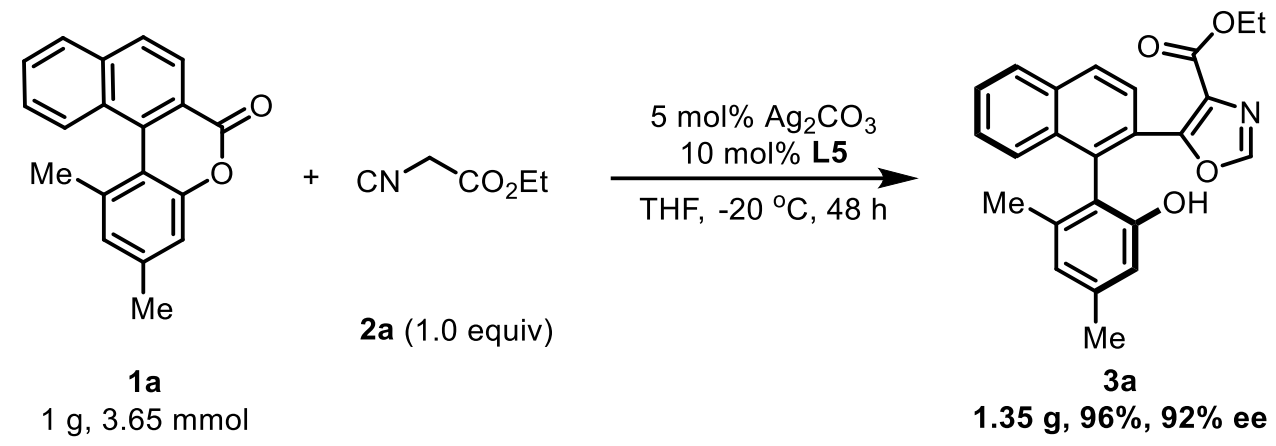

To a $100 \mathrm{~mL}$ Round-bottom flask charged with $\mathbf{L 5}(212.1 \mathrm{mg}, 0.365 \mathrm{mmol})$ and $\mathrm{Ag}_{2} \mathrm{CO}_{3}(50.4 \mathrm{mg}, 0.183 \mathrm{mmol})$ was added anhydrous THF $(36.0 \mathrm{~mL})$. The mixture was stirred at ambient temperature for $5 \mathrm{~min}$, then biaryl lactone $1 \mathrm{a}(1 \mathrm{~g}, 3.65 \mathrm{mmol})$ was added. After the mixture was cooled to $-20^{\circ} \mathrm{C}$, ethyl isocyanoacetate $2 \mathrm{a}$ (412.5 $\mathrm{mg}, 3.65 \mathrm{mmol}$ ) was added in one portion. The reaction mixture was stirred at $-20{ }^{\circ} \mathrm{C}$ for $48 \mathrm{~h}$, concentrated and purified by flash chromatography (PE/EtOAc 2:1) to afford the product 3a.

\section{Synthetic transformations}<smiles>CCOC(=O)c1ncoc1-c1ccc2ccccc2c1-c1c(C)cc(C)cc1O</smiles>

3a: $92 \%$ ee<smiles>Cc1cc(O)c(-c2c(-c3ocnc3CO)ccc3ccccc23)c(O)c1</smiles>

5: $81 \%, 92 \%$ ee

$\mathrm{LiAlH}_{4}(15.2 \mathrm{mg}, 0.40 \mathrm{mmol})$ was added in several portions to the solution of $\mathbf{3 a}$ (39 $\mathrm{mg}, 0.10 \mathrm{mmol}, 92 \%$ ee $)$ in anhydrous THF $(2.0 \mathrm{~mL})$ at $-20{ }^{\circ} \mathrm{C}$. The reaction mixture was stirred at $-20{ }^{\circ} \mathrm{C}$ for $1.5 \mathrm{~h}$. Upon completion, the reaction was quenched with water, and the solution was extracted with ethyl acetate. The combined organic layers were washed with brine, dried over anhydrous $\mathrm{Na}_{2} \mathrm{SO}_{4}$, then concentrated and purified by flash chromatography (PE/EtOAc 1:1 to EtOAc) to afford $28.3 \mathrm{mg}$ of $\mathbf{5}$. 


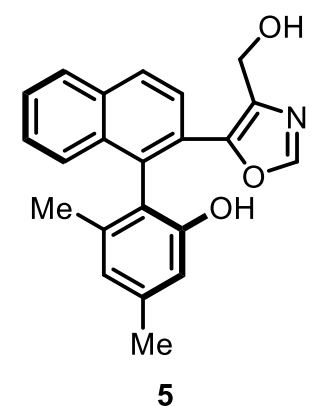

White solid, 81\% yield. MP: $163-165{ }^{\circ} \mathrm{C} ;{ }^{1} \mathbf{H}$ NMR (500 MHz, DMSO): $\delta 8.96$ (s, 1H), $8.11(\mathrm{~s}, 1 \mathrm{H}), 8.06-7.97(\mathrm{~m}, 2 \mathrm{H}), 7.69(\mathrm{~d}, J=8.5 \mathrm{~Hz}, 1 \mathrm{H}), 7.60-7.53(\mathrm{~m}, 1 \mathrm{H})$, 7.48-7.40 (m, 1H), $7.33(\mathrm{~d}, J=8.2 \mathrm{~Hz}, 1 \mathrm{H}), 6.55(\mathrm{~s}, 1 \mathrm{H}), 6.52(\mathrm{~s}, 1 \mathrm{H}), 4.99(\mathrm{t}, J=5.6$ $\mathrm{Hz}, 1 \mathrm{H}), 4.28(\mathrm{dd}, J=12.7,5.7 \mathrm{~Hz}, 1 \mathrm{H}), 4.13(\mathrm{dd}, J=12.7,5.6 \mathrm{~Hz}, 1 \mathrm{H}), 2.23(\mathrm{~s}, 3 \mathrm{H})$, $1.63(\mathrm{~s}, 3 \mathrm{H}) ;{ }^{13} \mathrm{C}$ NMR (126 MHz, DMSO): $\delta$ 155.0, 150.7, 147.1, 137.4, 137.0, $136.3,136.0,133.3,132.1,128.0,127.3,126.7,126.6,126.0,125.3,121.5,121.3$, 113.3, 54.7, 21.0, 19.4; HRMS (ESI): $\mathrm{m} / \mathrm{z}$ calcd. for $\left[\mathrm{C}_{22} \mathrm{H}_{19} \mathrm{NNaO}_{3}, \mathrm{M}+\mathrm{Na}\right]^{+}$: 368.1257; found: 368.1257 .

Optical Rotation: $[\alpha]_{D}^{25}=+52.0(\mathrm{c}=0.1$, THF). The absolute configuration of 5 was assigned by analogy to 3a. $92 \%$ ee (HPLC condition: Chiralpak IB N-5 column, $n$-hexane $/ i-\operatorname{PrOH}=80: 20$, flow rate $=1 \mathrm{ml} / \mathrm{min}$, wavelength $=254 \mathrm{~nm}, \mathrm{t}_{\mathrm{R}}=6.0 \mathrm{~min}$ for minor isomer, $t_{R}=7.5$ min for major isomer).

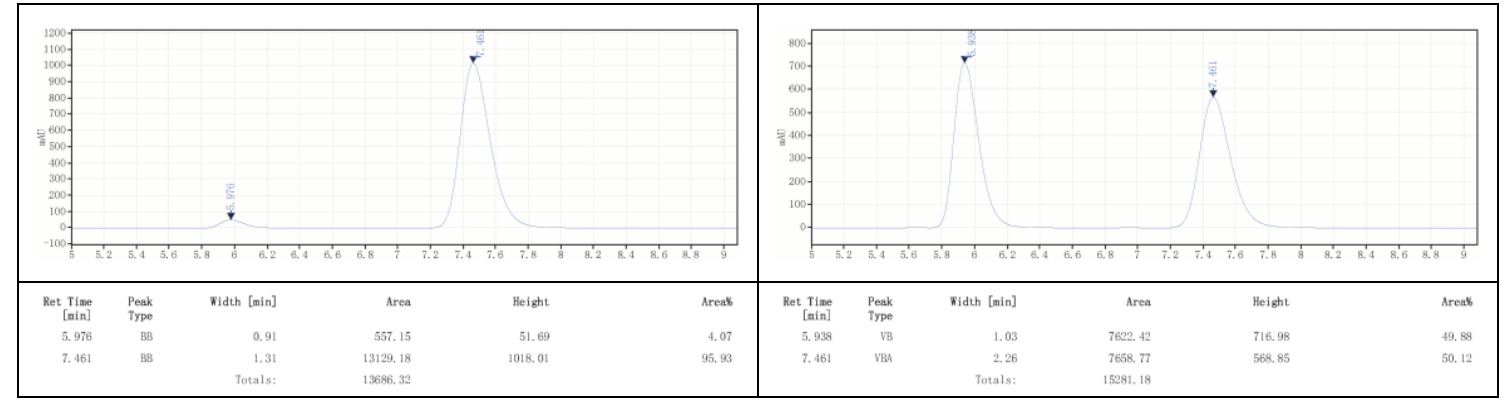

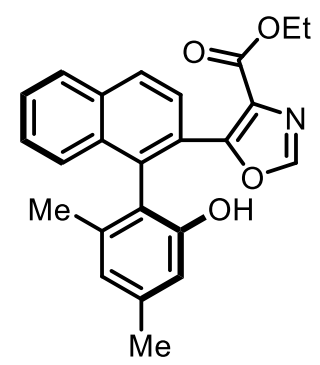

3a: $92 \%$ ee

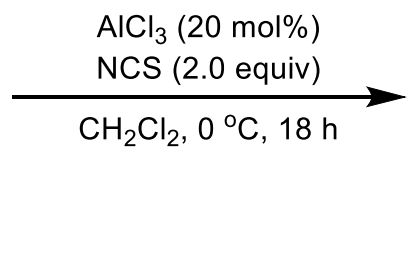

$\mathrm{AlCl}_{3}(20 \mathrm{~mol} \%)$

$\mathrm{CH}_{2} \mathrm{Cl}_{2}, 0^{\circ} \mathrm{C}, 18 \mathrm{~h}$ 
NCS $(13.3 \mathrm{mg}, 0.10 \mathrm{mmol})$ and $\mathrm{AlCl}_{3}(1.3 \mathrm{mg}, 0.01 \mathrm{mmol})$ was added to the mixture of $3 \mathbf{a}(19.5 \mathrm{mg}, 0.05 \mathrm{mmol}, 92 \%$ ee $)$ in anhydrous $\mathrm{CH}_{2} \mathrm{Cl}_{2}(1.0 \mathrm{~mL})$ at $0{ }^{\circ} \mathrm{C}$. The reaction mixture was stirred at $0{ }^{\circ} \mathrm{C}$ for $18 \mathrm{~h}$, then concentrated and purified by flash chromatography (PE/EtOAc 2:1) to afford $18.1 \mathrm{mg}$ of $\mathbf{6}$.

\section{Ethyl}

(S)-5-(1-(3,5-dichloro-2-hydroxy-4,6-dimethylphenyl)naphthalen-2-yl)oxazole-4-c arboxylate (6)<smiles>CCOC(=O)c1ncoc1-c1ccc2ccccc2c1-c1c(C)c(Cl)c([N+](=O)[O-])c(Cl)c1O</smiles>

White solid, 79\% yield. MP: 200-202 ${ }^{\circ} \mathrm{C}$; ${ }^{1} \mathbf{H}$ NMR (500 MHz, $\left.\mathrm{CDCl}_{3}\right): \delta 8.02$ (d, $J=$ $8.5 \mathrm{~Hz}, 1 \mathrm{H}), 7.97(\mathrm{~d}, J=8.2 \mathrm{~Hz}, 1 \mathrm{H}), 7.70(\mathrm{~s}, 1 \mathrm{H}), 7.67$ (d, $J=8.5 \mathrm{~Hz}, 1 \mathrm{H}), 7.62-7.57$ (m, 1H), 7.49-7.43 (m, 1H), 7.38 (d, $J=8.5 \mathrm{~Hz}, 1 \mathrm{H}), 5.45$ (br s, 1H), 4.35-4.23 (m, 2H), $2.53(\mathrm{~s}, 3 \mathrm{H}), 1.89(\mathrm{~s}, 3 \mathrm{H}), 1.26(\mathrm{t}, J=7.1 \mathrm{~Hz}, 3 \mathrm{H}) ;{ }^{13} \mathbf{C ~ N M R}\left(126 \mathrm{MHz}, \mathrm{CDCl}_{3}\right)$ : $\delta 161.6,155.8,150.2,148.1,135.2,134.9,134.8,134.5,131.9,128.7,128.6,128.5$, 127.9, 127.9, 127.6, 127.1, 126.0, 125.5, 123.4, 119.3, 61.5, 18.8, 18.3, 14.2; HRMS (ESI): m/z calcd. for $\left[\mathrm{C}_{24} \mathrm{H}_{19} \mathrm{Cl}_{2} \mathrm{NNaO}_{4}, \mathrm{M}+\mathrm{Na}\right]^{+}:$478.0583; found: 478.0585 .

Optical Rotation: $[\alpha]^{25}=+52.2(\mathrm{c}=0.3$, THF). The absolute configuration of 6 was assigned by analogy to 3a. 92\% ee (HPLC condition: Chiralpak IC column, $n$-hexane $/ i-\mathrm{PrOH}=90: 10$, flow rate $=1 \mathrm{ml} / \mathrm{min}$, wavelength $=254 \mathrm{~nm}, \mathrm{t}_{\mathrm{R}}=15.5 \mathrm{~min}$ for major isomer, $t_{\mathrm{R}}=25.1 \mathrm{~min}$ for minor isomer). 


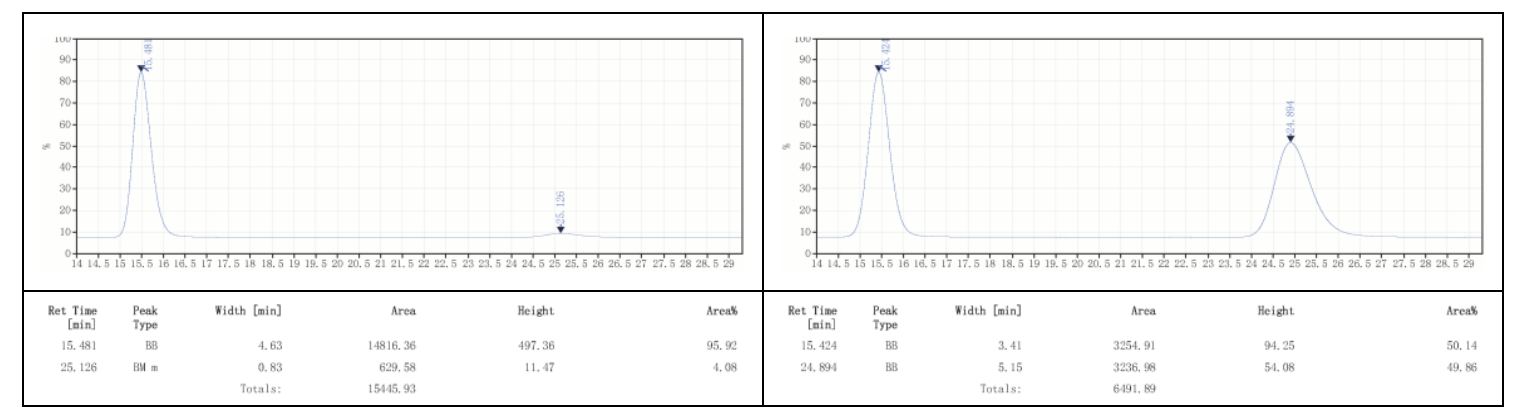

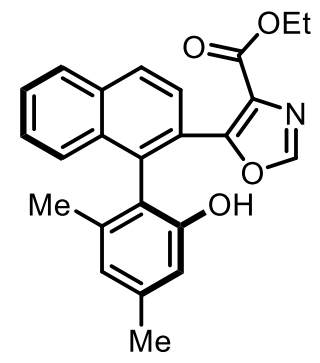

3a: $92 \%$ ee
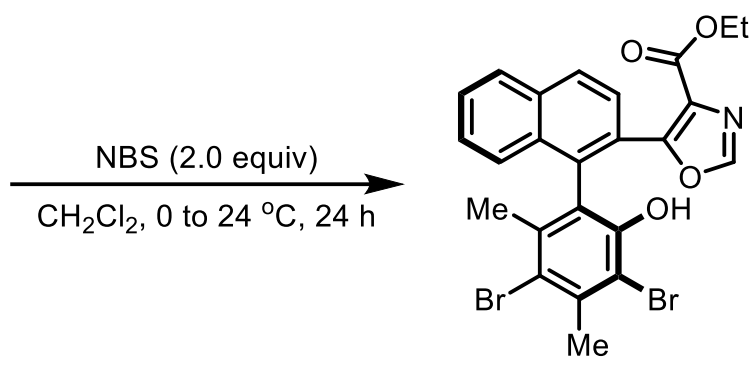

7: $93 \%, 92 \%$ ee

NBS (17.8 mg, $0.10 \mathrm{mmol})$ was added to the mixture of $\mathbf{3 a}(19.5 \mathrm{mg}, 0.05 \mathrm{mmol}, 92 \%$ ee) in anhydrous $\mathrm{CH}_{2} \mathrm{Cl}_{2}(1.0 \mathrm{~mL})$ at $0{ }^{\circ} \mathrm{C}$. The reaction mixture was stirred for $20 \mathrm{~h}$ at $0{ }^{\circ} \mathrm{C}$ and another $4 \mathrm{~h}$ at ambient temperature, then concentrated and purified by flash chromatography (PE/EtOAc 2:1) to afford $25.3 \mathrm{mg}$ of 7.

\section{Ethyl}

(S)-5-(1-(3,5-dibromo-2-hydroxy-4,6-dimethylphenyl)naphthalen-2-yl)oxazole-4carboxylate (7)<smiles>CCOC(=O)c1ncoc1-c1ccc2ccccc2c1-c1c(C)c(Br)c([N+](=O)[O-])c(Br)c1O</smiles>

White solid, 93\% yield. MP: $243-245{ }^{\circ} \mathrm{C} ;{ }^{1} \mathbf{H}$ NMR (500 MHz, $\left.\mathrm{CDCl}_{3}\right): \delta 8.01$ (d, $J=$ $8.5 \mathrm{~Hz}, 1 \mathrm{H}), 7.96(\mathrm{~d}, J=8.2 \mathrm{~Hz}, 1 \mathrm{H}), 7.70(\mathrm{~s}, 1 \mathrm{H}), 7.67(\mathrm{~d}, J=8.5 \mathrm{~Hz}, 1 \mathrm{H}), 7.62-7.57$ (m, 1H), 7.48-7.43 (m, 1H), $7.38(\mathrm{~d}, J=8.3 \mathrm{~Hz}, 1 \mathrm{H}), 5.52$ (br s, 1H), 4.34-4.24 (m, 2H), $2.67(\mathrm{~s}, 3 \mathrm{H}), 1.94(\mathrm{~s}, 3 \mathrm{H}), 1.26(\mathrm{t}, J=7.1 \mathrm{~Hz}, 3 \mathrm{H}) ;{ }^{13} \mathbf{C ~ N M R}\left(126 \mathrm{MHz}, \mathrm{CDCl}_{3}\right)$ : $\delta 161.6,155.7,150.1,149.6,138.1,137.7,135.2,134.5,131.8,128.7,128.6,128.5$, 
127.9, 127.9, 127.6, 126.0, 125.4, 123.2, 119.0, 111.0, 61.5, 25.4, 21.8, 14.2; HRMS (ESI): $\mathrm{m} / \mathrm{z}$ calcd. for $\left[\mathrm{C}_{24} \mathrm{H}_{19} \mathrm{Br}_{2} \mathrm{NNaO}_{4}, \mathrm{M}+\mathrm{Na}\right]^{+}:$565.9573; found: 565.9575 .

Optical Rotation: $[\alpha]^{25}=+47.5(\mathrm{c}=0.3$, THF). The absolute configuration of 7 was assigned by analogy to 3a. $92 \%$ ee (HPLC condition: Chiralpak IC column, $n$-hexane $/ i-\operatorname{PrOH}=90: 10$, flow rate $=1 \mathrm{ml} / \mathrm{min}$, wavelength $=254 \mathrm{~nm}, \mathrm{t}_{\mathrm{R}}=17.3 \mathrm{~min}$ for major isomer, $t_{\mathrm{R}}=27.6 \mathrm{~min}$ for minor isomer).

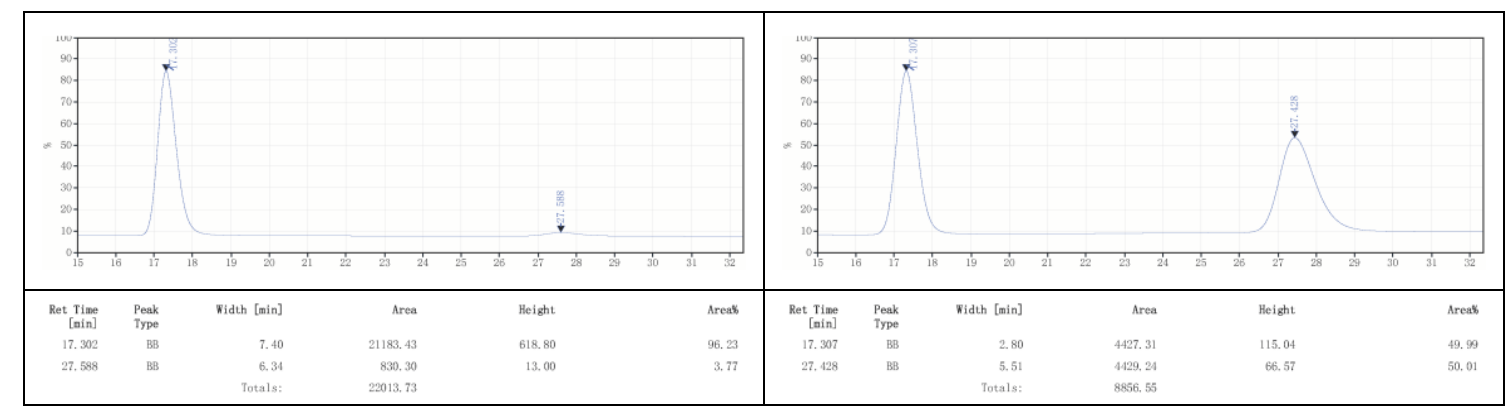

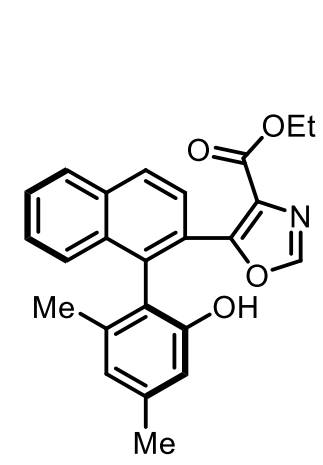

3a: $92 \%$ ee

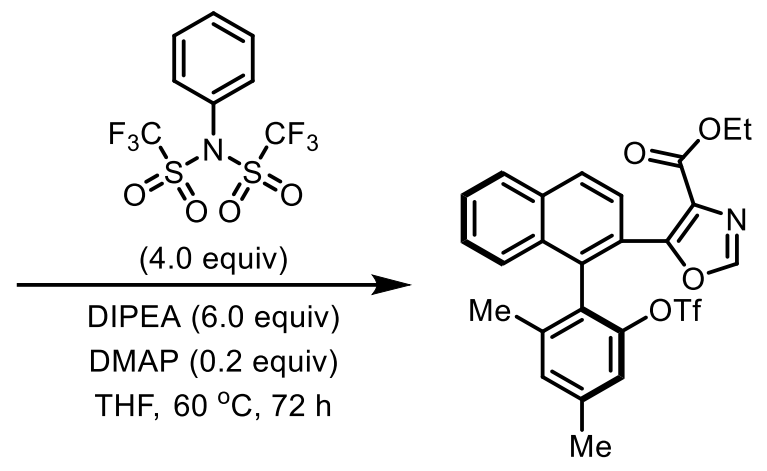

8: $89 \%, 92 \%$ ee

$N$-phenyl-bis(trifluoromethanesulfonimide) $(370 \mathrm{mg}, 1.04 \mathrm{mmol}$ ) was added to the mixture of 3a (100 mg, $0.26 \mathrm{mmol}, 92 \%$ ee) in anhydrous $\mathrm{THF}(20.0 \mathrm{~mL})$ at $24{ }^{\circ} \mathrm{C}$, followed by DIPEA (202 mg, $1.56 \mathrm{mmol})$ and DMAP (6.3 mg, $0.05 \mathrm{mmol})$. The reaction mixture was stirred at $60{ }^{\circ} \mathrm{C}$ for $72 \mathrm{~h}$, then concentrated and purified by flash chromatography (PE/EtOAc 5:1) to afford $120 \mathrm{mg}$ of $\mathbf{8}$.

\section{Ethyl}

(S)-5-(1-(2,4-dimethyl-6-(((trifluoromethyl)sulfonyl)oxy)phenyl)naphthalen-2-yl) oxazole-4-carboxylate (8) 


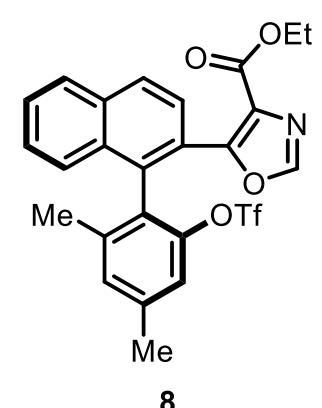

Yellow oil, $89 \%$ yield. ${ }^{1} \mathbf{H}$ NMR $\left(400 \mathrm{MHz}, \mathrm{CDCl}_{3}\right): \delta 8.00(\mathrm{~d}, J=8.6 \mathrm{~Hz}, 1 \mathrm{H}), 7.95$ $(\mathrm{d}, J=8.2 \mathrm{~Hz}, 1 \mathrm{H}), 7.74(\mathrm{~d}, J=8.5 \mathrm{~Hz}, 1 \mathrm{H}), 7.63(\mathrm{~s}, 1 \mathrm{H}), 7.61-7.54(\mathrm{~m}, 1 \mathrm{H})$, 7.49-7.42 (m, 1H), 7.39 (d, $J=8.4 \mathrm{~Hz}, 1 \mathrm{H}), 7.08$ (s, 1H), 6.98 (s, 1H), 4.39-4.16 (m, 2H), 2.39 (s, 3H), $1.92(\mathrm{~s}, 3 \mathrm{H}), 1.24(\mathrm{t}, J=7.1 \mathrm{~Hz}, 3 \mathrm{H}) ;{ }^{13} \mathbf{C ~ N M R}\left(101 \mathrm{MHz}, \mathrm{CDCl}_{3}\right)$ : $\delta 161.2,155.4,150.0,147.7,140.8,140.1,134.3,133.2,131.8,130.4,128.6,128.4$, $128.0,127.7,127.5,127.2,126.2,125.1,119.7,119.0,116.5,61.3,21.3,20.0,14.1$; HRMS (ESI): $\mathrm{m} / \mathrm{z}$ calcd. for $\left[\mathrm{C}_{25} \mathrm{H}_{20} \mathrm{~F}_{3} \mathrm{NNaO}_{6} \mathrm{~S}, \mathrm{M}+\mathrm{Na}\right]^{+}:$542.0856; found: 542.0856.

Optical Rotation: $[\alpha]^{25}{ }_{\mathrm{D}}=+46.0(\mathrm{c}=0.1$, THF). The absolute configuration of $\mathbf{8}$ was assigned by analogy to 3a. 92\% ee (HPLC condition: Chiralpak IB N-5 column, $n$-hexane $/ i-\operatorname{PrOH}=95: 5$, flow rate $=1 \mathrm{ml} / \mathrm{min}$, wavelength $=254 \mathrm{~nm}, \mathrm{t}_{\mathrm{R}}=19.8 \mathrm{~min}$ for major isomer, $t_{R}=21.3 \mathrm{~min}$ for minor isomer).

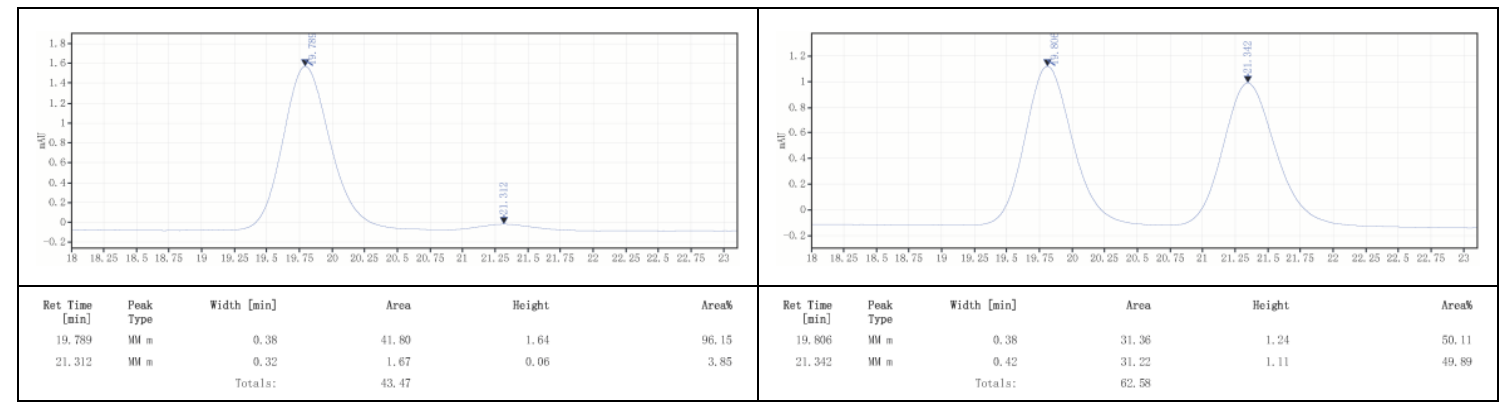

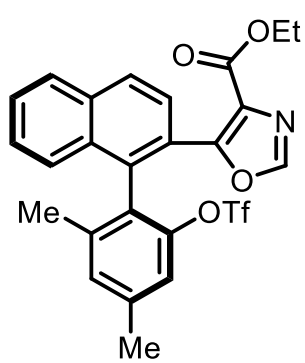

8: $92 \%$ ee

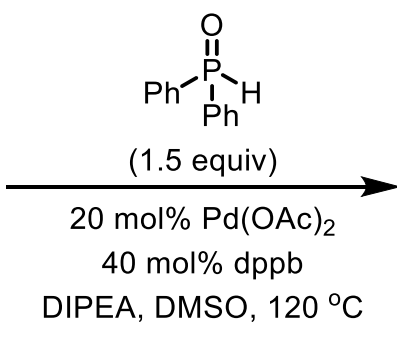
DIPEA, DMSO, $120^{\circ} \mathrm{C}$

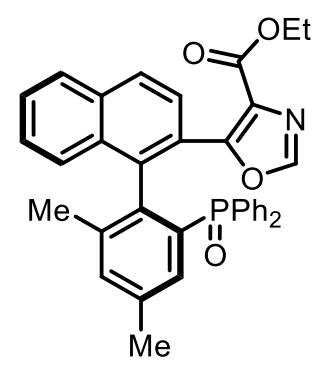

9: $88 \%, 94 \%$ ee 
Diphenylphosphine oxide (89 mg, $0.44 \mathrm{mmol}$ ) was added to the mixture of 8 (150 mg, $0.29 \mathrm{mmol}, 92 \%$ ee $)$ in anhydrous $\mathrm{DMSO}(5.0 \mathrm{~mL})$ at $24{ }^{\circ} \mathrm{C}$, and then $\mathrm{Pd}(\mathrm{OAc})_{2}(13.5$ mg, $0.06 \mathrm{mmol})$, dppb (51.2 mg, $0.12 \mathrm{mmol})$ and DIPEA (187 mg, $1.45 \mathrm{mmol})$ were added. The reaction mixture was stirred at $120^{\circ} \mathrm{C}$ for $48 \mathrm{~h}$, and then extracted with EtOAc/ $\mathrm{H}_{2} \mathrm{O}$. The organic layer was dried, concentrated and purified by flash chromatography (EtOAc) to afford $145 \mathrm{mg}$ of $\mathbf{9}$.

\section{Ethyl}

(S)-5-(1-(2-(diphenylphosphoryl)-4,6-dimethylphenyl)naphthalen-2-yl)oxazole-4carboxylate (9)<smiles>CCOC(=O)c1nc[o+](P)c1-c1ccc2ccccc2c1-c1c(C)cc(C)cc1C</smiles>

White solid, 88\% yield. MP: $156-157{ }^{\circ} \mathrm{C} ;{ }^{1} \mathbf{H}$ NMR (400 MHz, DMSO): $\delta 8.19$ (s, 1H), $7.83(\mathrm{~d}, J=8.6 \mathrm{~Hz}, 2 \mathrm{H}), 7.64(\mathrm{~d}, J=8.6 \mathrm{~Hz}, 1 \mathrm{H}), 7.44-7.29(\mathrm{~m}, 8 \mathrm{H}), 7.26-7.20$ (m, 2H), 7.19-7.11 (m, 3H), $7.05(\mathrm{t}, J=7.6 \mathrm{~Hz}, 1 \mathrm{H}), 6.85(\mathrm{~d}, J=8.4 \mathrm{~Hz}, 1 \mathrm{H})$, 4.29-3.98 (m, 2H), 2.29 (s, 3H), $1.66(\mathrm{~s}, 3 \mathrm{H}), 1.14(\mathrm{t}, J=7.1 \mathrm{~Hz}, 3 \mathrm{H}) ;{ }^{13} \mathbf{C}$ NMR (101 MHz, DMSO): $\delta$ 160.8, 155.3, 150.8, 138.5, 138.4, 138.2, 138.1, 137.4, 137.3, 136.7, $136.5,134.1,134.0,133.7,133.3,133.1,132.7,132.5,131.5,131.3,131.2,131.1$, 131.0, 128.3, 128.1, 128.0, 127.9, 127.8, 127.7, 126.9, 126.7, 125.8, 123.9, 60.4, 20.8, 19.3, 13.9; ${ }^{31} \mathbf{P}$ NMR (162 MHz, DMSO): $\delta$ 26.3; HRMS (ESI): m/z calcd. for $\left[\mathrm{C}_{36} \mathrm{H}_{30} \mathrm{NNaO} \text { P }, \mathrm{M}+\mathrm{Na}\right]^{+}:$594.1805; found: 594.1810.

Optical Rotation: $[\alpha]^{25} \mathrm{D}=-38.0(\mathrm{c}=0.1$, THF). The absolute configuration of 9 was assigned by analogy to 3a. 94\% ee (HPLC condition: Chiralpak IA column, $n$-hexane $/ i-\operatorname{PrOH}=80: 20$, flow rate $=1 \mathrm{ml} / \mathrm{min}$, wavelength $=254 \mathrm{~nm}, \mathrm{t}_{\mathrm{R}}=13.9 \mathrm{~min}$ for major isomer, $t_{\mathrm{R}}=18.0 \mathrm{~min}$ for minor isomer). 


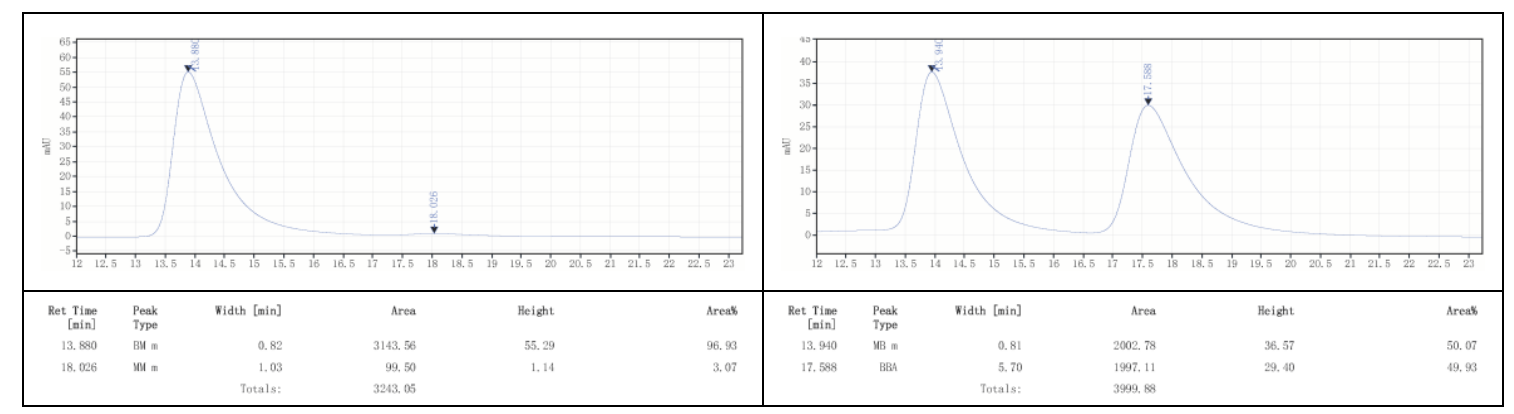

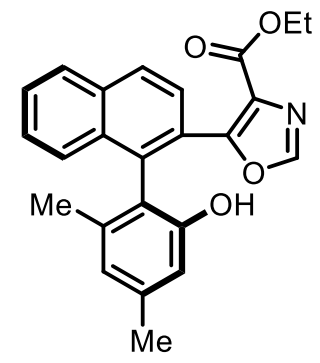

3a: $92 \%$ ee
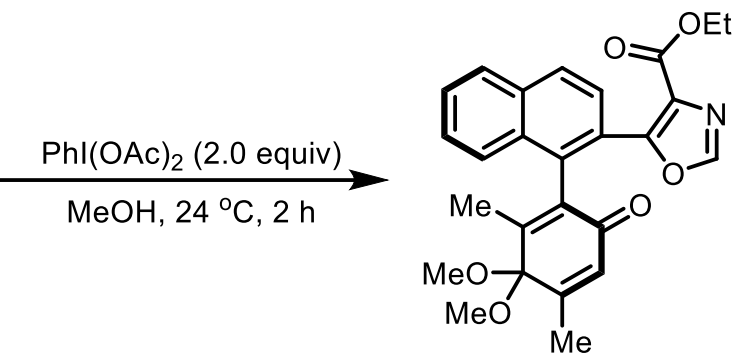

10: $83 \%, 92 \%$ ee

3a (38.7 mg, $0.10 \mathrm{mmol}, 92 \%$ ee) was added to the mixture of $\mathrm{PhI}(\mathrm{OAc})_{2}(66.4 \mathrm{mg}$, $0.20 \mathrm{mmol})$ in $\mathrm{MeOH}(2.0 \mathrm{~mL})$ at $0{ }^{\circ} \mathrm{C}$. The reaction mixture was stirred for $5 \mathrm{~min}$ at $0{ }^{\circ} \mathrm{C}$ and another $2 \mathrm{~h}$ at ambient temperature, then concentrated and purified by flash chromatography (PE/EtOAc 1:1) to afford $37.1 \mathrm{mg}$ of $\mathbf{1 0 .}$

\section{(S)-Ethyl}

5-(1-(3,3-dimethoxy-2,4-dimethyl-6-oxocyclohexa-1,4-dien-1-yl)naphthalen-2-yl)o xazole-4-carboxylate (10)<smiles>CCOC(=O)c1ncoc1-c1ccc2ccccc2c1C1=C(C)C(OC)(OC)C(OC)=CC1=O</smiles>

White solid, 83\% yield. MP: $183-185{ }^{\circ} \mathrm{C} ;{ }^{1} \mathbf{H}$ NMR (500 MHz, $\left.\mathrm{CDCl}_{3}\right): \delta 7.94(\mathrm{t}, J=$ $8.7 \mathrm{~Hz}, 2 \mathrm{H}), 7.77(\mathrm{~s}, 1 \mathrm{H}), 7.62(\mathrm{~d}, J=8.5 \mathrm{~Hz}, 1 \mathrm{H}), 7.58-7.52(\mathrm{~m}, 2 \mathrm{H}), 7.48-7.43(\mathrm{~m}$, $1 \mathrm{H}), 6.51(\mathrm{~d}, J=1.4 \mathrm{~Hz}, 1 \mathrm{H}), 4.30(\mathrm{q}, J=7.1 \mathrm{~Hz}, 2 \mathrm{H}), 3.25(\mathrm{~s}, 3 \mathrm{H}), 2.61(\mathrm{~s}, 3 \mathrm{H}), 1.98$ $(\mathrm{d}, J=1.3 \mathrm{~Hz}, 3 \mathrm{H}), 1.46(\mathrm{~s}, 3 \mathrm{H}), 1.23(\mathrm{t}, J=7.1 \mathrm{~Hz}, 3 \mathrm{H}) ;{ }^{13} \mathbf{C} \mathbf{N M R}(126 \mathrm{MHz}$, $\left.\mathrm{CDCl}_{3}\right): \delta 183.3,161.1,156.2,154.8,153.9,149.9,140.3,134.3,134.1,131.9,131.4$, 
128.7, 128.6, 128.2, 128.0, 127.6, 127.3, 125.3, 124.4, 98.2, 61.4, 51.2, 50.2, 16.5, 14.1, 13.7; HRMS (ESI): m/z calcd. for $\left[\mathrm{C}_{26} \mathrm{H}_{25} \mathrm{NNaO}_{6}, \mathrm{M}+\mathrm{Na}\right]^{+}: 470.1574$; found: 470.1575 .

Optical Rotation: $[\alpha]^{25}=+62.7(\mathrm{c}=0.3$, THF). The absolute configuration of $\mathbf{1 0}$ was assigned by analogy to 3a. 92\% ee (HPLC condition: Chiralpak IA column, $n$-hexane $/ i-\operatorname{PrOH}=80: 20$, flow rate $=1 \mathrm{ml} / \mathrm{min}$, wavelength $=254 \mathrm{~nm}, \mathrm{t}_{\mathrm{R}}=9.2 \mathrm{~min}$ for minor isomer, $t_{\mathrm{R}}=12.1 \mathrm{~min}$ for major isomer).

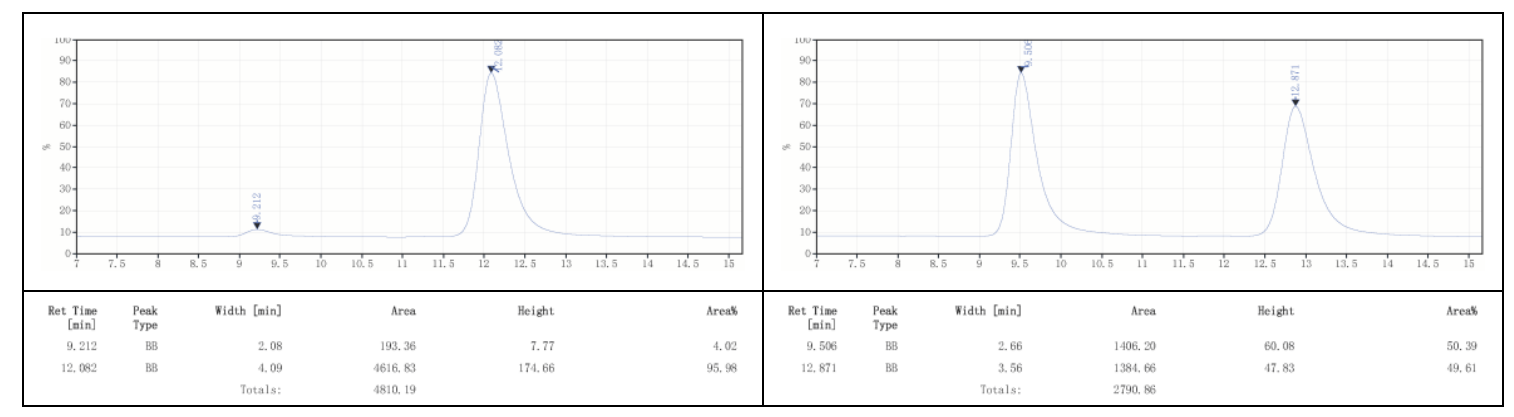

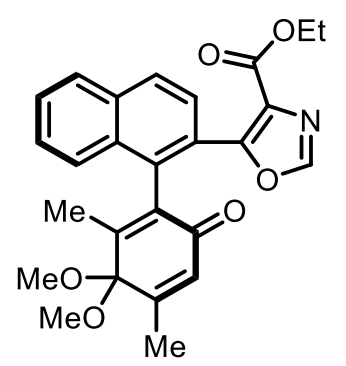

10: $92 \%$ ee
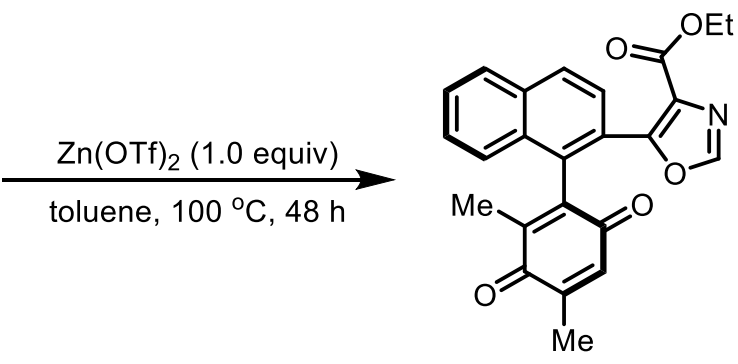

11: $91 \%, 89 \%$ ee

$\mathrm{Zn}(\mathrm{OTf})_{2}(18.2 \mathrm{mg}, 0.05 \mathrm{mmol})$ was added to the mixture of $\mathbf{1 0}(22.4 \mathrm{mg}, 0.05 \mathrm{mmol}$, $92 \%$ ee) in anhydrous toluene $(1.0 \mathrm{~mL})$ at $24{ }^{\circ} \mathrm{C}$. The reaction mixture was stirred at $100{ }^{\circ} \mathrm{C}$ for $48 \mathrm{~h}$, then concentrated and purified by flash chromatography (PE/EtOAc 1:1) to afford $18.2 \mathrm{mg}$ of $\mathbf{1 1 .}$

\section{(S)-Ethyl}

5-(1-(2,4-dimethyl-3,6-dioxocyclohexa-1,4-dien-1-yl)naphthalen-2-yl)oxazole-4-ca rboxylate (11) 


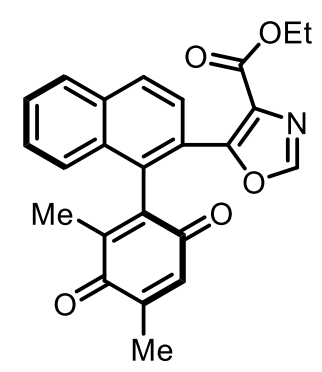

11

Yellow oil, $91 \%$ yield. ${ }^{1} \mathbf{H}$ NMR $\left(500 \mathrm{MHz}, \mathrm{CDCl}_{3}\right): \delta 7.99$ (d, $\left.J=8.5 \mathrm{~Hz}, 1 \mathrm{H}\right), 7.95$ $(\mathrm{d}, J=8.2 \mathrm{~Hz}, 1 \mathrm{H}), 7.81(\mathrm{~s}, 1 \mathrm{H}), 7.70(\mathrm{~d}, J=8.5 \mathrm{~Hz}, 1 \mathrm{H}), 7.61-7.56(\mathrm{~m}, 1 \mathrm{H})$, 7.55-7.46 (m, 2H), $6.68(\mathrm{q}, J=1.4 \mathrm{~Hz}, 1 \mathrm{H}), 4.29(\mathrm{q}, J=7.1 \mathrm{~Hz}, 2 \mathrm{H}), 2.12(\mathrm{~d}, J=1.5$ $\mathrm{Hz}, 3 \mathrm{H}), 1.65$ (s, 3H), 1.26 (t, $J=7.1 \mathrm{~Hz}, 3 \mathrm{H}) ;{ }^{13} \mathbf{C} \mathbf{N M R}\left(126 \mathrm{MHz}, \mathrm{CDCl}_{3}\right): \delta$ 187.6, $185.9,161.2,155.4,150.1,146.1,144.3,142.0,134.2,133.5,132.2,131.0,128.80$, $128.77,128.71,127.8,127.7,127.5,125.5,124.3,61.5,16.2,14.2,13.8$; HRMS (ESI): $\mathrm{m} / \mathrm{z}$ calcd. for $\left[\mathrm{C}_{24} \mathrm{H}_{19} \mathrm{NNaO}_{5}, \mathrm{M}+\mathrm{Na}\right]^{+}: 424.1155$; found: 424.1154 .

Optical Rotation: $[\alpha]^{25}=+92.2(\mathrm{c}=0.3$, THF). The absolute configuration of 11 was assigned by analogy to 3a. $89 \%$ ee (HPLC condition: Chiralpak IB N-5 column, $n$-hexane $/ i-\operatorname{PrOH}=80: 20$, flow rate $=1 \mathrm{ml} / \mathrm{min}$, wavelength $=254 \mathrm{~nm}, \mathrm{t}_{\mathrm{R}}=22.3 \mathrm{~min}$ for minor isomer, $t_{R}=31.3 \mathrm{~min}$ for major isomer).

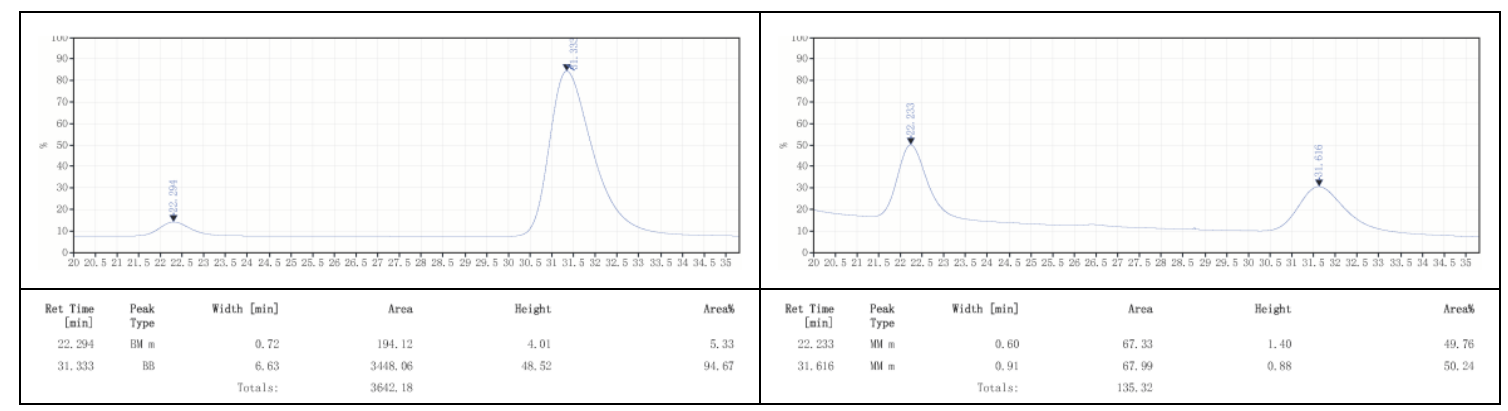

\section{X-ray crystallographic analysis and determination of configuration of $3 a$}

The absolute configuration of $\mathbf{3 a}(\boldsymbol{S})$ was assigned by X-ray crystallographic analysis of a single crystal of $\mathbf{3 a}$ (Figure S1). The crystal was prepared from the solution of $\mathbf{3 a}$ in hexanes at ambient temperature. 


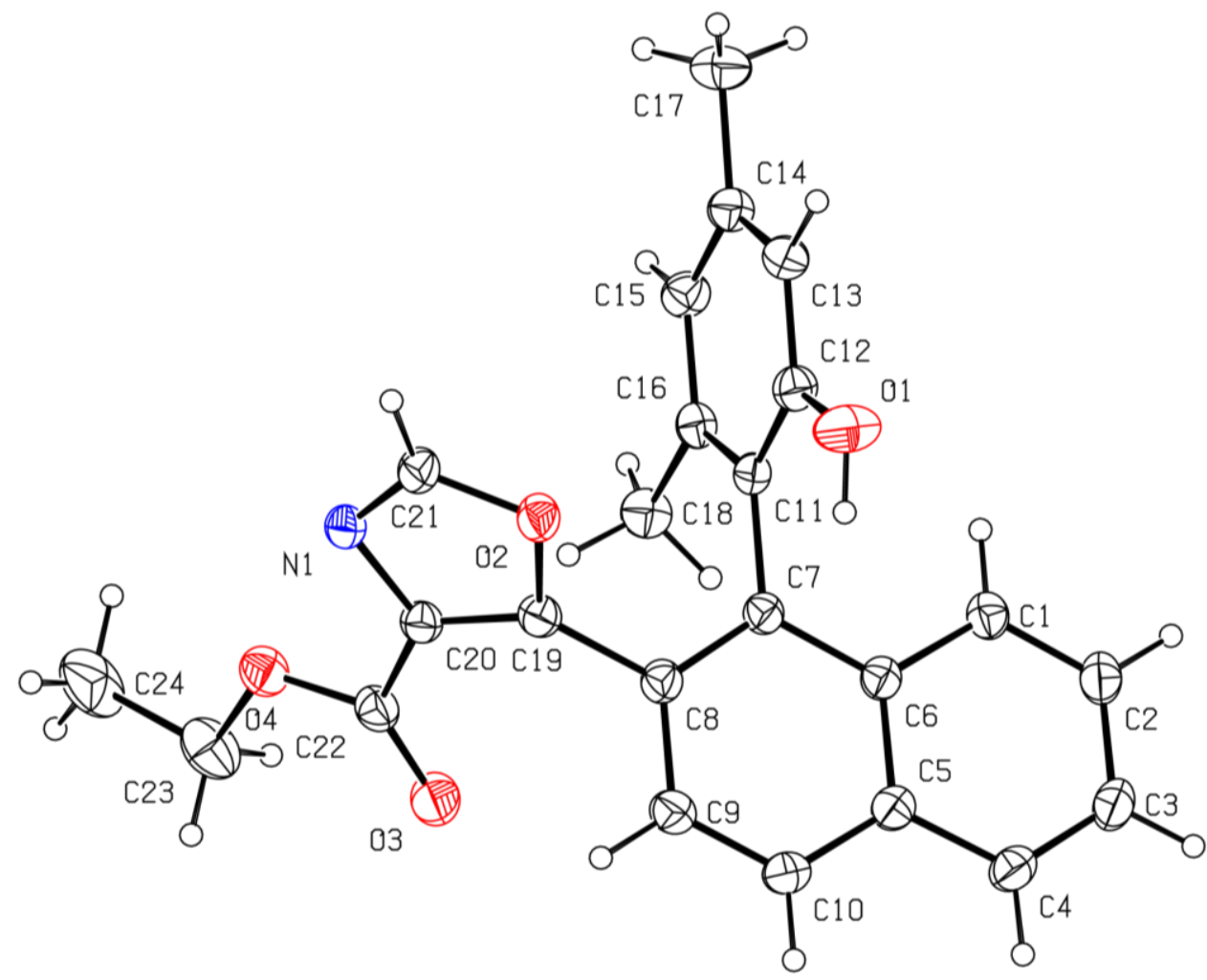

Figure S1. X-ray structure of 3a (ellipsoid contour at 30\% probability)

Table S1. Crystal data and structure refinement for 200811_Liao_DKR_0m

\begin{tabular}{ll}
\hline Identification code & 200811_Liao_DKR_Om \\
Empirical formula & $\mathrm{C}_{24} \mathrm{H}_{21} \mathrm{NO}_{4}$ \\
Formula weight & 387.42 \\
Temperature/K & 170.0 \\
Crystal system & orthorhombic \\
Space group & $\mathrm{P} 22_{1} 2_{21}$ \\
$\mathrm{a} / \AA$ & $12.4878(6)$ \\
$\mathrm{b} / \AA$ & $14.9550(7)$ \\
$\mathrm{c} / \AA$ & $21.5298(10)$ \\
$\alpha /{ }^{\circ}$ & 90 \\
$\beta /{ }^{\circ}$ & 90 \\
$\gamma /{ }^{\circ}$ & 90 \\
Volume $/ \AA^{3}$ & $4020.8(3)$ \\
$\mathrm{Z}$ & 8
\end{tabular}




$\begin{array}{ll}\rho_{\text {calcg } / \mathrm{cm}^{3}} & 1.280 \\ \mu / \mathrm{mm}^{-1} & 0.708 \\ \mathrm{~F}(000) & 1632.0 \\ \text { Crystal size } / \mathrm{mm}^{3} & 0.19 \times 0.14 \times 0.12 \\ \text { Radiation } & \mathrm{CuK} \alpha(\lambda=1.54178) \\ 2 \theta \text { range for data collection } /{ }^{\circ} & 7.196 \text { to } 136.872 \\ \text { Index ranges } & -15 \leq \mathrm{h} \leq 15,-17 \leq \mathrm{k} \leq 18,-25 \leq 1 \leq 25 \\ \text { Reflections collected } & 39930 \\ \text { Independent reflections } & 7347\left[\mathrm{R}_{\text {int }}=0.0343, \mathrm{R}_{\text {sigma }}=0.0227\right] \\ \text { Data/restraints/parameters } & 7347 / 1 / 533 \\ \text { Goodness-of-fit on } \mathrm{F}^{2} & 1.031 \\ \text { Final R indexes [I }>=2 \sigma(\mathrm{I})] & \mathrm{R}_{1}=0.0323, \mathrm{wR}_{2}=0.0871 \\ \text { Final R indexes [all data] } & \mathrm{R}_{1}=0.0332, \mathrm{wR}_{2}=0.0881 \\ \text { Largest diff. peak/hole } / \mathrm{e} \AA^{-3} & 0.14 /-0.15 \\ \text { Flack parameter } & -0.07(5)\end{array}$

\section{References}

1. Housseman, C.; Zhu, J. Synlett 2006, 1777-1779.

2. (a) Yu, C.; Huang, H.; Li, X.; Zhang, Y.; Wang, W. J. Am. Chem. Soc. 2016, 138, 6956-6959. (b) Chen, G.-Q.; Lin, B.-J.; Huang, J.-M.; Zhao, L.-Y.; Chen, Q.-S.; Jia, S.-P.; Yin, Q.; Zhang, X. J. Am. Chem. Soc. 2018, 140, 8064-8068.

3. (a) Shao, P.-L.; Liao, J.-Y.; Ho, Y. A.; Zhao, Y. Angew. Chem. Int. Ed. 2014, 53, 5435-5439. (b) Sladojevich, F.; Trabocchi, A.; Guarna, A.; Dixon, D. J. J. Am. Chem. Soc. 2011, 133, 1710-1713.

4. (a) Becke, A. D. J. Chem. Phys. 1993, 98, 5648-5652. (b) Lee, C.; Yang, W.; Parr, R. G. Phys. Rev. B 1988, 37, 785-789.

5. (a) Zhao, Y.; Truhlar, D. G. Theor. Chem. Acc. 2008, 120, 215-241. (b) Zhao, Y.; Truhlar, D. G. Acc. Chem. Res. 2008, 41, 157-167.

6. (a) Peverati, R.; Truhlar, D. G. Phys. Chem. Chem. Phys. 2012, 14, 11363-11370. 
(b) Lin, Y.-S.; Tsai, C.-W.; Li, G.-D.; Chai, J.-D. J. Chem. Phys. 2012, 136, 154109. (c) Steckel, J. A. J. Phys. Chem. A 2012, 116, 11643-11650. (d) Zhao, Y.; Ng, H. T.; Peverati, R.; Truhlar, D. G. J. Chem. Theory Comput. 2012, 8, 2824-2834. (e) Yu, Z.; Lan, Y. J. Org. Chem. 2013, 78, 11501-11507. (f) Liu, S.; Lei, Y.; Qi, X.; Lan, Y. J. Phys. Chem. A 2014, 118, 2638-2645. (g) Xi, Y.; Su, Y.; Yu, Z.; Dong, B.; McClain, E. J.; Lan, Y.; Shi, X. Angew. Chem. Int. Ed. 2014, 53, 9817-9821. (h) Long, R.; Huang, J.; Shao, W.; Liu, S.; Lan, Y.; Gong, J.; Yang, Z. Nat. Commun. 2014, 5, 5707. (i) Qi, X.; Zhang, H.; Shao, A.; Zhu, L.; Xu, T.; Gao, M.; Liu, C.; Lan, Y. ACS Catal. 2015, 5, 6640-6647. (j) Fu, J.; Gu, Y.; Yuan, H.; Luo, T.; Liu, S.; Lan, Y.; Gong, J.; Yang, Z. Nat. Commun. 2015, 6, 8617.

7. (a) Cancès, E.; Mennucci, B.; Tomasi, J. J. Chem. Phys. 1997, 107, 3032-3041. (b) Cossi, M.; Barone, V.; Cammi, R.; Tomasi, J. Chem. Phys. Lett. 1996, 255, 327-335. (c) Barone, V.; Cossi, M.; Tomasi, J. J. Comput. Chem. 1998, 19, 404-417.

8. For NMR data of the anti diastereomer, see: (a) Yamada, T.; Nagata, T.; Sugi, K. D.; Yorozu, K.; Ikeno, T.; Ohtsuka, Y.; Miyazaki, D.; Mukaiyama, T. Chem. Eur. J. 2003, 9, 4485-4509. For NMR data of the syn diastereomer, see: (b) Sevrain, N.; Volle, J.-N.; Pirat, J.-L.; Ayad, T.; Virieux, D. Eur. J. Org. Chem. 2018, $2267-2272$. 
IX. NMR spectra of the products

${ }^{1} \mathbf{H}$ NMR $\left(500 \mathrm{MHz}, \mathrm{CDCl}_{3}\right)$

㤎思息

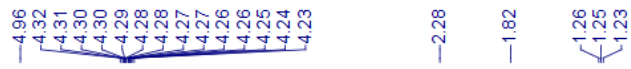<smiles>CCOC(=O)c1ncoc1-c1c(O)cc([N+](=O)[O-])cc1[N+](=O)[O-]</smiles>

$3 a$

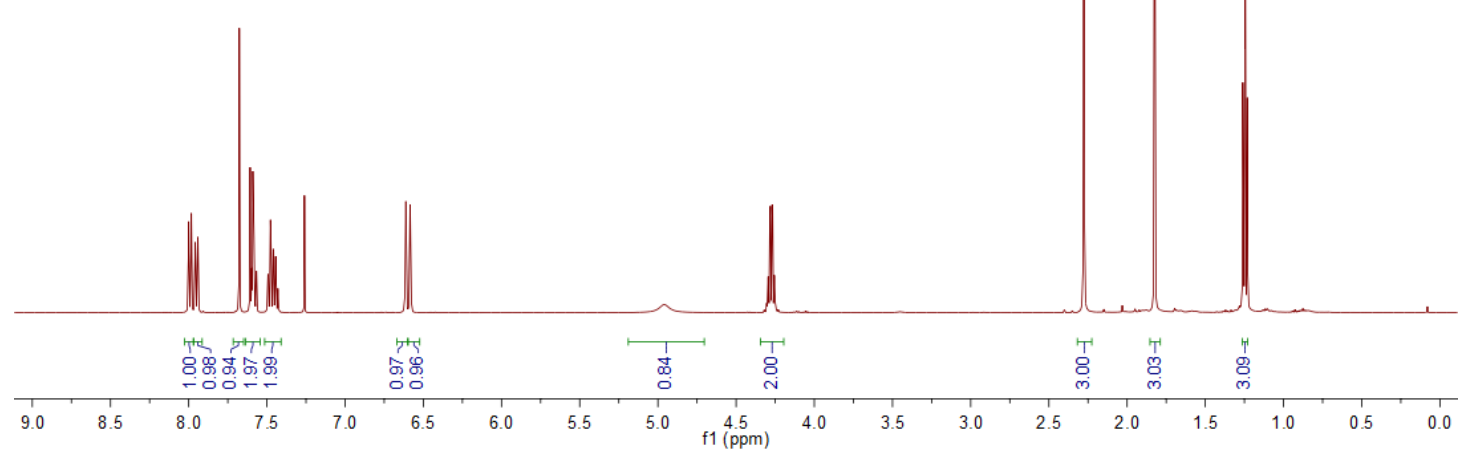

${ }^{13} \mathbf{C}$ NMR (126 MHz, $\left.\mathrm{CDCl}_{3}\right)$

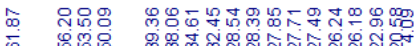

ए<smiles>CCOC(=O)c1ncoc1-c1c(O)cc([N+](=O)[O-])cc1[N+](=O)[O-]</smiles>

$3 a$

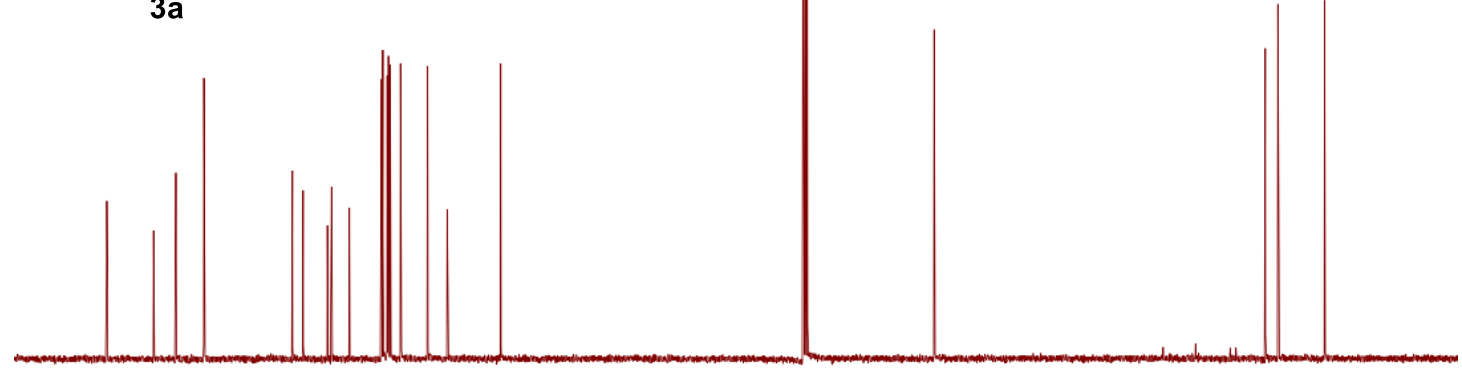

$\begin{array}{llllll}170 & 160 & 150 & 140 & 130 & 120\end{array}$

$110 \quad 100$

${ }_{\mathrm{f} 1(\mathrm{ppm})}^{80}$ 
${ }^{1} \mathbf{H}$ NMR $\left(500 \mathrm{MHz}, \mathrm{CDCl}_{3}\right)$

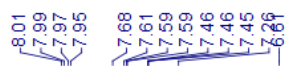

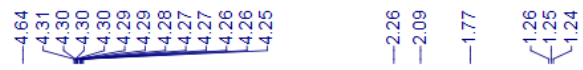

(O)

3b

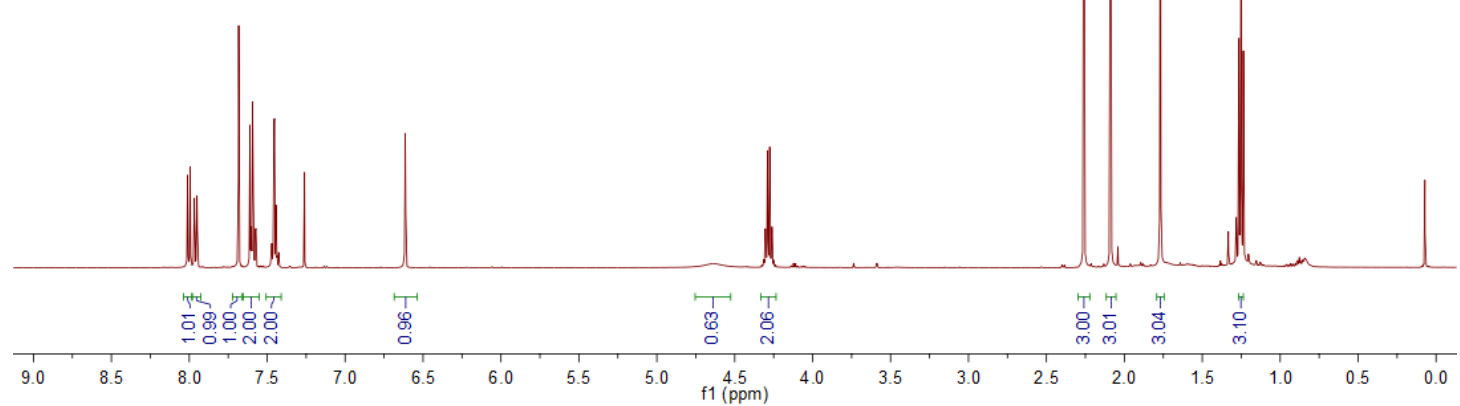

${ }^{13}$ C NMR (126 MHz, $\left.\mathrm{CDCl}_{3}\right)$

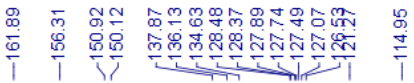

$\stackrel{\substack{6 \\ \hdashline}}{i}$

8요의

ํํㄴ둔

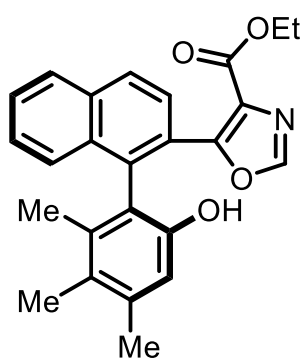

$3 b$
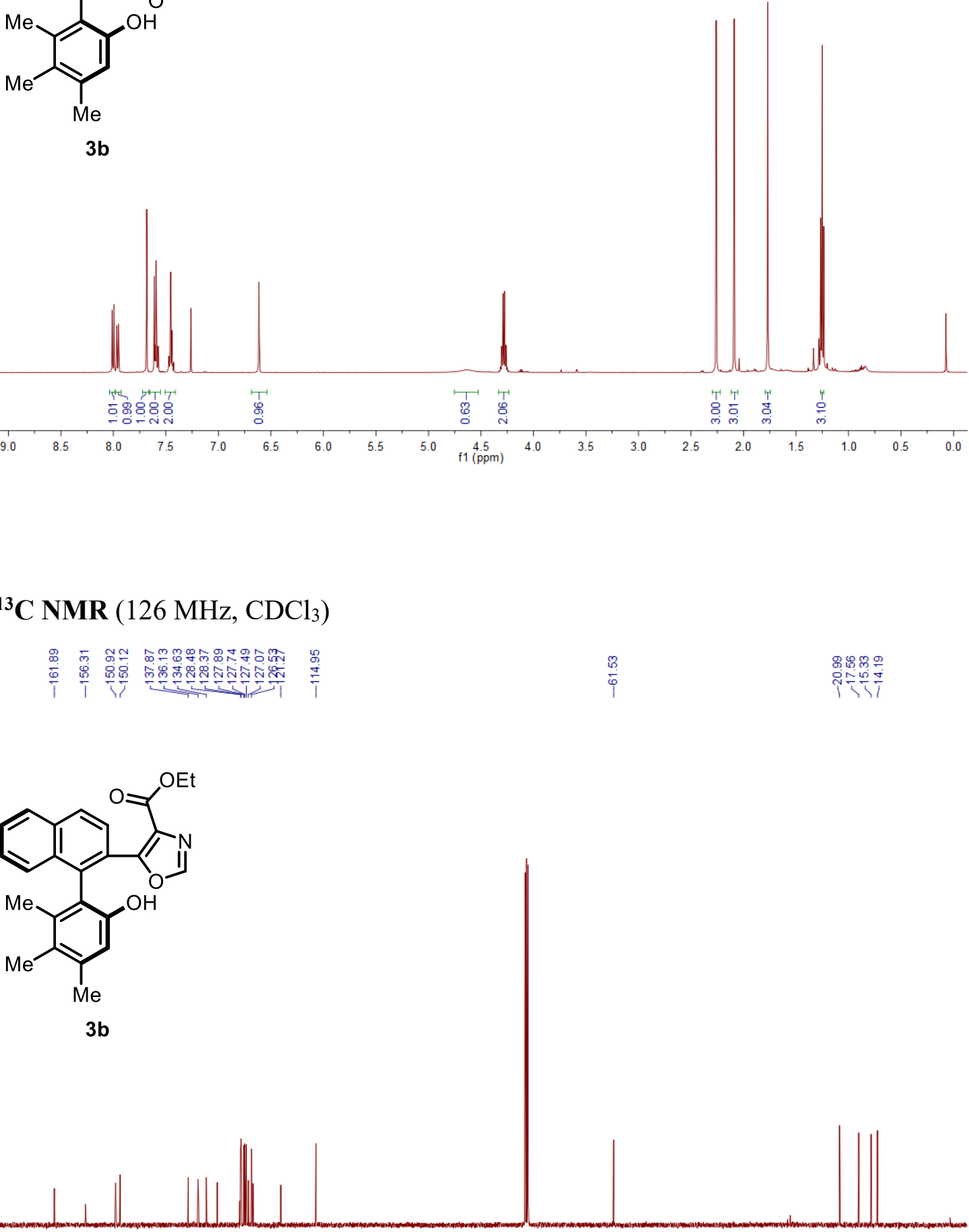

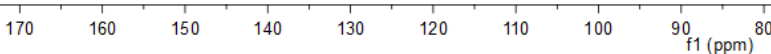


${ }^{1} \mathbf{H}$ NMR (400 MHz, $\left.\mathrm{CDCl}_{3}\right)$

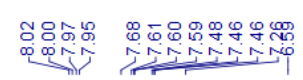

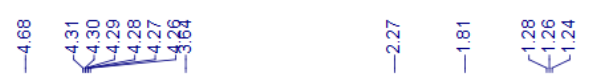<smiles>CCOC(=O)c1ncoc1-c1ccc2ccccc2c1-c1c(O)cc(C)c(OC)c1OC</smiles>

${ }^{13}$ C NMR (101 MHz, $\left.\mathrm{CDCl}_{3}\right)$

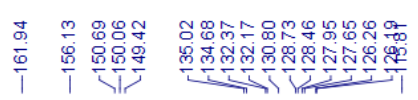
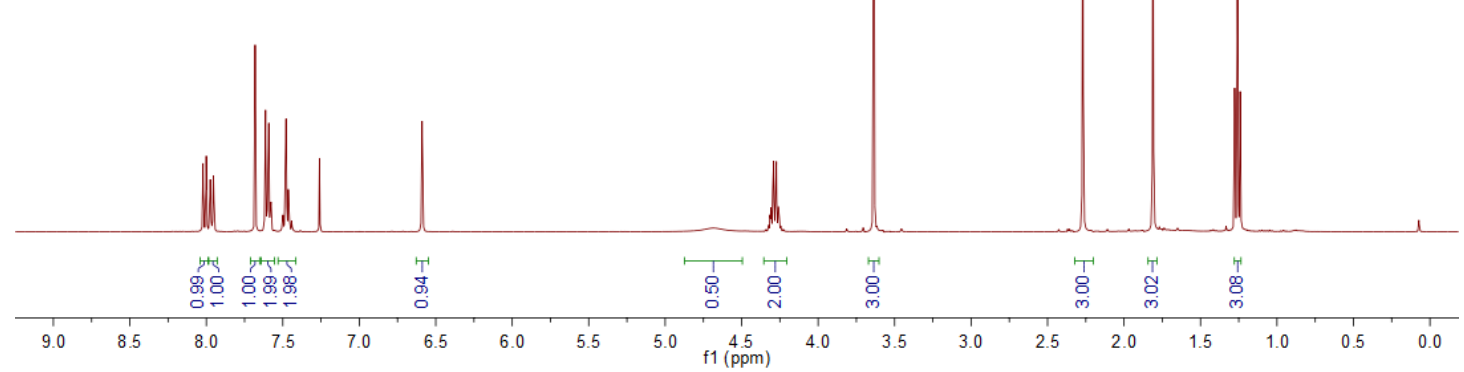<smiles>CCOC(=O)c1ncoc1-c1ccc2ccccc2c1-c1c(O)cc([N+](=O)[O-])c(OC)c1OC</smiles>

$3 c$

品

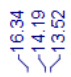

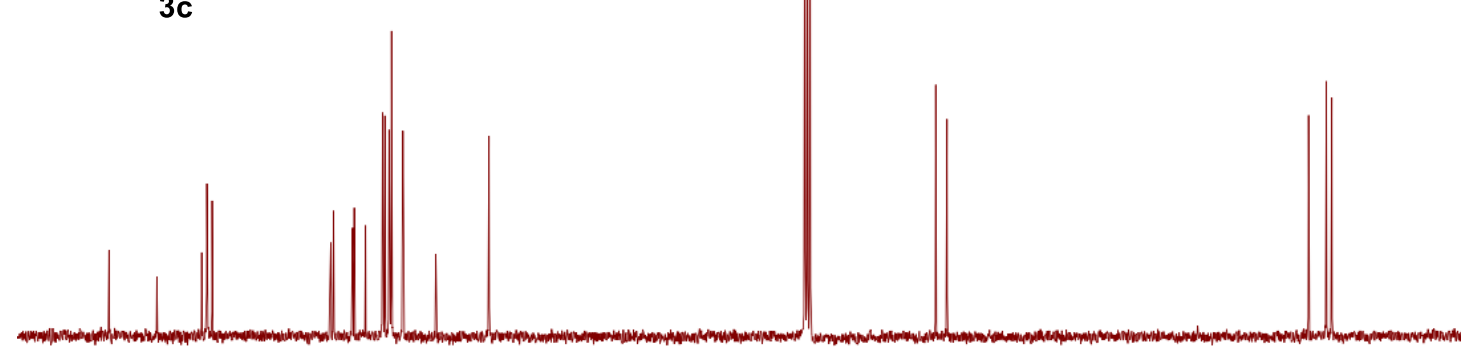

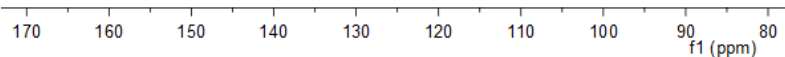


${ }^{1} \mathbf{H}$ NMR (500 MHz, $\left.\mathrm{CDCl}_{3}\right)$

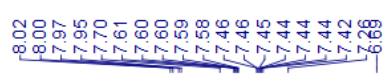

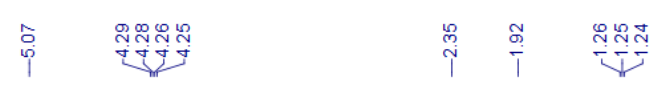

(1)

3d

${ }^{13} \mathbf{C ~ N M R}\left(126 \mathrm{MHz}, \mathrm{CDCl}_{3}\right)$

क.

ष<smiles>CCOC(=O)c1ncoc1-c1ccc2ccccc2c1-c1c(O)cc([N+](=O)[O-])c(Cl)c1C</smiles>

3d

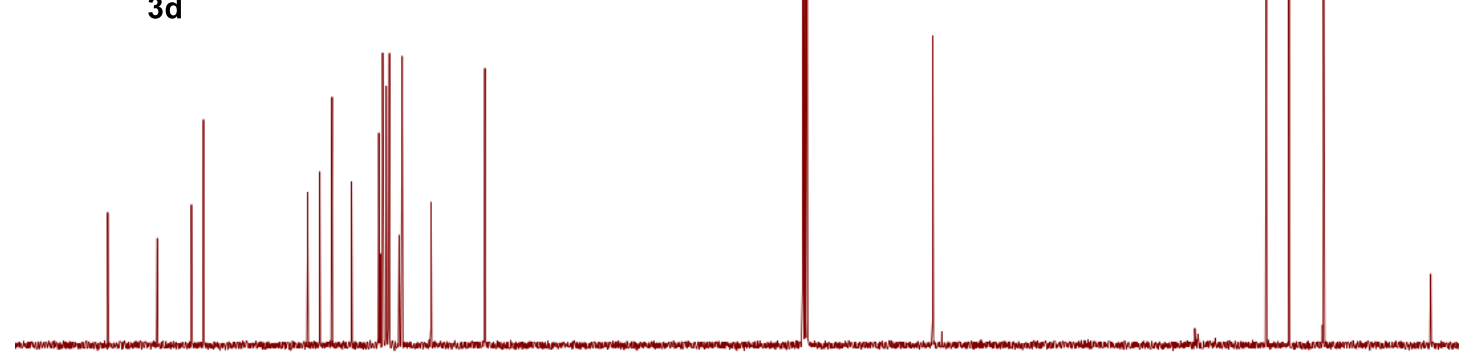

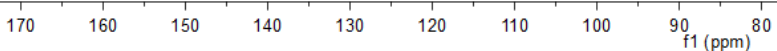

$\frac{\widetilde{6}}{i}$

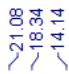

Et 
${ }^{1} \mathbf{H}$ NMR $\left(500 \mathrm{MHz}, \mathrm{CDCl}_{3}\right)$

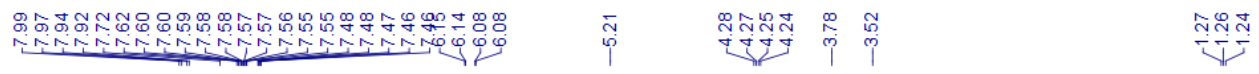<smiles>CCOC(=O)c1ncoc1-c1ccc2ccccc2c1-c1c(OC)cc(OC)cc1OC</smiles>

${ }^{13} \mathbf{C}$ NMR $\left(126 \mathrm{MHz}, \mathrm{CDCl}_{3}\right)$

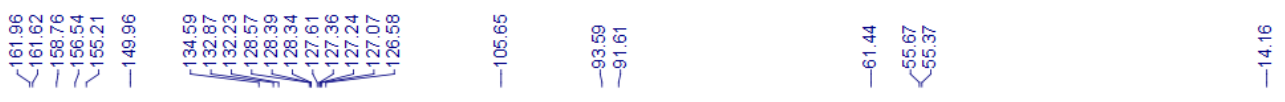<smiles>CCOC(=O)c1ncoc1-c1ccc2ccccc2c1-c1c(O)cc(OC)cc1OC</smiles>

$3 e$

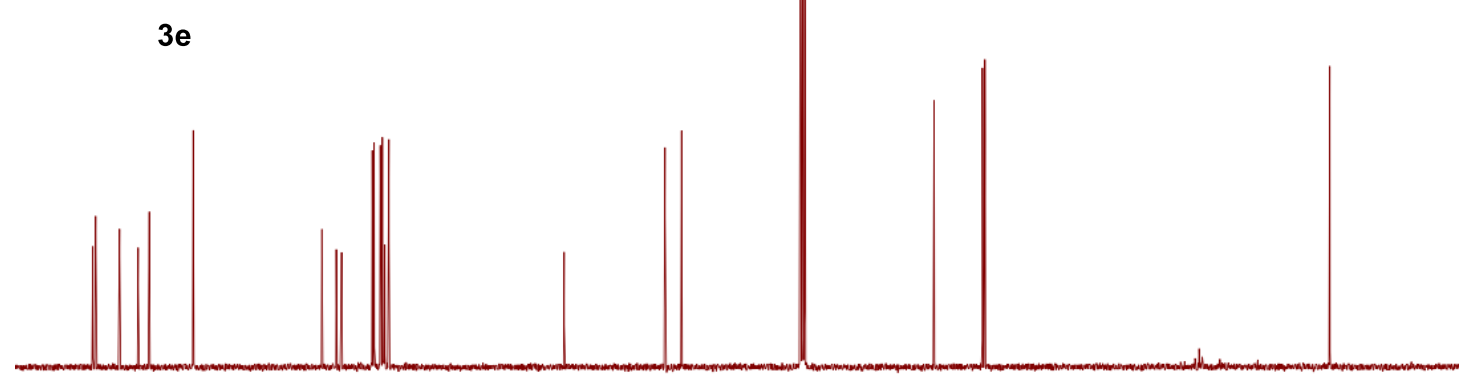

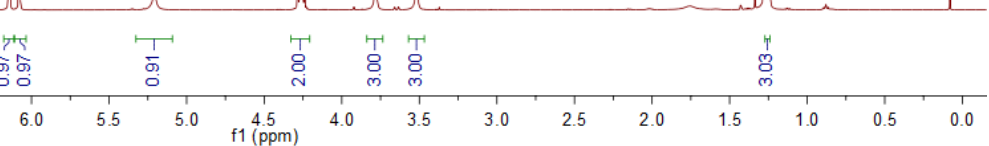

$\stackrel{T}{170}$
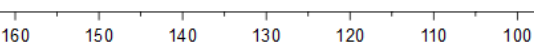

$90_{\mathrm{f} 1(\mathrm{ppm})} 80$ 
${ }^{1}$ H NMR (400 MHz, DMSO)

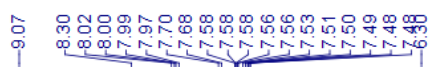

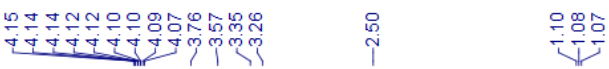<smiles>CCOC(=O)c1ncoc1-c1ccc2ccccc2c1-c1c(O)cc(OC)c(OC)c1OC</smiles>

${ }^{13}$ C NMR (126 MHz, DMSO)

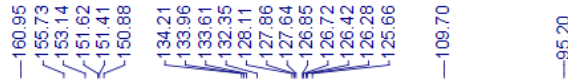

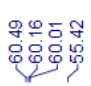

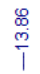<smiles>CCOC(=O)c1ncoc1-c1ccc2ccccc2c1-c1c(O)cc(OC)c(OC)c1OC</smiles>

$3 f$
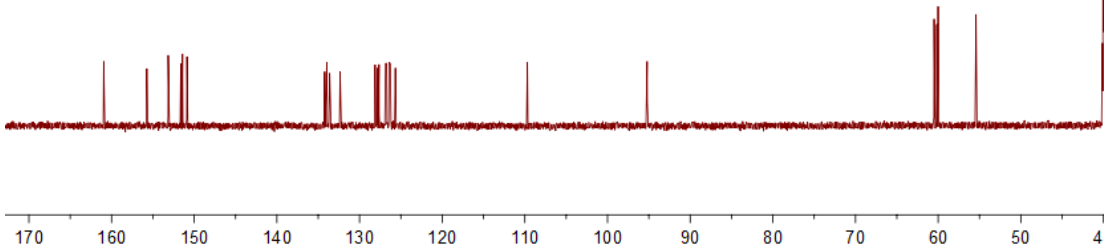

140
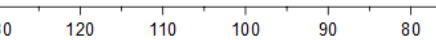
${ }^{1} \mathbf{H}$ NMR $\left(500 \mathrm{MHz}, \mathrm{CDCl}_{3}\right)$

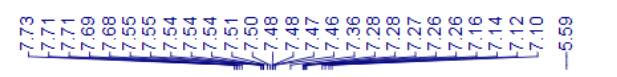

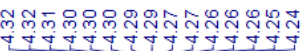

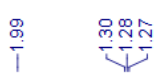<smiles>CCOC(=O)c1ncoc1-c1cccc(O)c1-c1c(O)ccc2ccccc12</smiles>

$3 g$

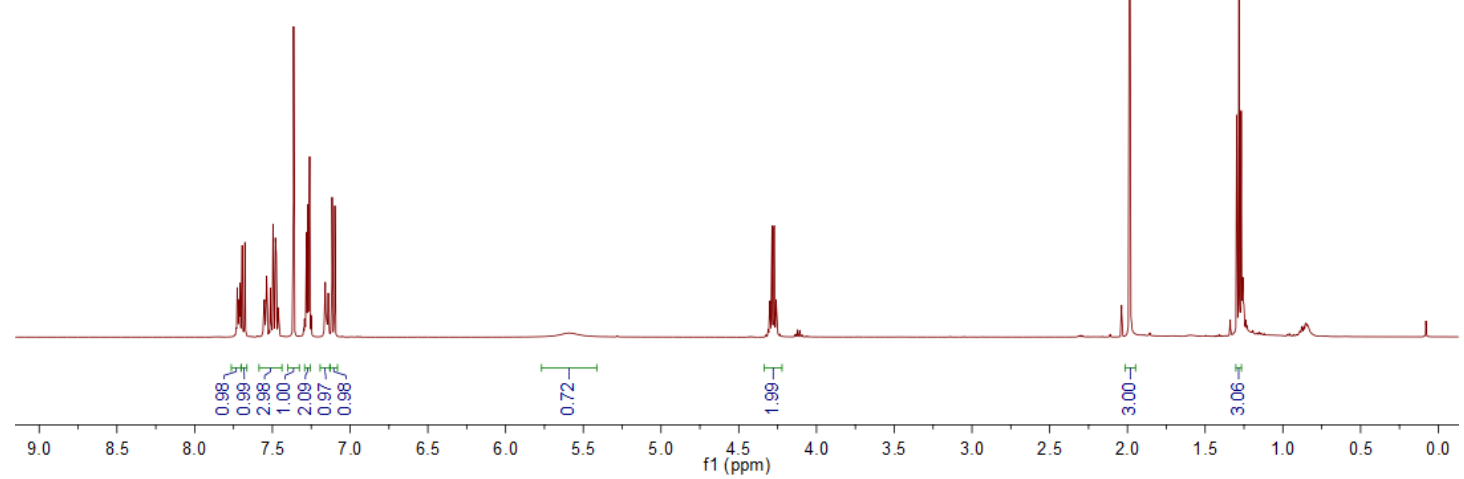

${ }^{13} \mathbf{C}$ NMR $\left(126 \mathrm{MHz}, \mathrm{CDCl}_{3}\right)$

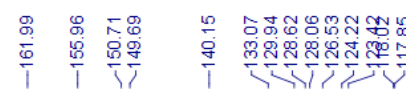

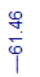

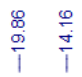<smiles>CCOC(=O)c1ncoc1-c1cccc(C)c1-c1c(O)ccc2ccccc12</smiles>

$3 g$

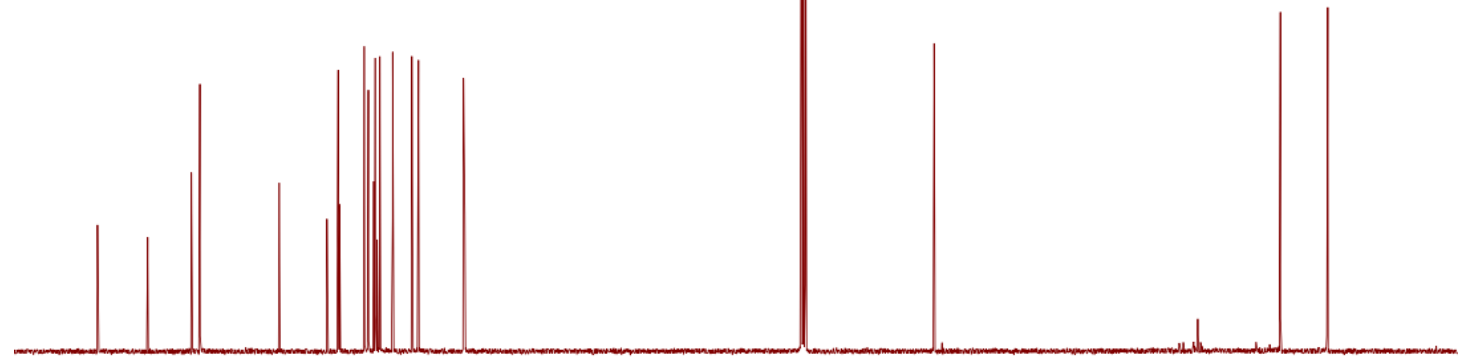

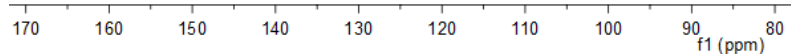


${ }^{1} \mathbf{H}$ NMR $\left(500 \mathrm{MHz}, \mathrm{CDCl}_{3}\right)$

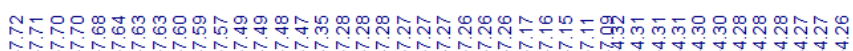

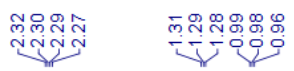<smiles>CCOC(=O)c1ncoc1-c1cccc(O)c1-c1c(CC)ccc2ccccc12</smiles>

$3 \mathrm{~h}$

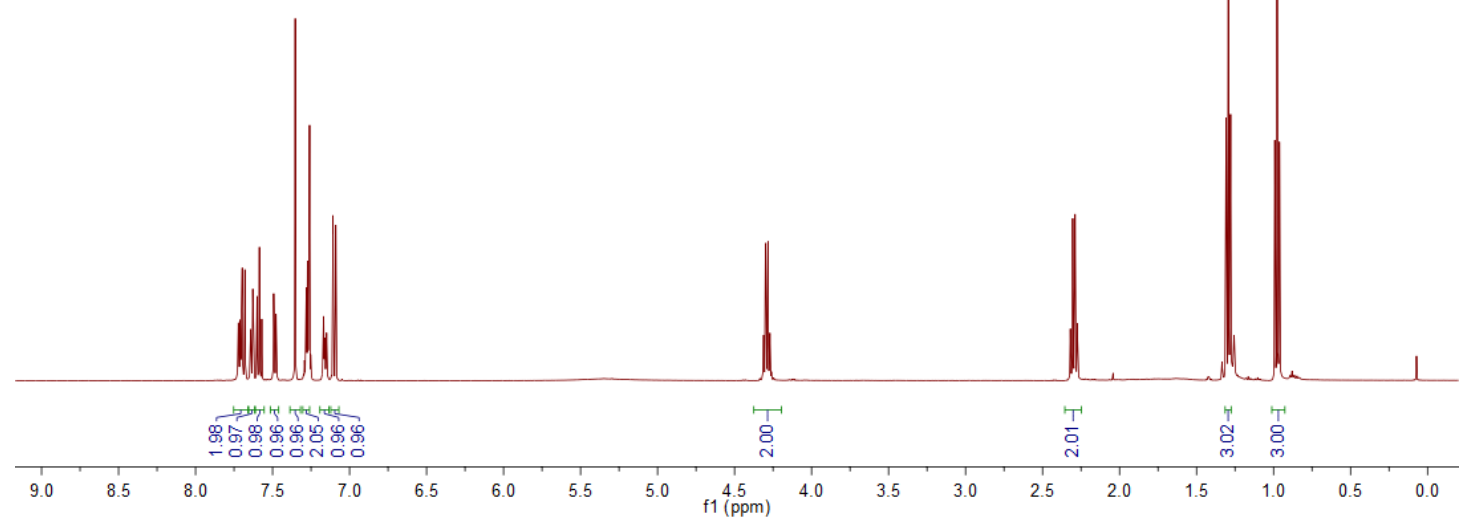

${ }^{13} \mathbf{C ~ N M R}\left(126 \mathrm{MHz}, \mathrm{CDCl}_{3}\right)$

ㄴ.

ᄂ

i<smiles>CCOC(=O)c1ncoc1-c1cccc(O)c1-c1c(CC)ccc2ccccc12</smiles>

$3 \mathrm{~h}$

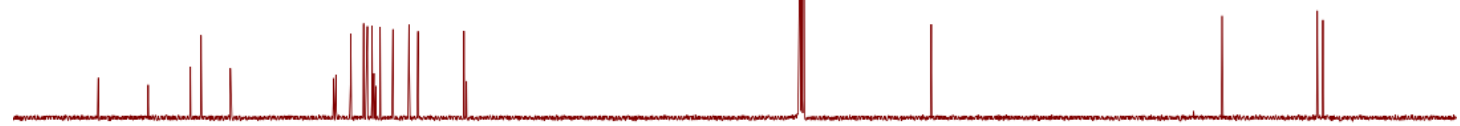

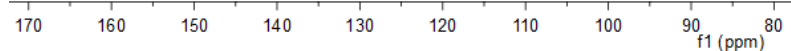


${ }^{1} \mathbf{H}$ NMR (500 MHz, $\left.\mathrm{CDCl}_{3}\right)$

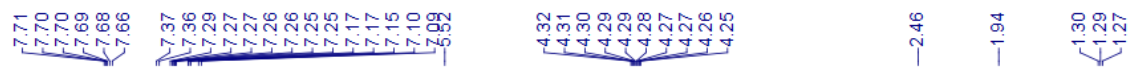<smiles>CCOC(=O)c1ncoc1-c1cc([N+](=O)[O-])cc([N+](=O)[O-])c1-c1c(O)ccc2ccccc12</smiles>

$3 \mathbf{i}$

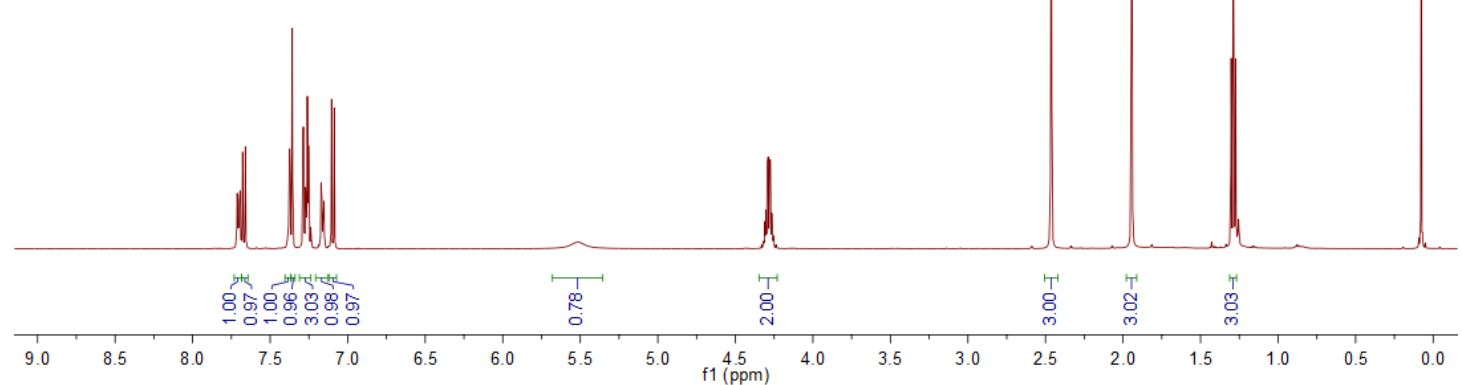

${ }^{13} \mathbf{C ~ N M R}\left(126 \mathrm{MHz}, \mathrm{CDCl}_{3}\right)$

กิ

品

$\frac{i}{\frac{1}{4}}$

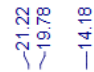<smiles>CCOC(=O)c1ncoc1-c1cc(C)cc([N+](=O)[O-])c1-c1c(O)ccc2ccccc12</smiles>

3i

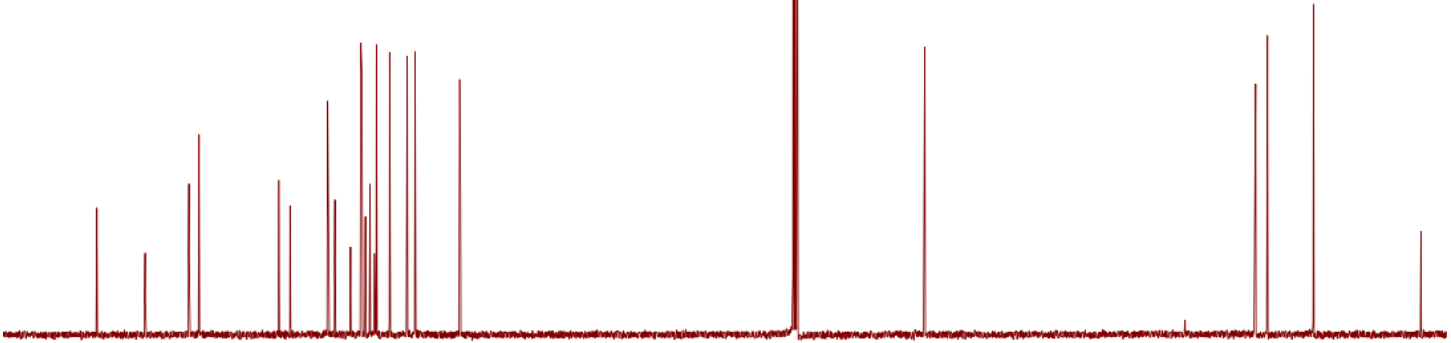

$\begin{array}{lllllllll}170 & 160 & 150 & 140 & 130 & 120 & 110 & 100 & 90 \\ \mathrm{f} 1(\mathrm{ppm}) & 80\end{array}$ 
${ }^{1} \mathbf{H}$ NMR (500 MHz, $\left.\mathrm{CDCl}_{3}\right)$

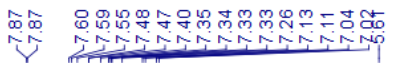<smiles>CCOC(=O)c1ncoc1-c1cccc(C)c1-c1c(O)ccc2cc(Br)ccc12</smiles>

3j

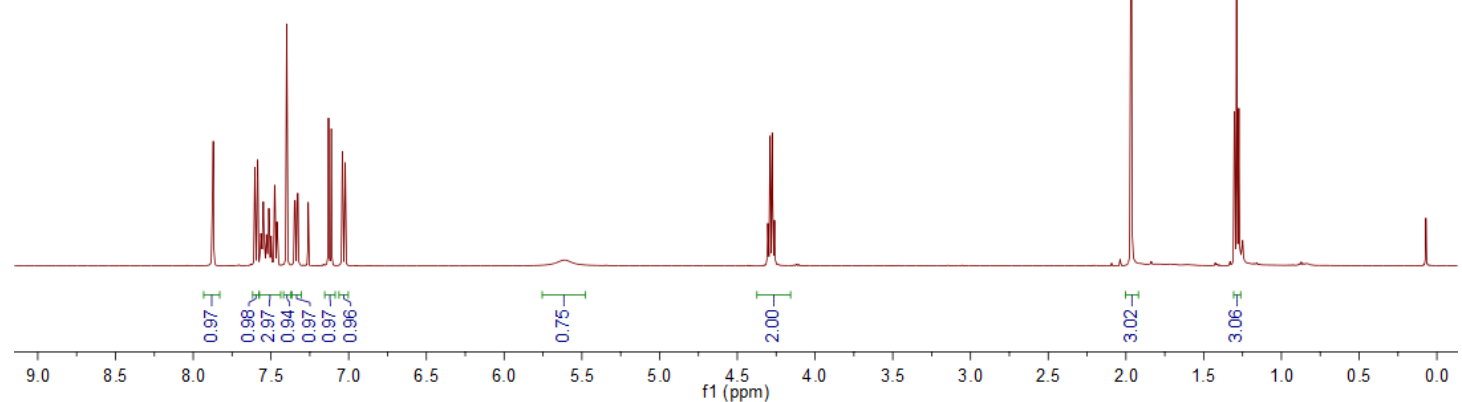

${ }^{13} \mathbf{C ~ N M R}\left(126 \mathrm{MHz}, \mathrm{CDCl}_{3}\right)$<smiles>CCOC(=O)c1ncoc1-c1cccc(C)c1-c1c(O)ccc2cc(Br)ccc12</smiles>

3j

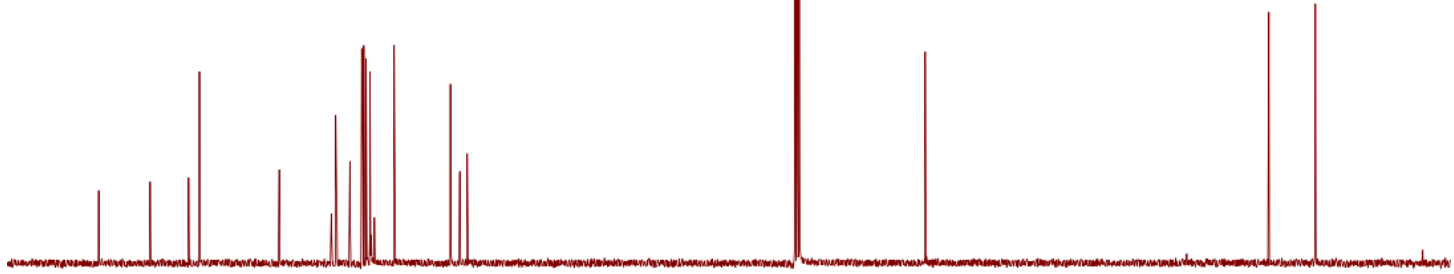

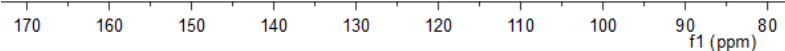


${ }^{1} \mathbf{H}$ NMR (500 MHz, $\left.\mathrm{CDCl}_{3}\right)$

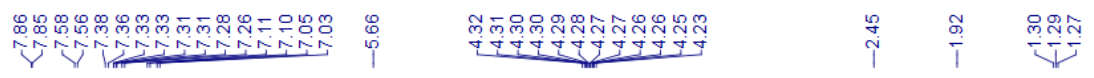<smiles>CCOC(=O)c1ncoc1-c1c(O)cc([N+](=O)[O-])cc1-c1c(O)ccc2cc(Br)ccc12</smiles>

3k

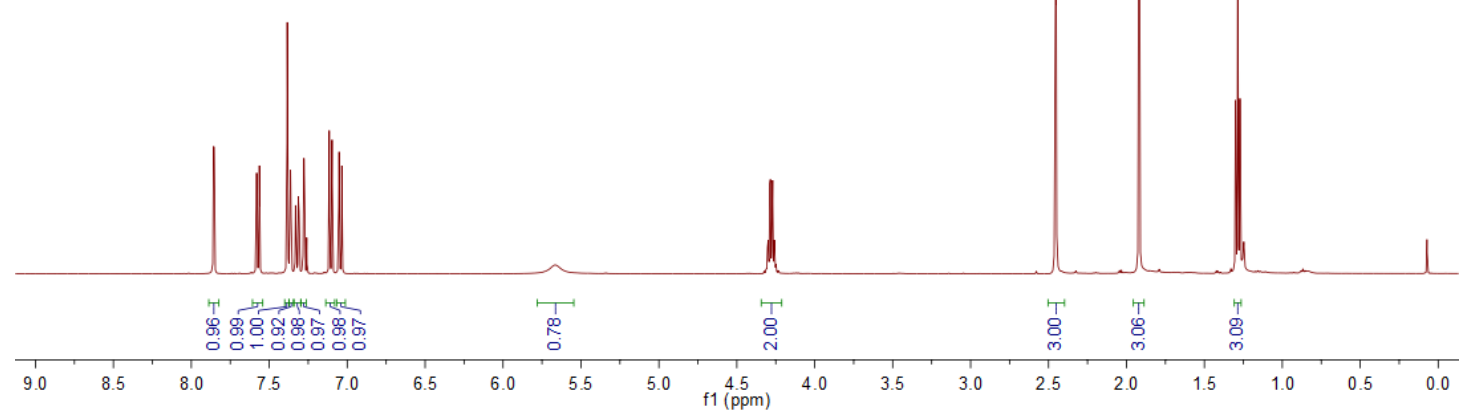

${ }^{13}$ C NMR (126 MHz, $\left.\mathrm{CDCl}_{3}\right)$

\%.

ए人

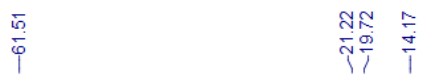<smiles>CCOC(=O)c1ncoc1-c1cc(C)cc(C)c1-c1c(O)ccc2cc(Br)ccc12</smiles>

$3 k$

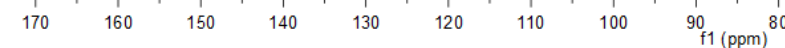


${ }^{1} \mathbf{H}$ NMR (500 MHz, $\left.\mathrm{CDCl}_{3}\right)$

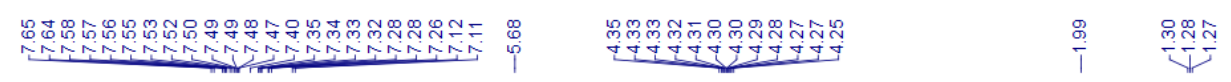<smiles>CCOC(=O)c1ncoc1-c1cccc(C)c1-c1c(O)ccc2ccc(Br)cc12</smiles>

3I

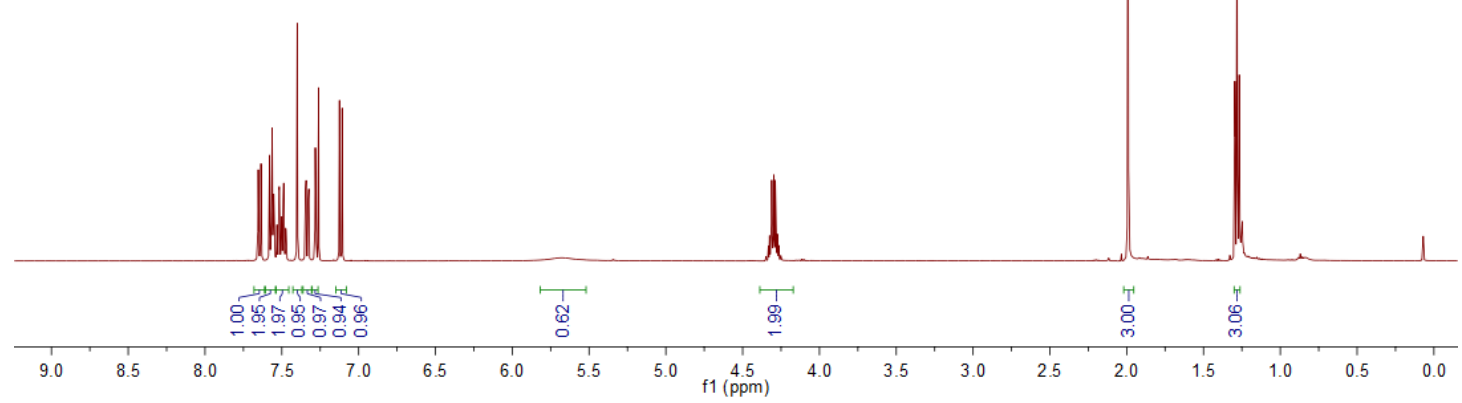

${ }^{13} \mathbf{C}$ NMR (126 MHz, $\left.\mathrm{CDCl}_{3}\right)$

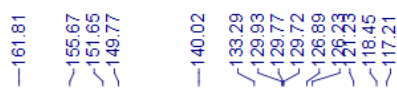

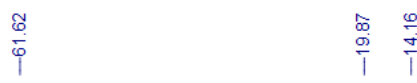

(1)

31

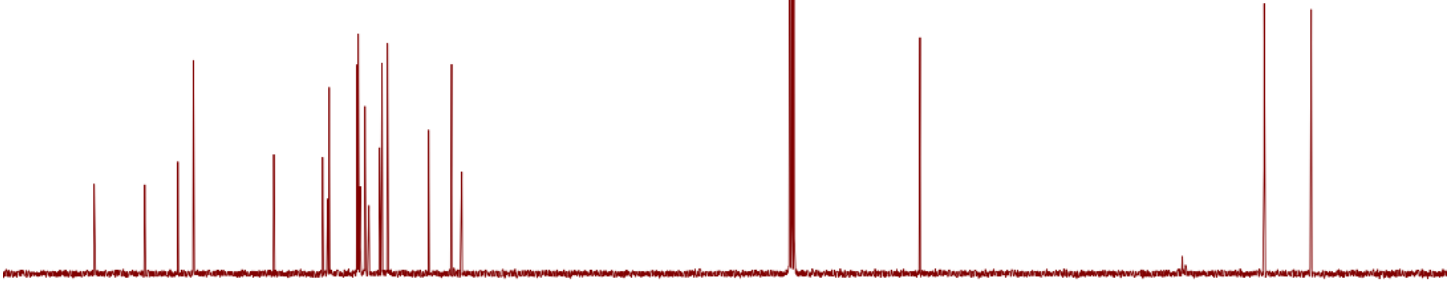

\begin{tabular}{lllllllll}
\hline 170 & 160 & 150 & 140 & 130 & 120 & 110 & 100 & 90 \\
$\mathrm{f} 1(\mathrm{ppm})$ & 10
\end{tabular} 
${ }^{1} \mathbf{H}$ NMR (500 MHz, $\mathrm{CDCl}_{3}$ )

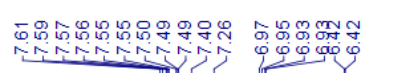

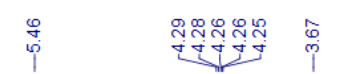

ฺิ<smiles>CCOC(=O)c1ncoc1-c1cccc(O)c1-c1c(O)ccc2ccc(OC)cc12</smiles>

$3 m$

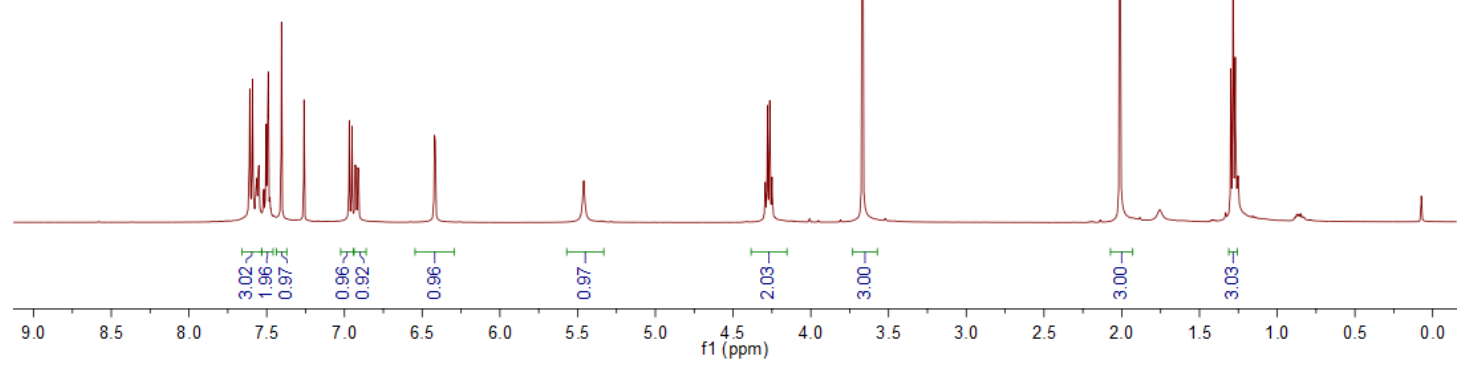

${ }^{13}$ C NMR (126 MHz, $\left.\mathrm{CDCl}_{3}\right)$

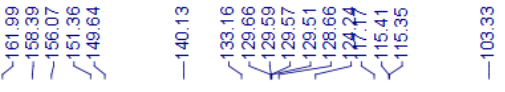
$\frac{19}{1} \frac{1}{9}$

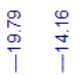<smiles>CCOC(=O)c1ncoc1-c1cccc(O)c1-c1c(O)ccc2ccc(OC)cc12</smiles>

$3 \mathrm{~m}$

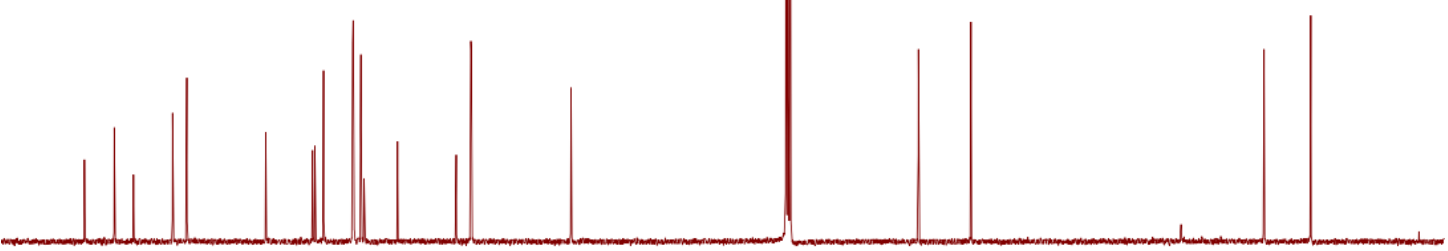

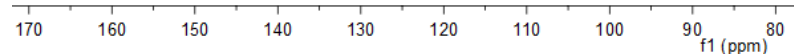


${ }^{1} \mathbf{H}$ NMR (500 MHz, $\left.\mathrm{CDCl}_{3}\right)$

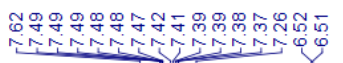

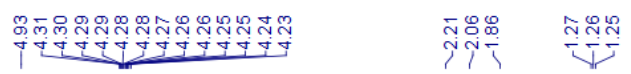

(1)

$3 n$

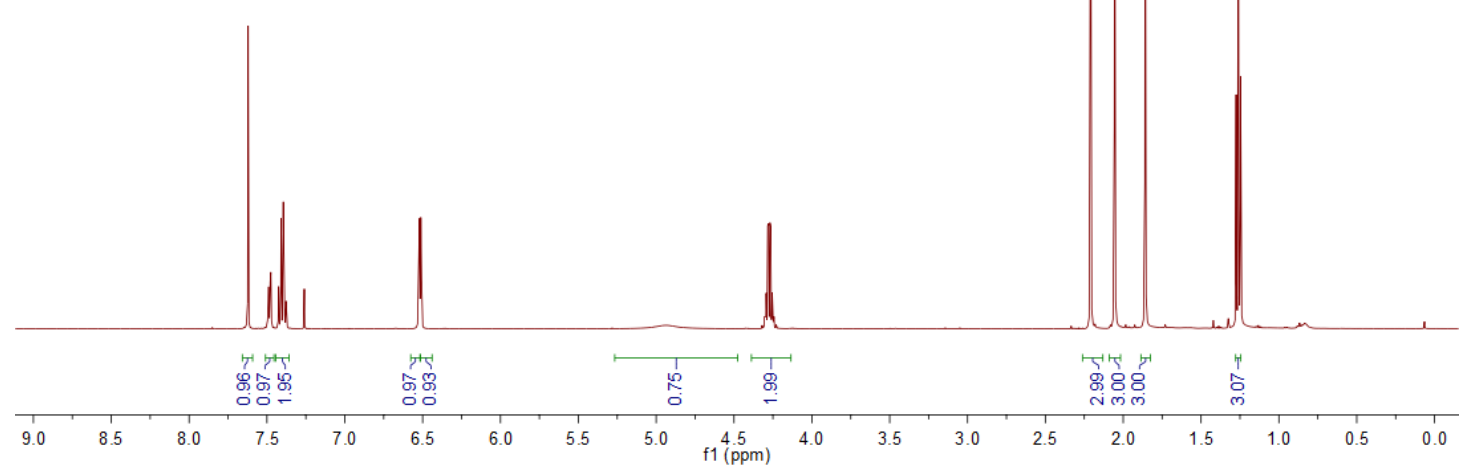

${ }^{13} \mathbf{C}$ NMR (126 MHz, $\left.\mathrm{CDCl}_{3}\right)$

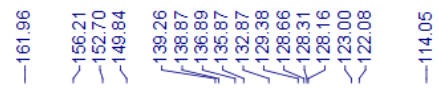

$\frac{\substack{9 \\ i}}{i}$

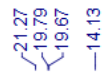<smiles>CCOC(=O)c1ncoc1-c1cccc(C)c1-c1c(C)cc(C)cc1O</smiles>

$3 n$

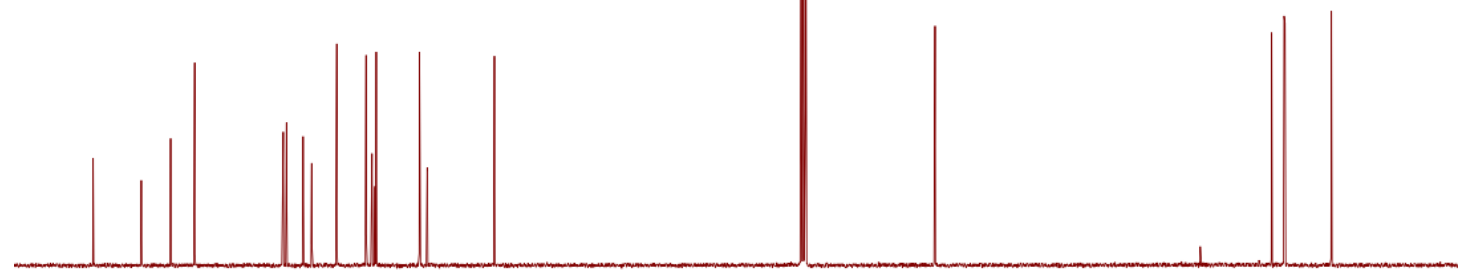

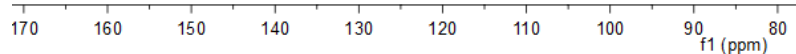


${ }^{1} \mathbf{H}$ NMR (500 MHz, $\left.\mathrm{CDCl}_{3}\right)$

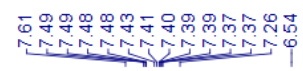

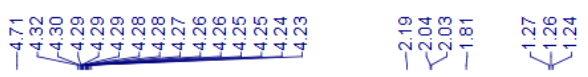

(1)

30

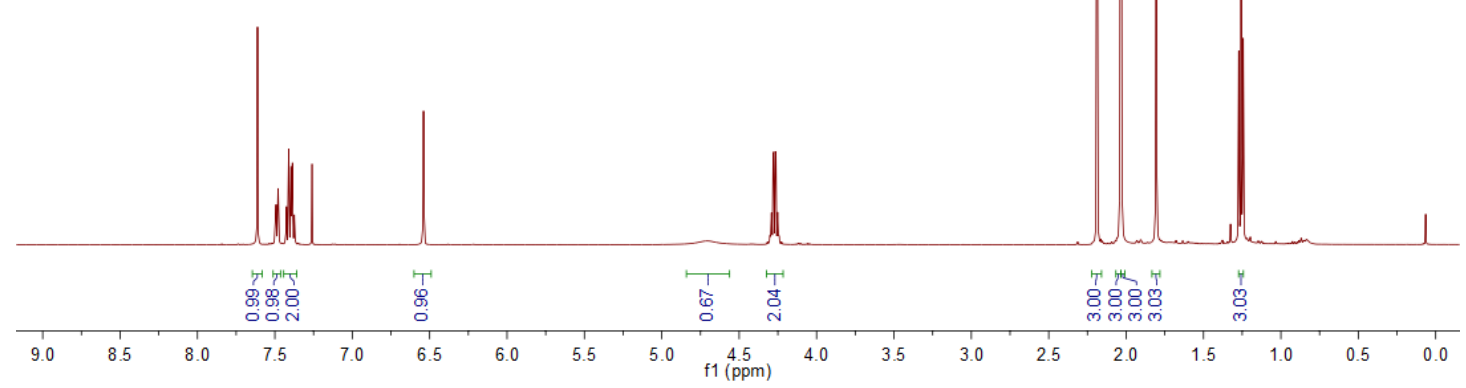

${ }^{13} \mathbf{C ~ N M R}\left(126 \mathrm{MHz}, \mathrm{CDCl}_{3}\right)$

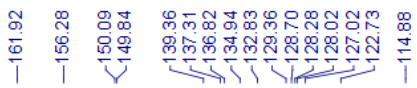

$\frac{\text { บ }}{\mathfrak{\leftarrow}}$

电电电

กิโ

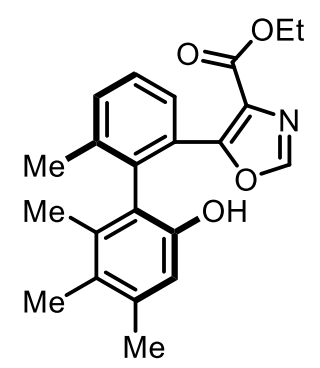

30

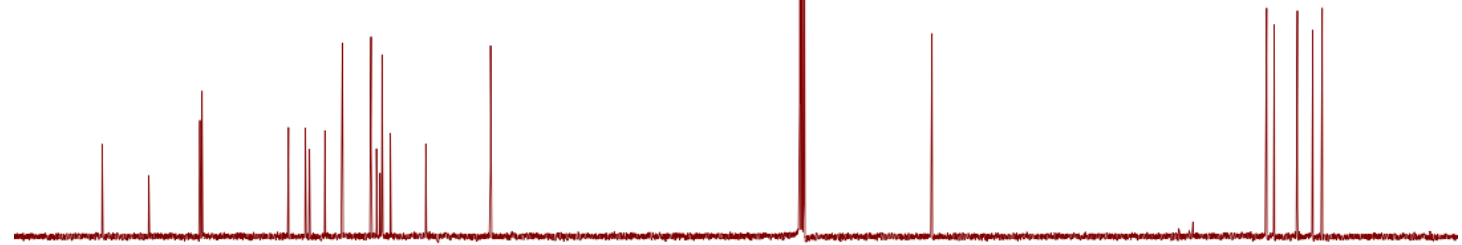

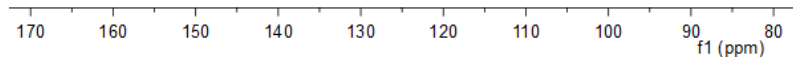


${ }^{1} \mathbf{H}$ NMR (500 MHz, $\left.\mathrm{CDCl}_{3}\right)$

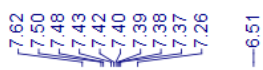<smiles>CCOC(=O)c1ncoc1-c1cccc(C)c1-c1c(O)cc([N+](=O)[O-])c(OC)c1OC</smiles>

${ }^{13} \mathbf{C ~ N M R}\left(126 \mathrm{MHz}, \mathrm{CDCl}_{3}\right)$

T<smiles>CCOC(=O)c1ncoc1-c1cccc(OC)c1-c1c(O)cc(C)c(OC)c1OC</smiles>

$3 p$

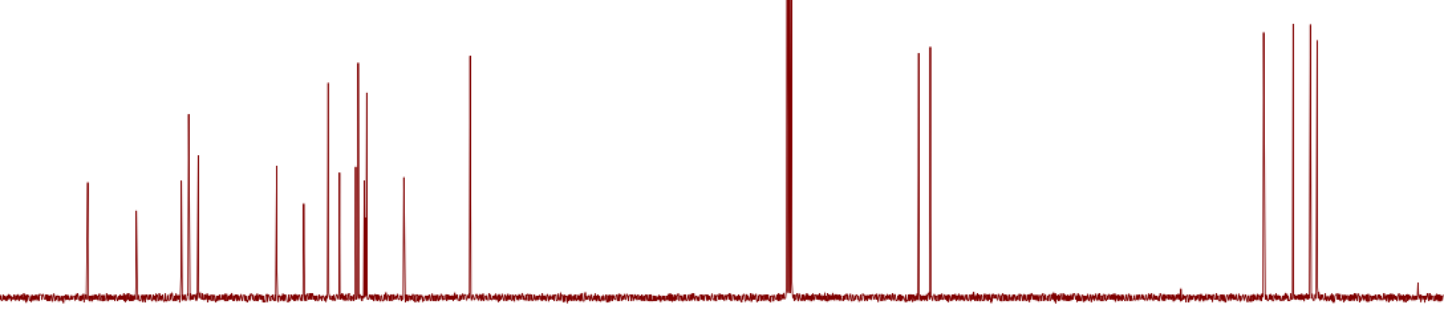

$$
\frac{1}{170}
$$

160

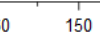

140

120

$9001(\mathrm{ppm})$ 
${ }^{1} \mathbf{H}$ NMR (500 MHz, $\left.\mathrm{CDCl}_{3}\right)$

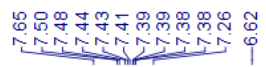<smiles>CCOC(=O)c1ncoc1-c1cccc(C)c1-c1c(O)cc([N+](=O)[O-])c(Cl)c1C</smiles>

$3 q$
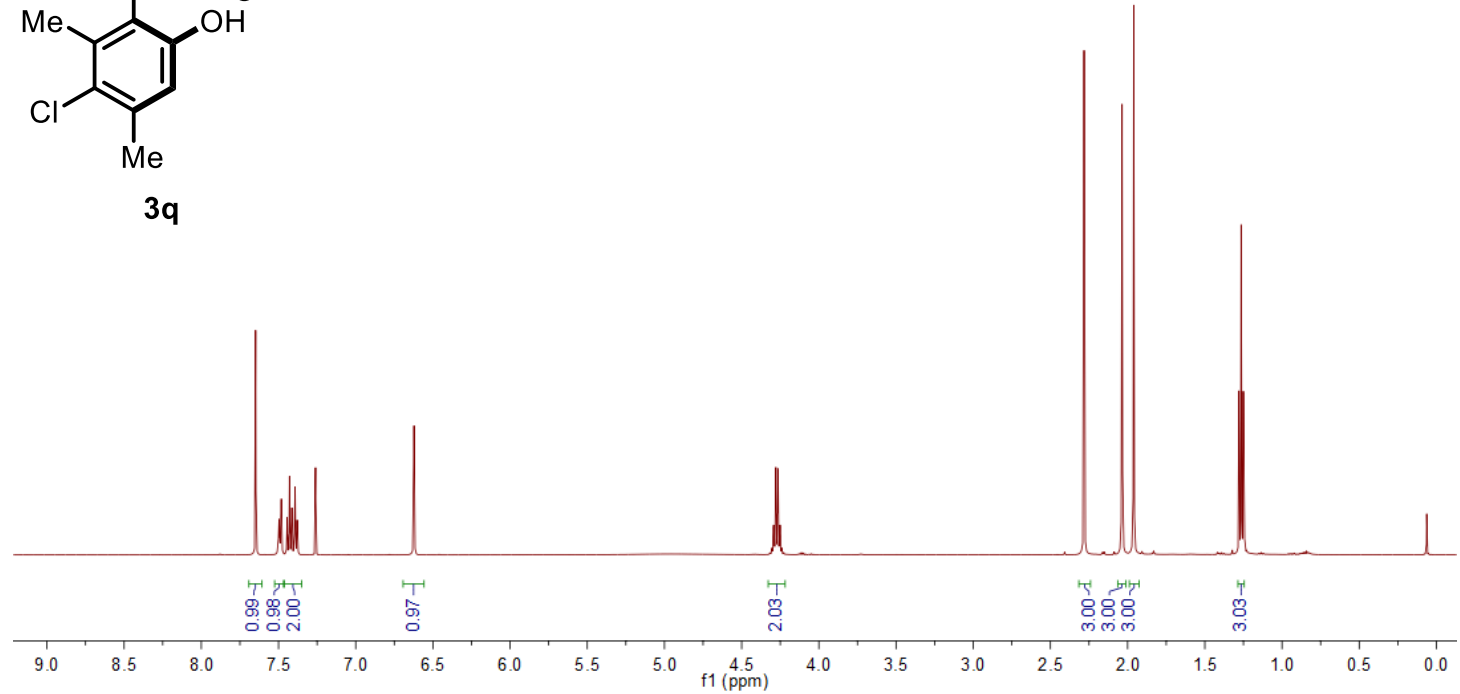

${ }^{13}$ C NMR (126 MHz, $\left.\mathrm{CDCl}_{3}\right)$

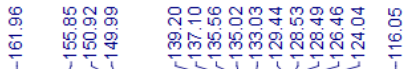

$\stackrel{\leftrightarrow}{i}$

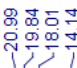<smiles>CCOC(=O)c1ncoc1-c1cccc(C)c1-c1c(O)cc(C)c(Cl)c1C</smiles>

$3 q$

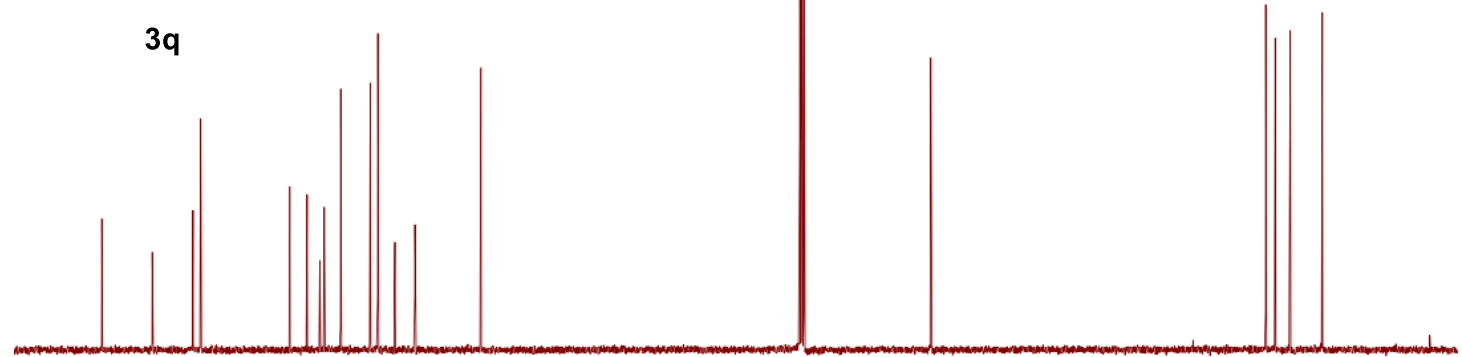

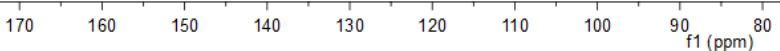


${ }^{1} \mathbf{H}$ NMR (500 MHz, $\left.\mathrm{CDCl}_{3}\right)$

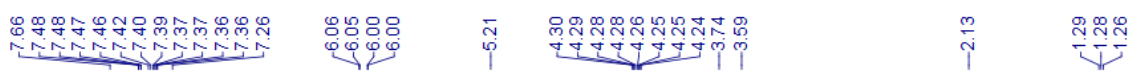<smiles>CCOC(=O)c1ncoc1-c1cccc(OC)c1-c1c(O)cc(OC)cc1OC</smiles>

$3 r$

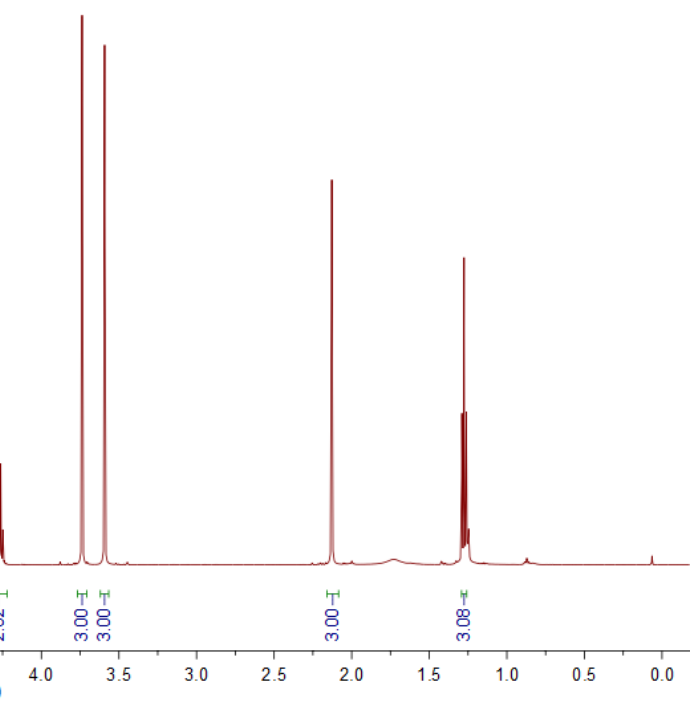

${ }^{13} \mathbf{C}$ NMR (126 MHz, $\left.\mathrm{CDCl}_{3}\right)$

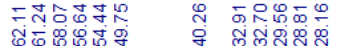

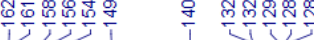

पर

OMe

$3 r$

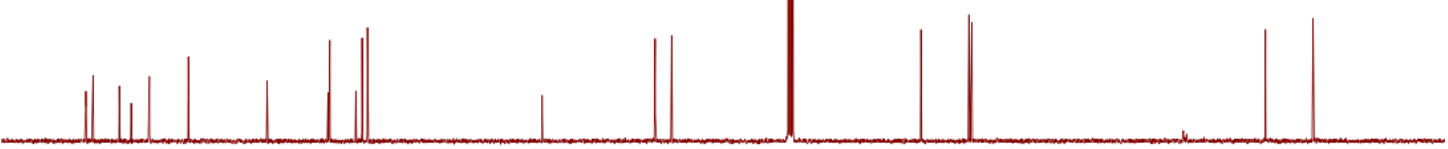

170

170

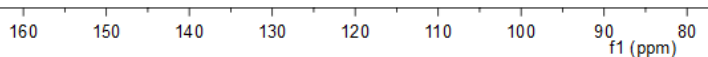


${ }^{1} \mathbf{H}$ NMR (400 MHz, $\left.\mathrm{CDCl}_{3}\right)$

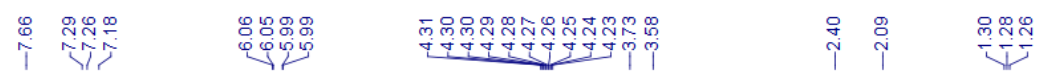<smiles>CCOC(=O)c1ncoc1-c1cc(C)cc(OC)c1-c1c(O)cc(OC)cc1OC</smiles>

3s

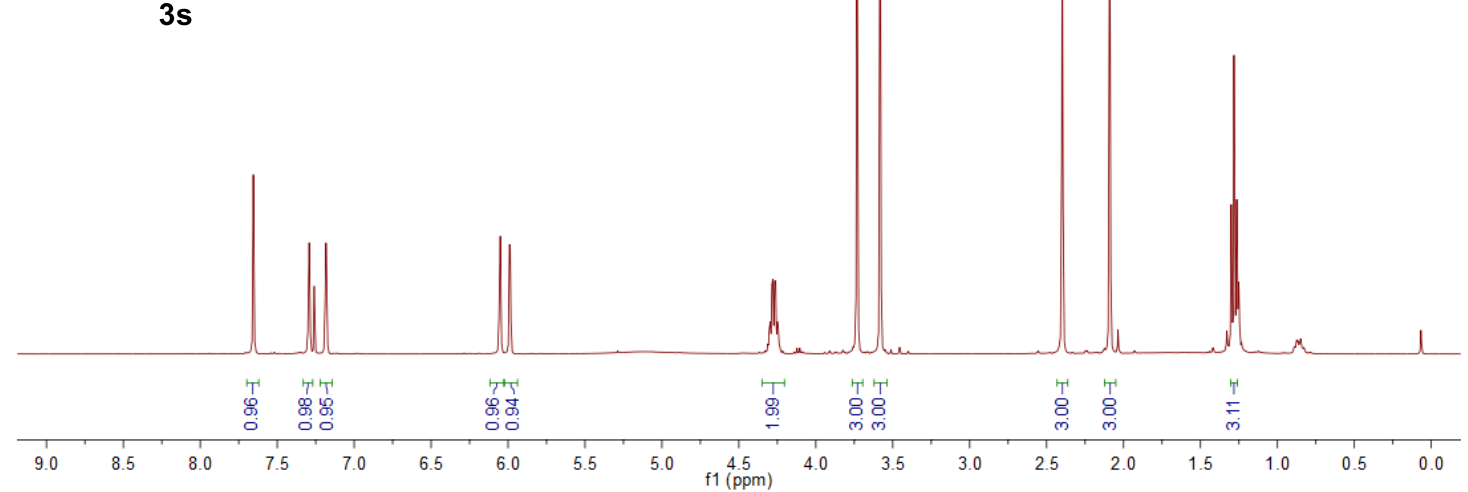

${ }^{13}$ C NMR (101 MHz, $\left.\mathrm{CDCl}_{3}\right)$

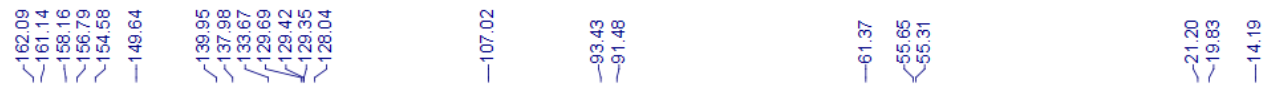<smiles>CCOC(=O)c1ncoc1-c1cc([N+](=O)[O-])cc(OC)c1-c1c(O)cc(OC)cc1OC</smiles>

3s

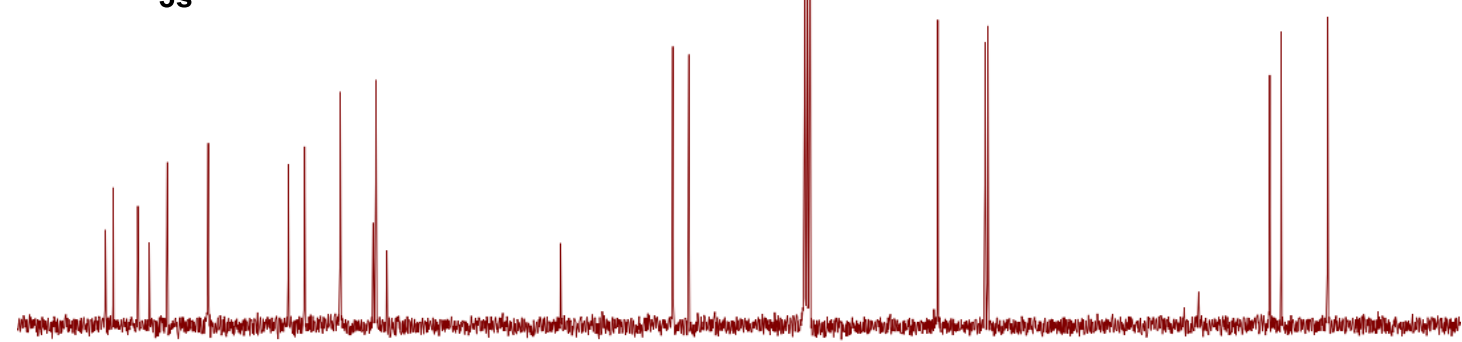

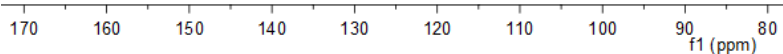


${ }^{1} \mathbf{H}$ NMR $\left(500 \mathrm{MHz}, \mathrm{CDCl}_{3}\right)$

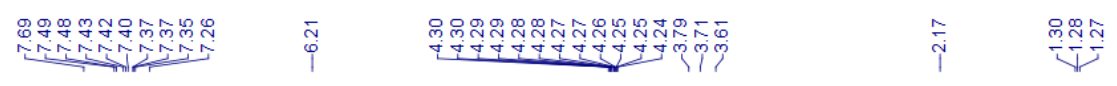<smiles>CCOC(=O)c1ncoc1-c1cccc(OC)c1-c1c(O)cc(OC)c(OC)c1OC</smiles>

3t

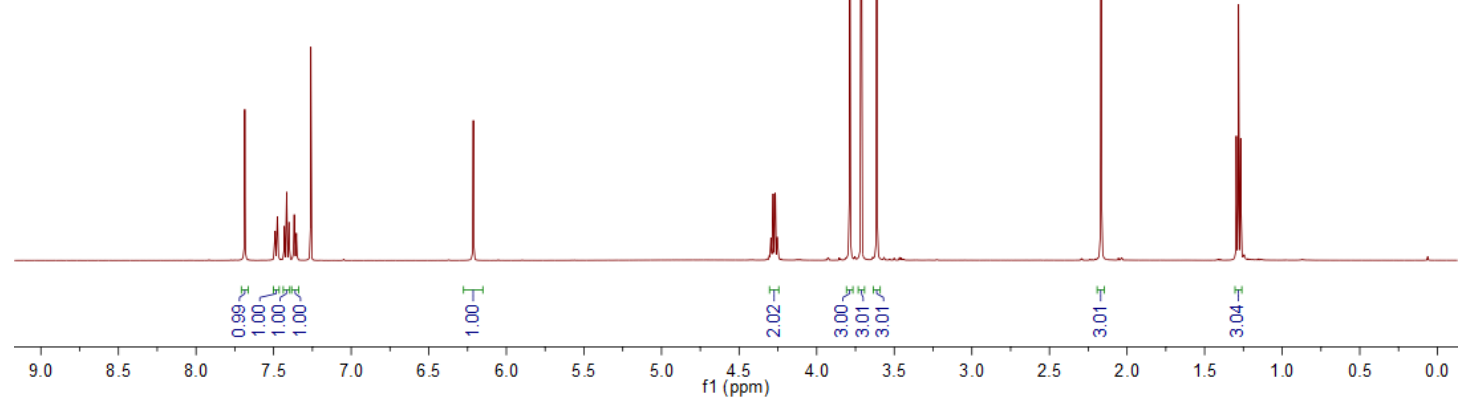

${ }^{13} \mathbf{C}$ NMR $\left(126 \mathrm{MHz}, \mathrm{CDCl}_{3}\right)$

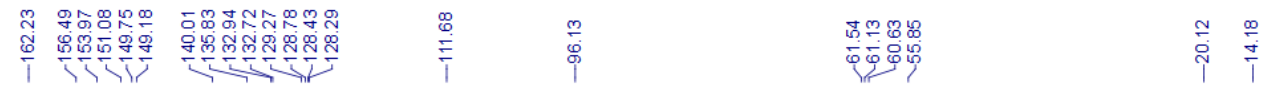<smiles>CCOC(=O)c1ncoc1-c1cccc(OC)c1-c1c(O)cc(OC)c(OC)c1OC</smiles>

$3 t$

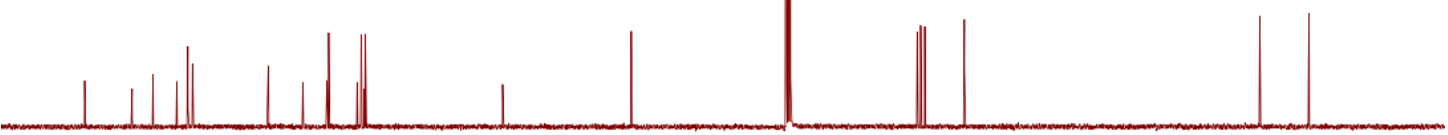

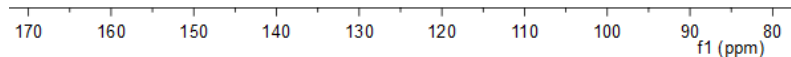


${ }^{1} \mathbf{H}$ NMR (500 MHz, $\left.\mathrm{CDCl}_{3}\right)$

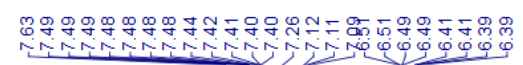

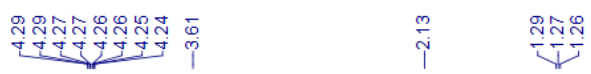

(1)
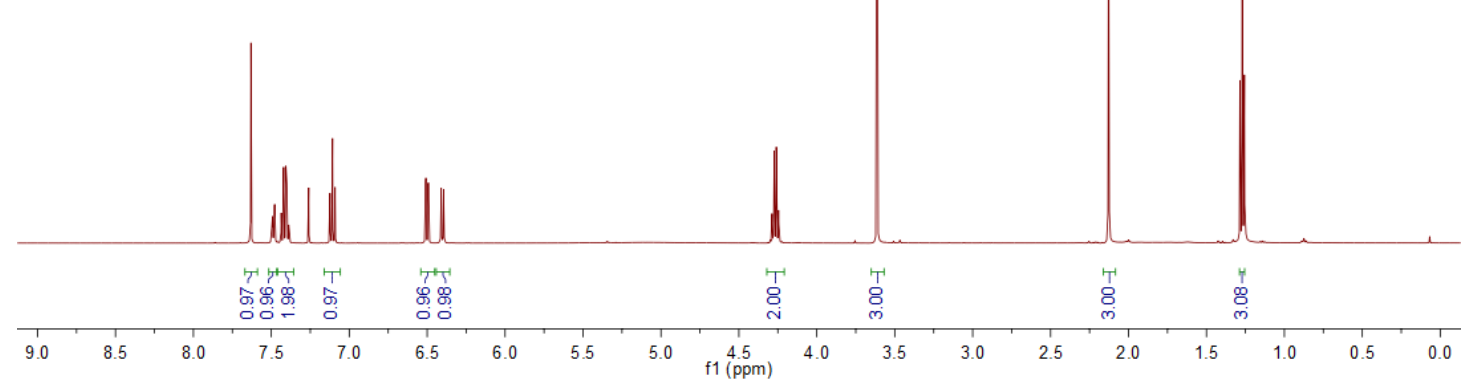

${ }^{13} \mathbf{C}$ NMR (126 MHz, $\left.\mathrm{CDCl}_{3}\right)$

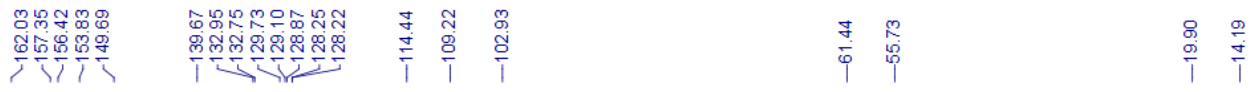<smiles>CCOC(=O)c1ncoc1-c1cccc(OC)c1-c1c(O)cccc1OC</smiles>

$3 u$

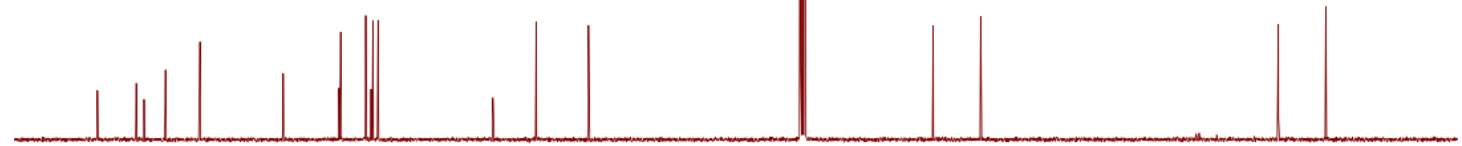

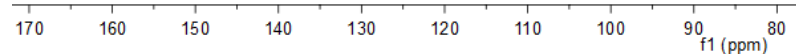


${ }^{1} \mathbf{H}$ NMR (500 MHz, $\left.\mathrm{CDCl}_{3}\right)$

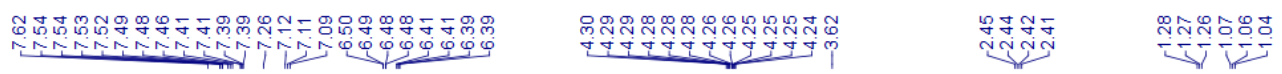<smiles>CCOC(=O)c1ncoc1-c1cccc(CC)c1-c1c(O)cccc1OC</smiles>

${ }^{13} \mathbf{C}$ NMR (126 MHz, $\left.\mathrm{CDCl}_{3}\right)$

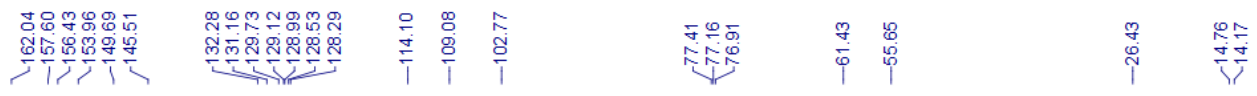<smiles>CCOC(=O)c1ncoc1-c1cccc(OC)c1-c1c(C)cccc1O</smiles>

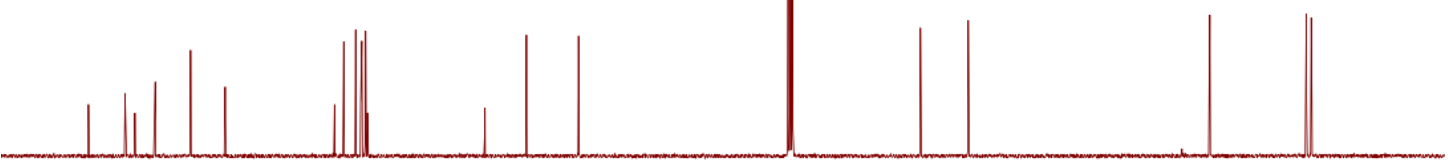

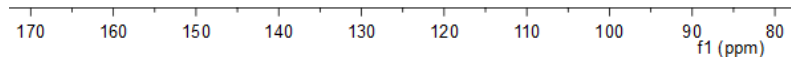


${ }^{1} \mathbf{H}$ NMR (500 MHz, $\left.\mathrm{CDCl}_{3}\right)$

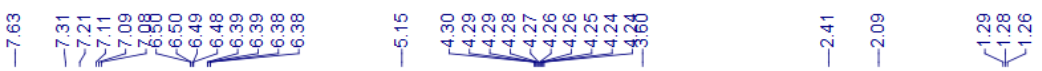<smiles>CCOC(=O)c1ncoc1-c1cc(C(=O)O)cc(OC)c1-c1c(O)cccc1O</smiles>

${ }^{13}$ C NMR (126 MHz, $\left.\mathrm{CDCl}_{3}\right)$

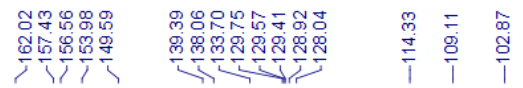

\begin{tabular}{ll}
$\infty$ & $?$ \\
0 & 0 \\
\hline 0 & 0 \\
\hline & 0
\end{tabular}

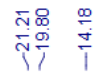<smiles>CCOC(=O)c1ncoc1-c1cc(C(=O)O)cc(OC)c1-c1c(O)cccc1O</smiles>

$3 w$

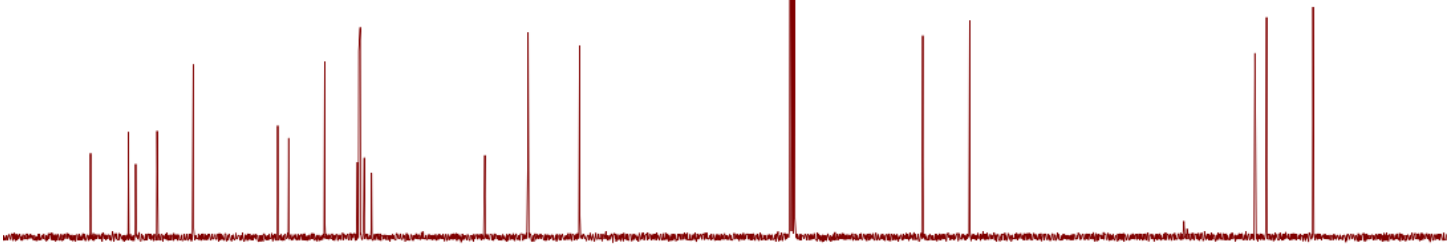

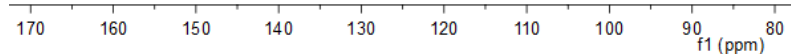


${ }^{1} \mathbf{H}$ NMR (500 MHz, $\left.\mathrm{CDCl}_{3}\right)$

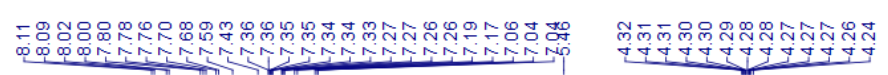

(1)

$3 x$

${ }^{13} \mathbf{C ~ N M R}\left(126 \mathrm{MHz}, \mathrm{CDCl}_{3}\right)$

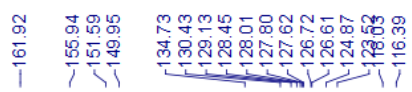

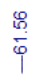

$\stackrel{8}{i}$<smiles>CCOC(=O)c1ncoc1-c1ccc2ccccc2c1-c1c(O)ccc2ccccc12</smiles>

$3 x$
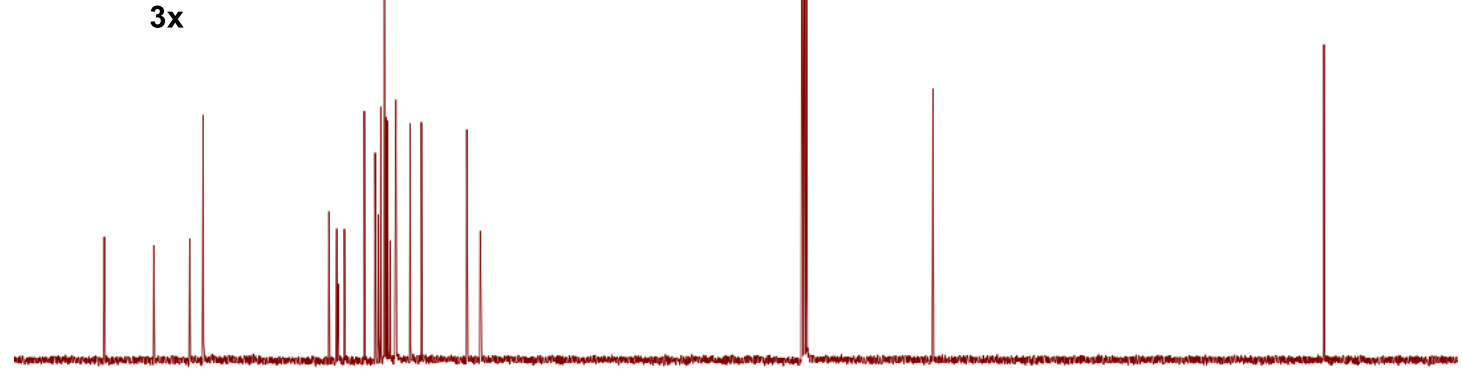

17

160

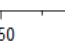

$140 \quad 130$

${ }_{90}^{1}(\mathrm{ppm})$ 
${ }^{1} \mathbf{H}$ NMR $\left(500 \mathrm{MHz}, \mathrm{CDCl}_{3}\right)$

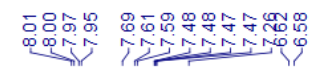

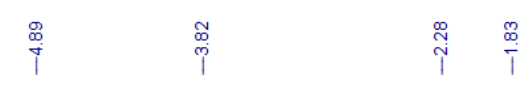<smiles>COC(=O)c1ncoc1-c1ccc2ccccc2c1-c1c(C)cc(C)cc1O</smiles>

$4 a$

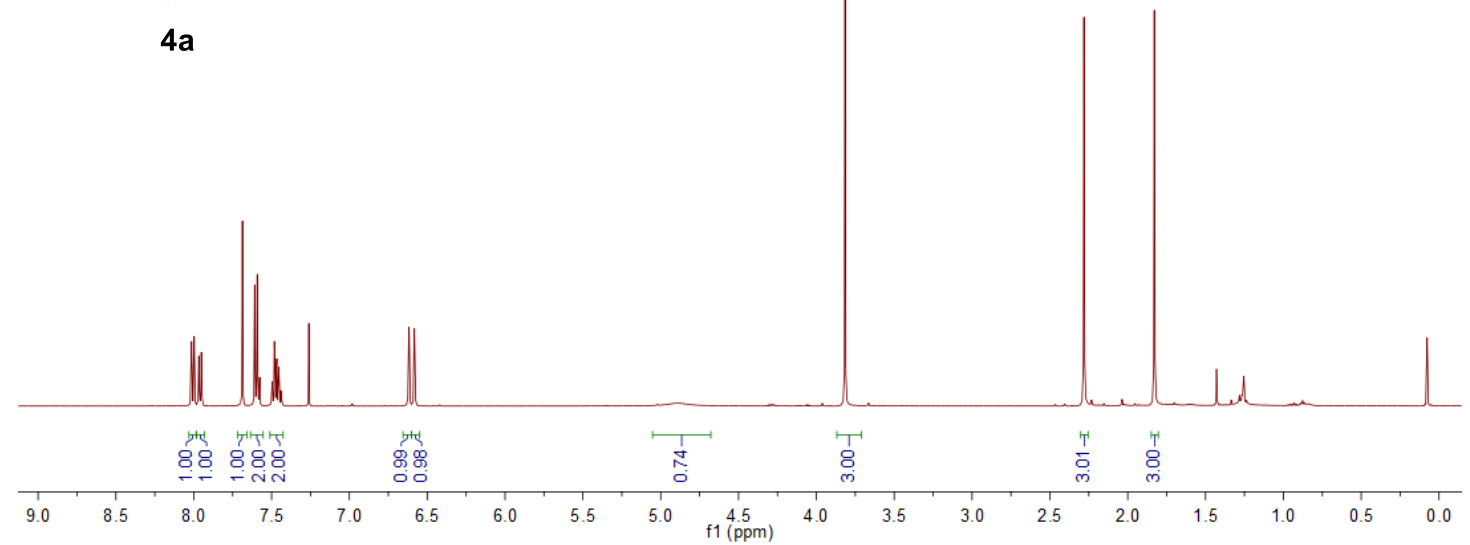

${ }^{13}$ C NMR (126 MHz, $\left.\mathrm{CDCl}_{3}\right)$

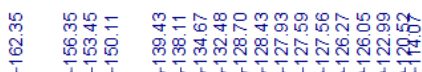
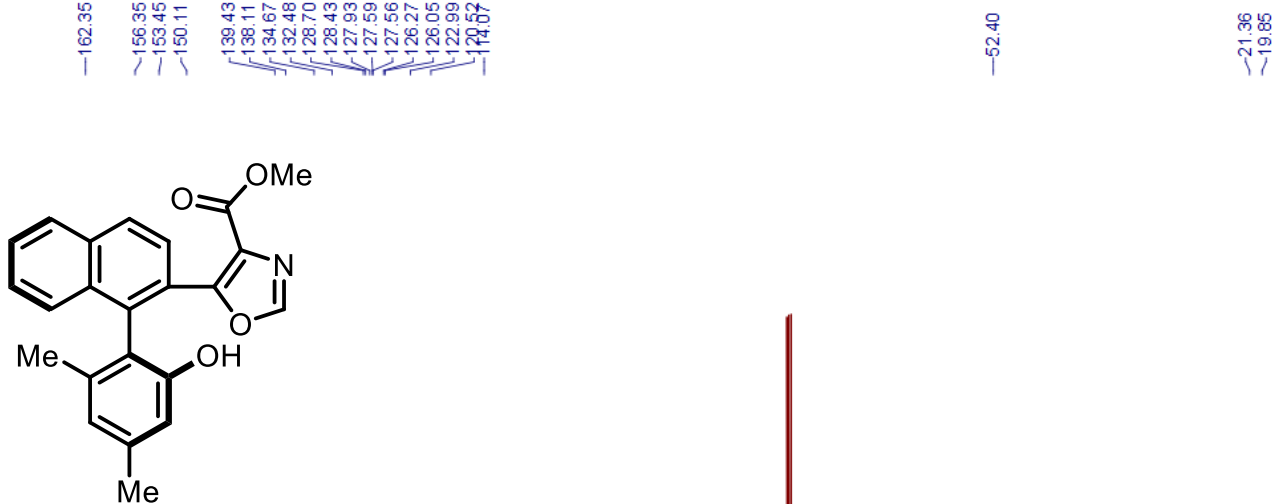

$4 a$

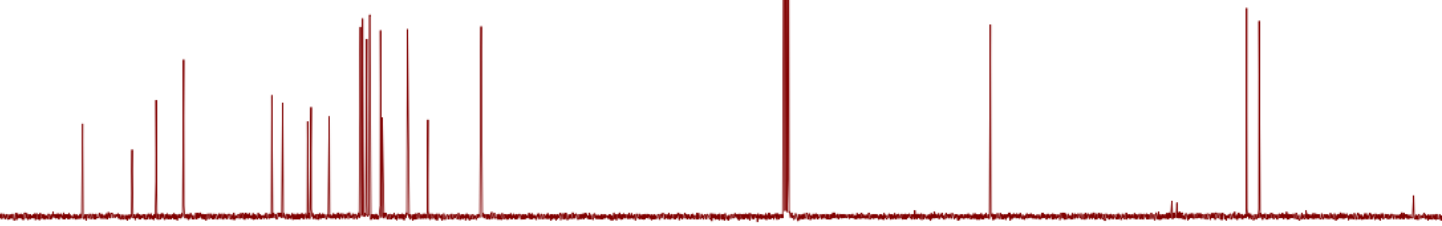

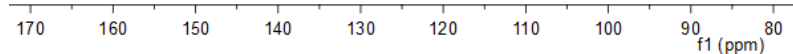


${ }^{1} \mathbf{H}$ NMR $\left(500 \mathrm{MHz}, \mathrm{CDCl}_{3}\right)$

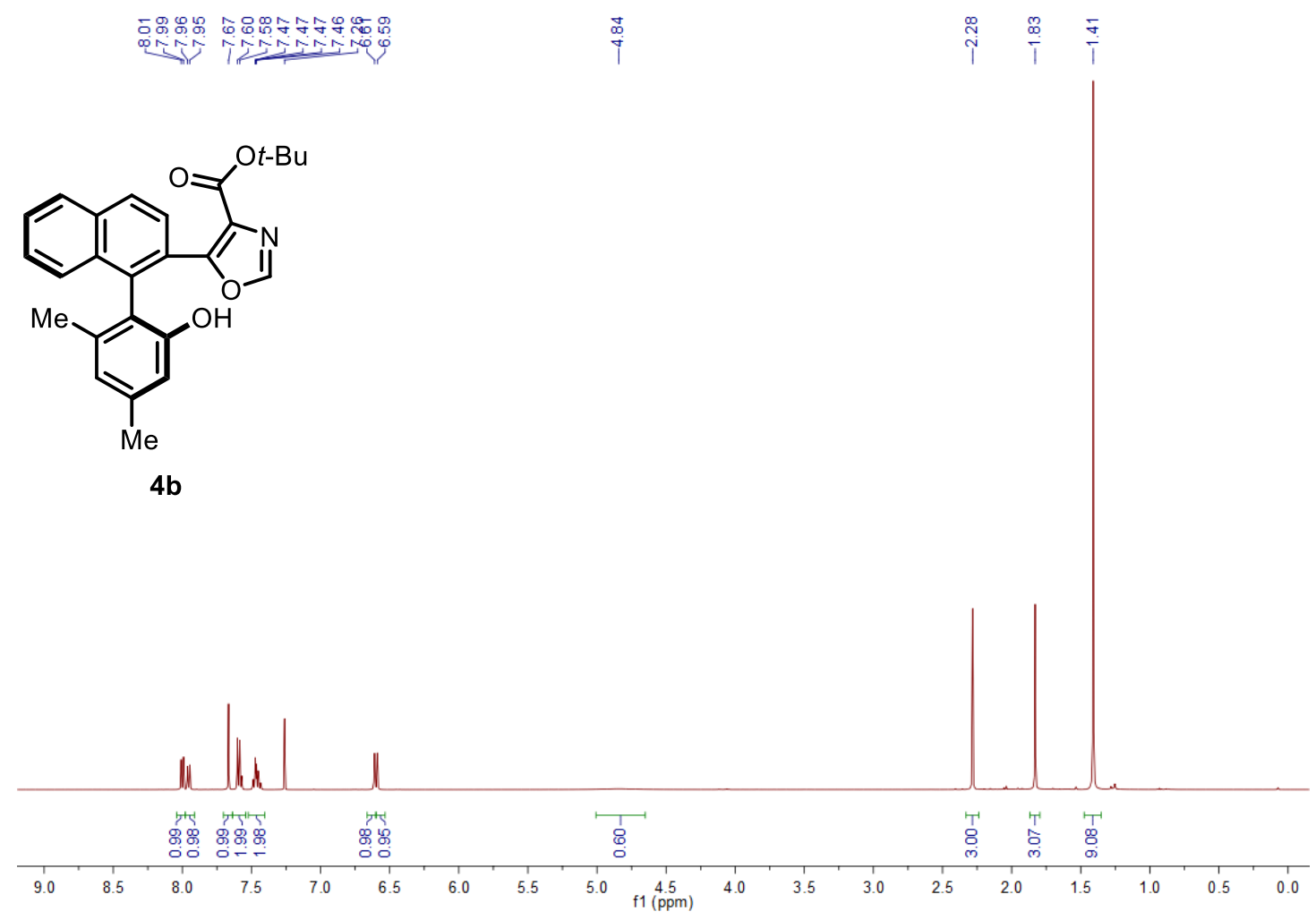

${ }^{13} \mathbf{C}$ NMR $\left(126 \mathrm{MHz}, \mathrm{CDCl}_{3}\right)$

0
0
0
0

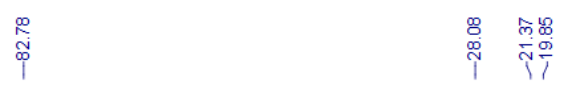

(1)

4b

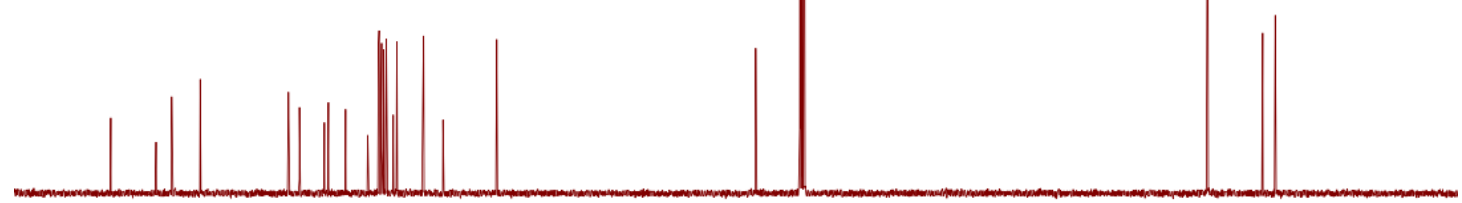

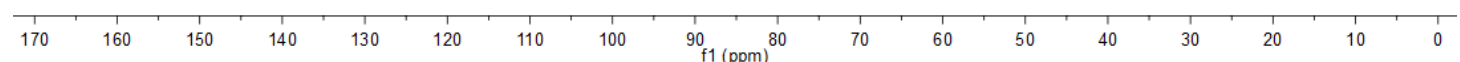


${ }^{1} \mathbf{H}$ NMR $\left(500 \mathrm{MHz}, \mathrm{CDCl}_{3}\right)$

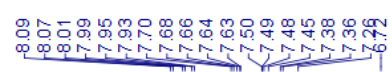

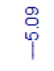<smiles>Cc1ccc(S(=O)(=O)c2ncoc2-c2ccc3ccccc3c2-c2c(O)cc([N+](=O)[O-])c(Cl)c2C)cc1</smiles>

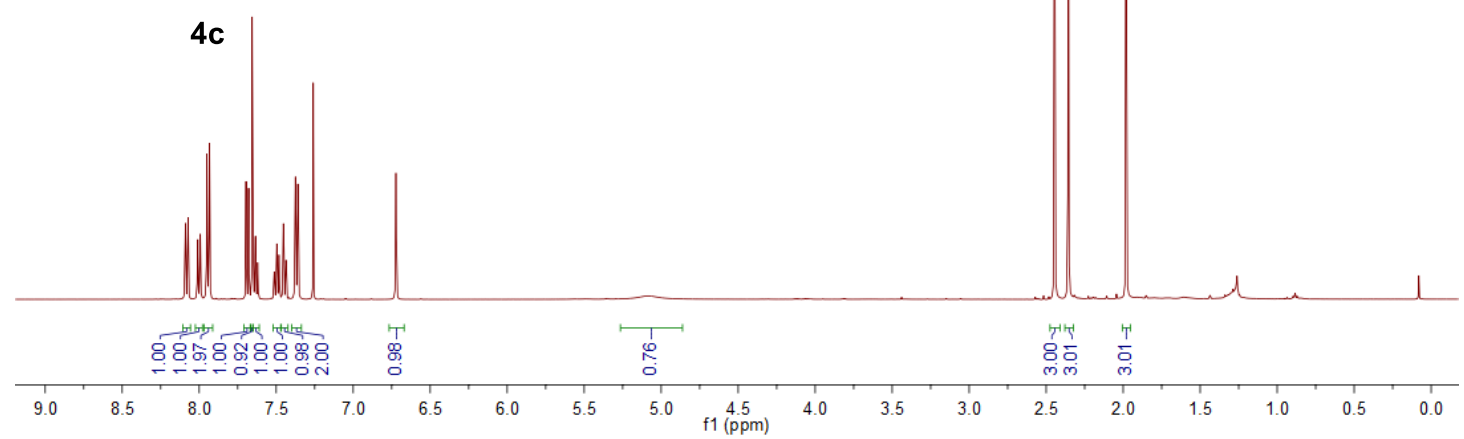

${ }^{13} \mathbf{C}$ NMR (126 MHz, $\left.\mathrm{CDCl}_{3}\right)$

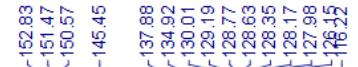

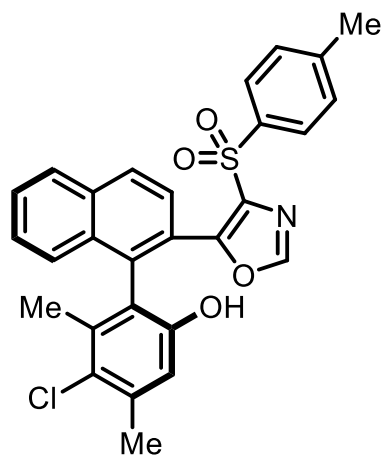

$4 c$

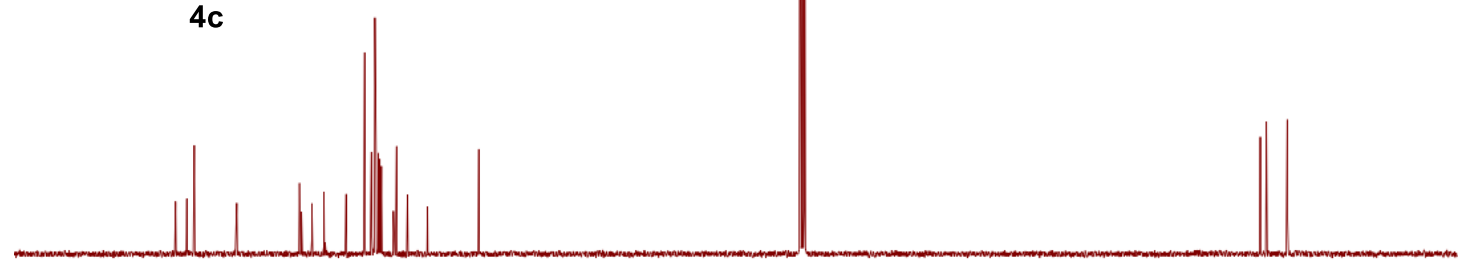

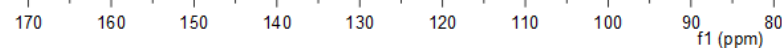


${ }^{1} \mathbf{H}$ NMR (500 MHz, $\left.\mathrm{CDCl}_{3}\right)$

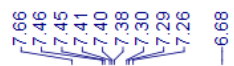

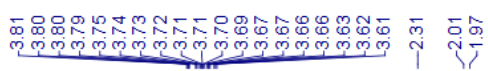<smiles>Cc1cccc(-c2ocnc2C(=O)N2CCOCC2)c1-c1c(O)cc([N+](=O)[O-])c(Cl)c1C</smiles>

4d

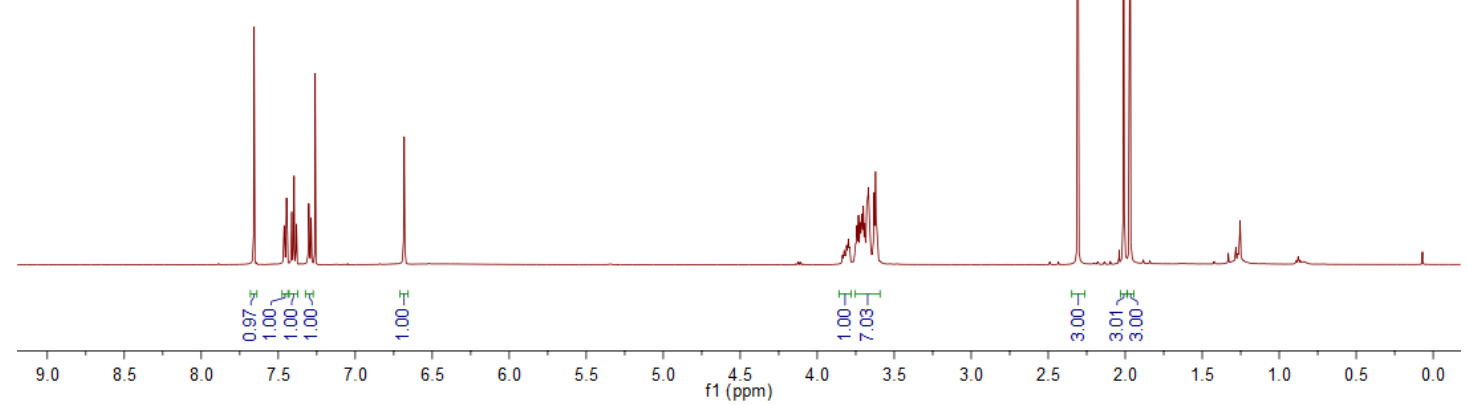

${ }^{13} \mathbf{C}$ NMR $\left(126 \mathrm{MHz}, \mathrm{CDCl}_{3}\right)$

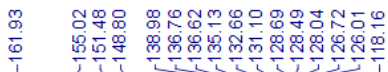

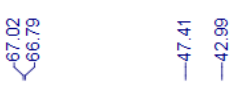

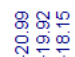<smiles>COc1cc(O)c(-c2c(C)cccc2-c2ocnc2C(=O)N2CCOCC2)c(C)c1Cl</smiles>

4d

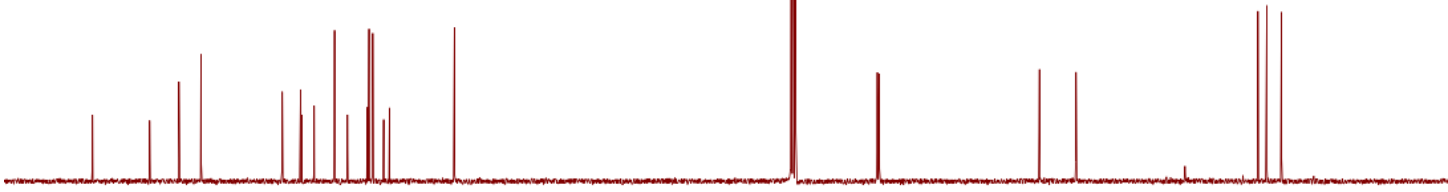

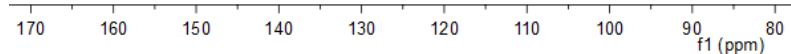


${ }^{1}$ H NMR (500 MHz, DMSO)

0
0

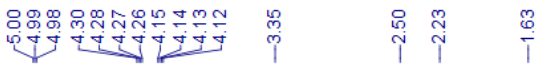

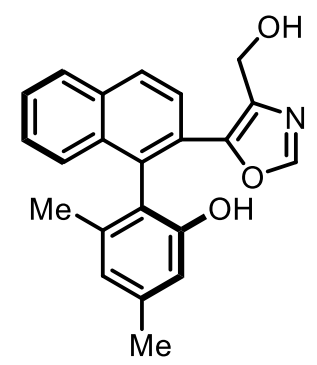

5

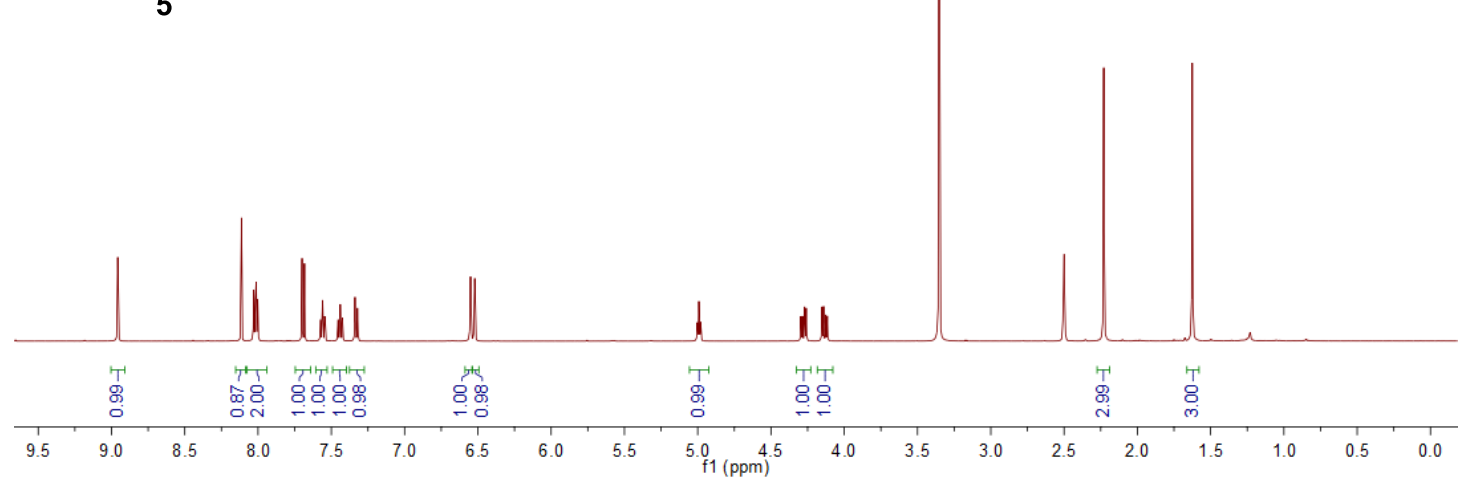

${ }^{13}$ C NMR (126 MHz, DMSO)

कर

5눈
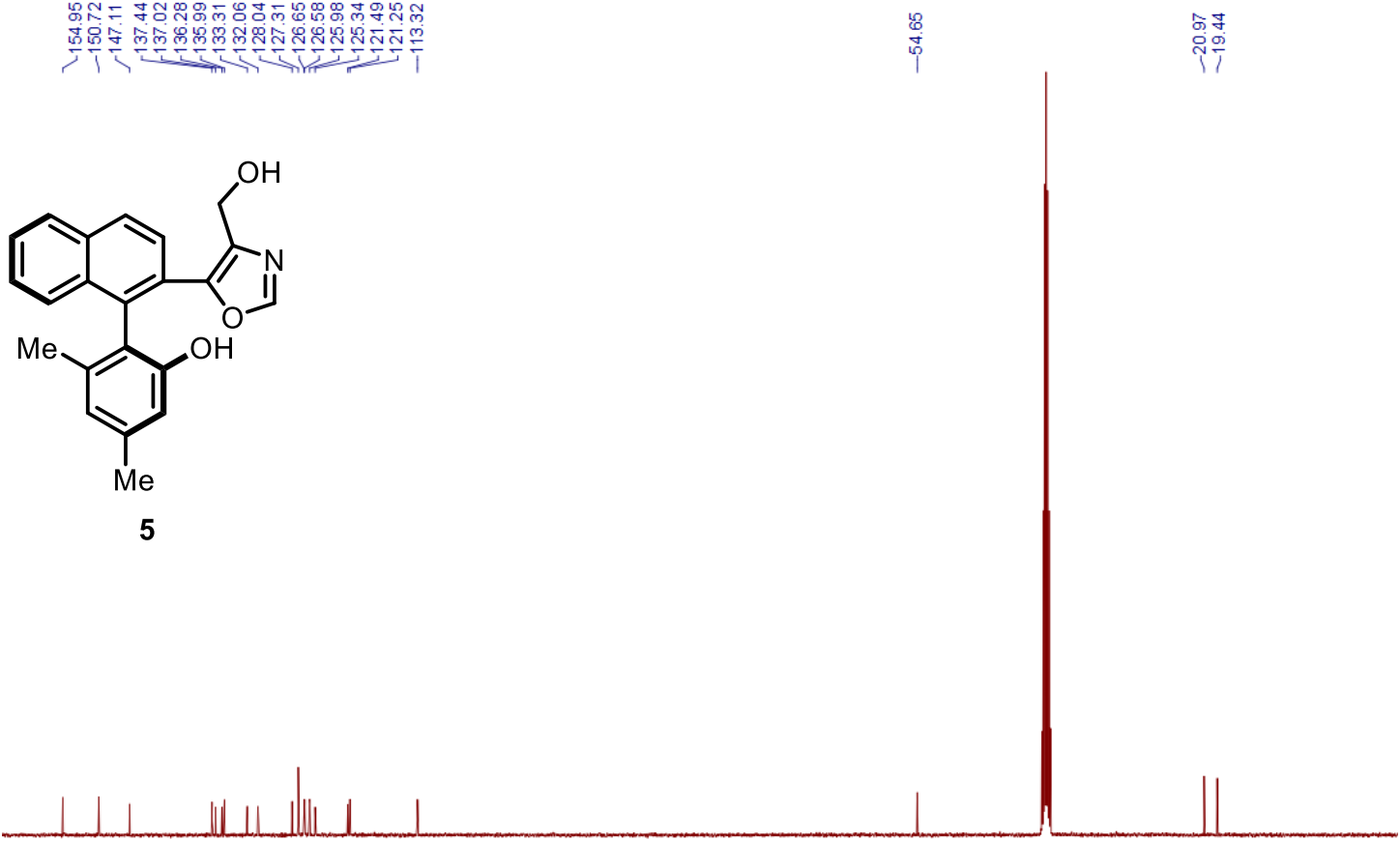

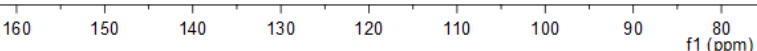


${ }^{1} \mathbf{H}$ NMR $\left(500 \mathrm{MHz}, \mathrm{CDCl}_{3}\right)$

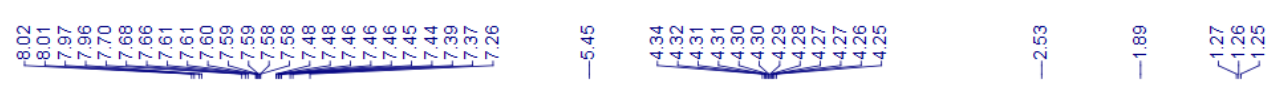<smiles>CCOC(=O)c1ncoc1-c1c(O)c(Cl)c([N+](=O)[O-])c(Cl)c1C1C(=O)C=Cc2ccccc21</smiles>

6

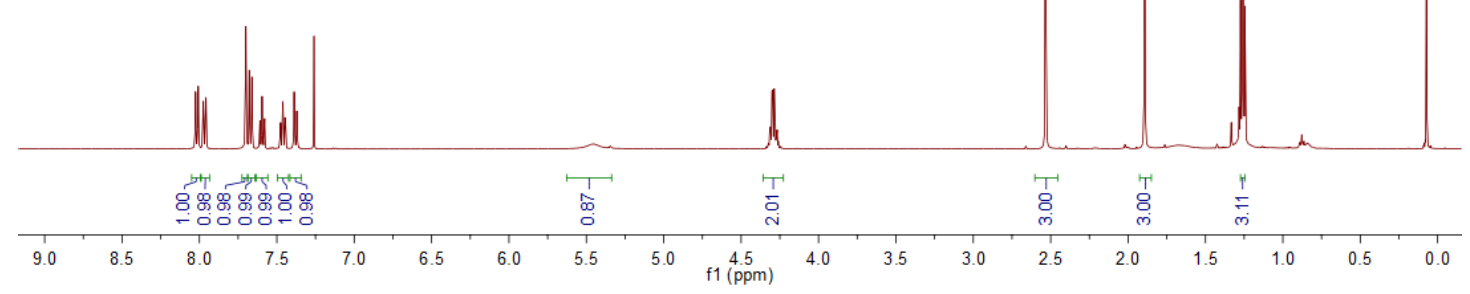

${ }^{13} \mathbf{C}$ NMR (126 MHz, $\left.\mathrm{CDCl}_{3}\right)$

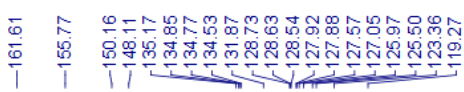

$\frac{\widetilde{\varphi}}{\bar{\varphi}}$

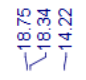<smiles>CCOC(=O)c1ncoc1-c1c(O)c(Cl)c([N+](=O)[O-])c(Cl)c1O</smiles>

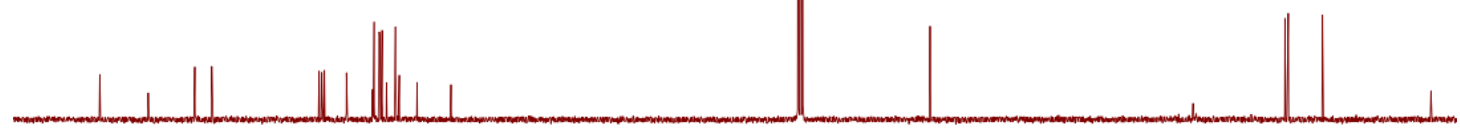

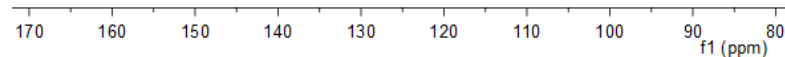


${ }^{1} \mathbf{H}$ NMR $\left(500 \mathrm{MHz}, \mathrm{CDCl}_{3}\right)$

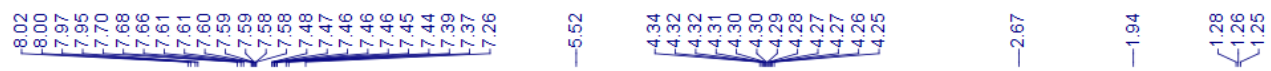<smiles>CCOC(=O)c1ncoc1-c1ccc2ccccc2c1-c1c(O)c(Br)c([N+](=O)[O-])c(Br)c1Br</smiles>

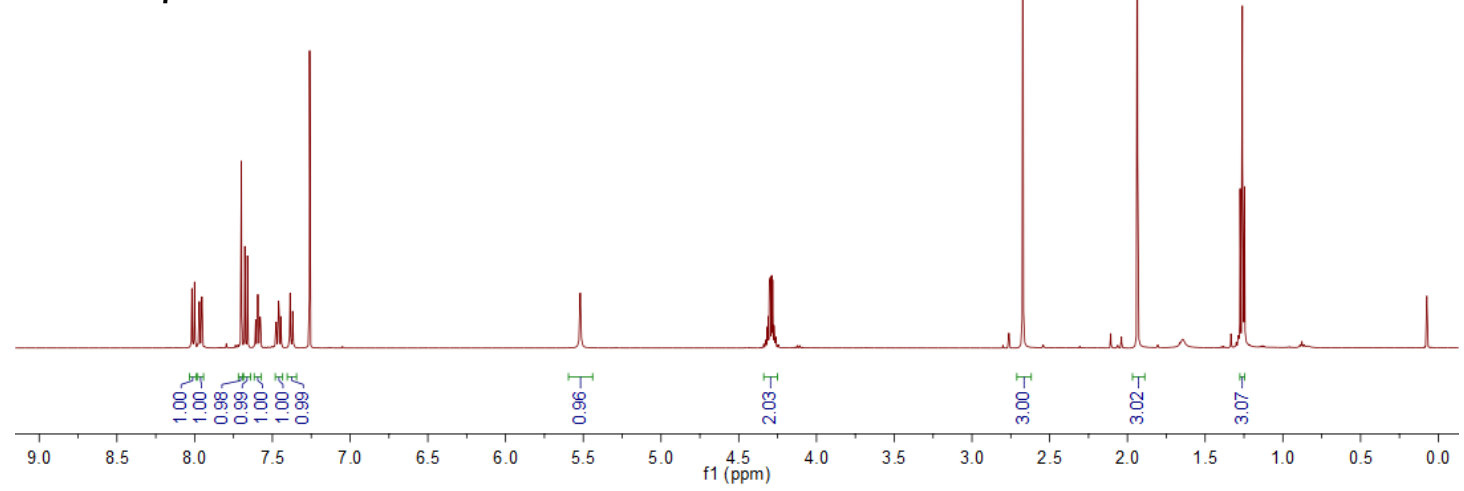

${ }^{13} \mathbf{C}$ NMR (126 MHz, $\left.\mathrm{CDCl}_{3}\right)$

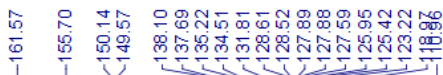

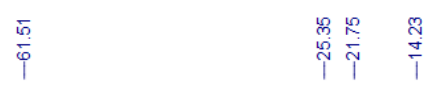<smiles>CCOC(=O)c1ncoc1-c1c(O)c(Br)c([N+](=O)[O-])c(Br)c1Cc1ccc2ccccc2c1</smiles>

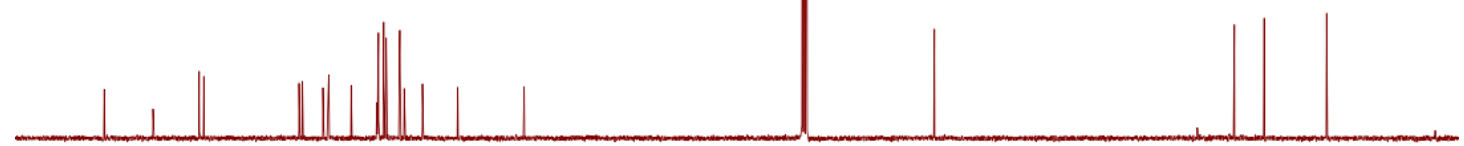

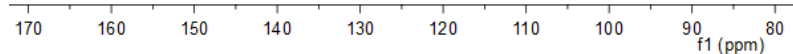


${ }^{1} \mathbf{H}$ NMR (400 MHz, $\left.\mathrm{CDCl}_{3}\right)$

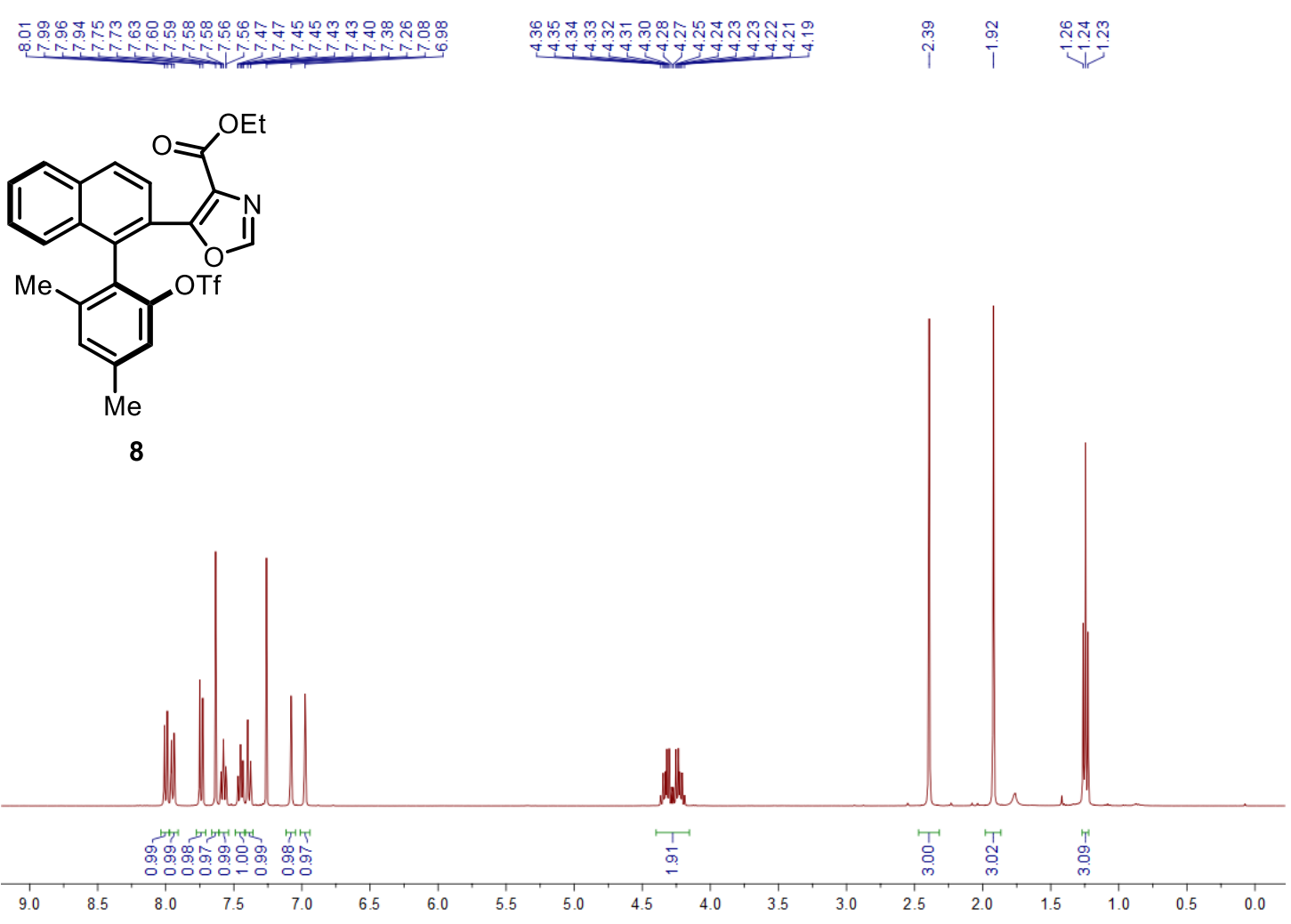

${ }^{13}$ C NMR (101 MHz, $\left.\mathrm{CDCl}_{3}\right)$

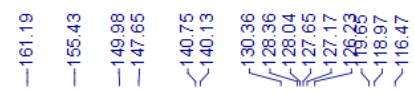<smiles>CCOC(=O)c1ncoc1-c1ccc2ccccc2c1-c1c(C)cc([N+](=O)[O-])cc1C</smiles>

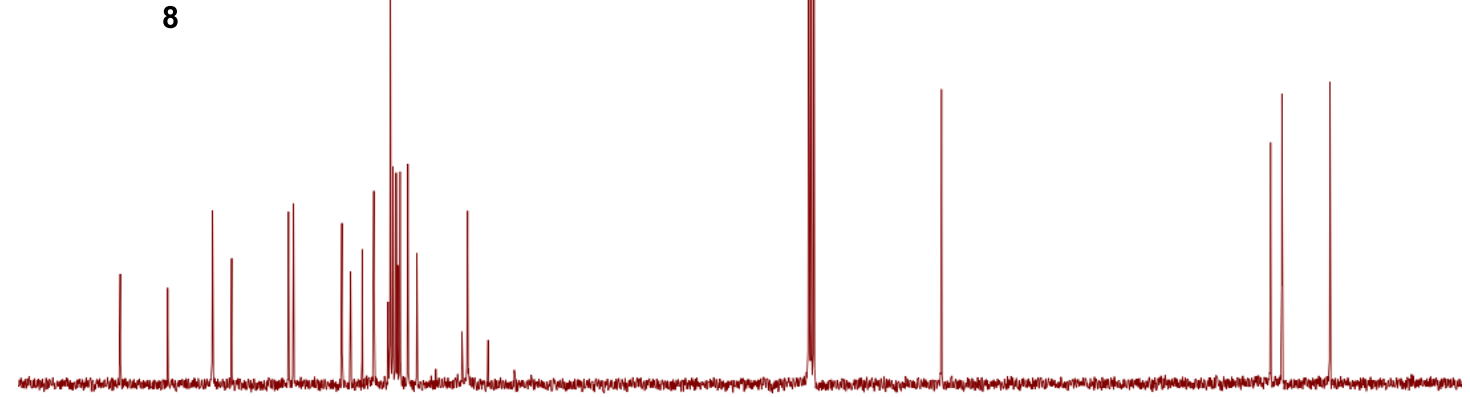

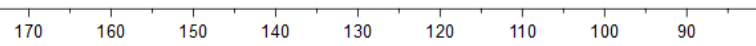


${ }^{1}$ H NMR (400 MHz, DMSO)

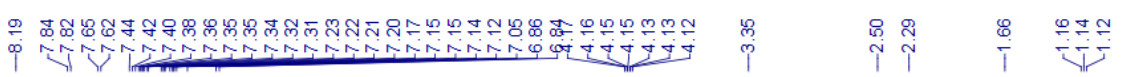<smiles>CCOC(=O)c1ncoc1-c1ccc2ccccc2c1-c1c(C)cc(C)cc1P(=O)(O)O</smiles>

9
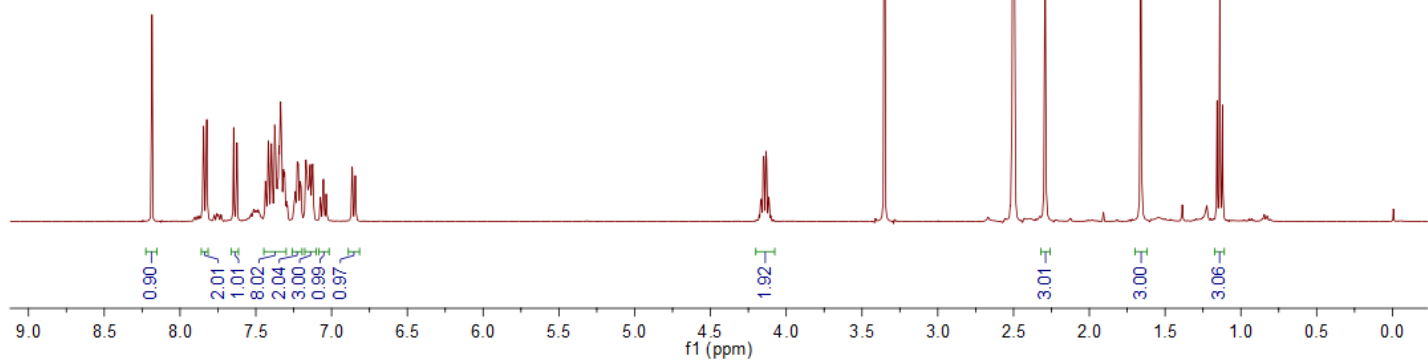

${ }^{13}$ C NMR (101 MHz, DMSO)

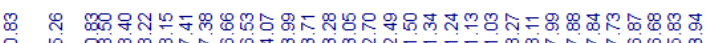

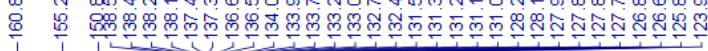

1 (1)<smiles>CCOC(=O)c1ncoc1-c1ccc2ccccc2c1-c1c([N+](=O)[O-])cc([N+](=O)[O-])cc1[N+](=O)[O-]</smiles>

9
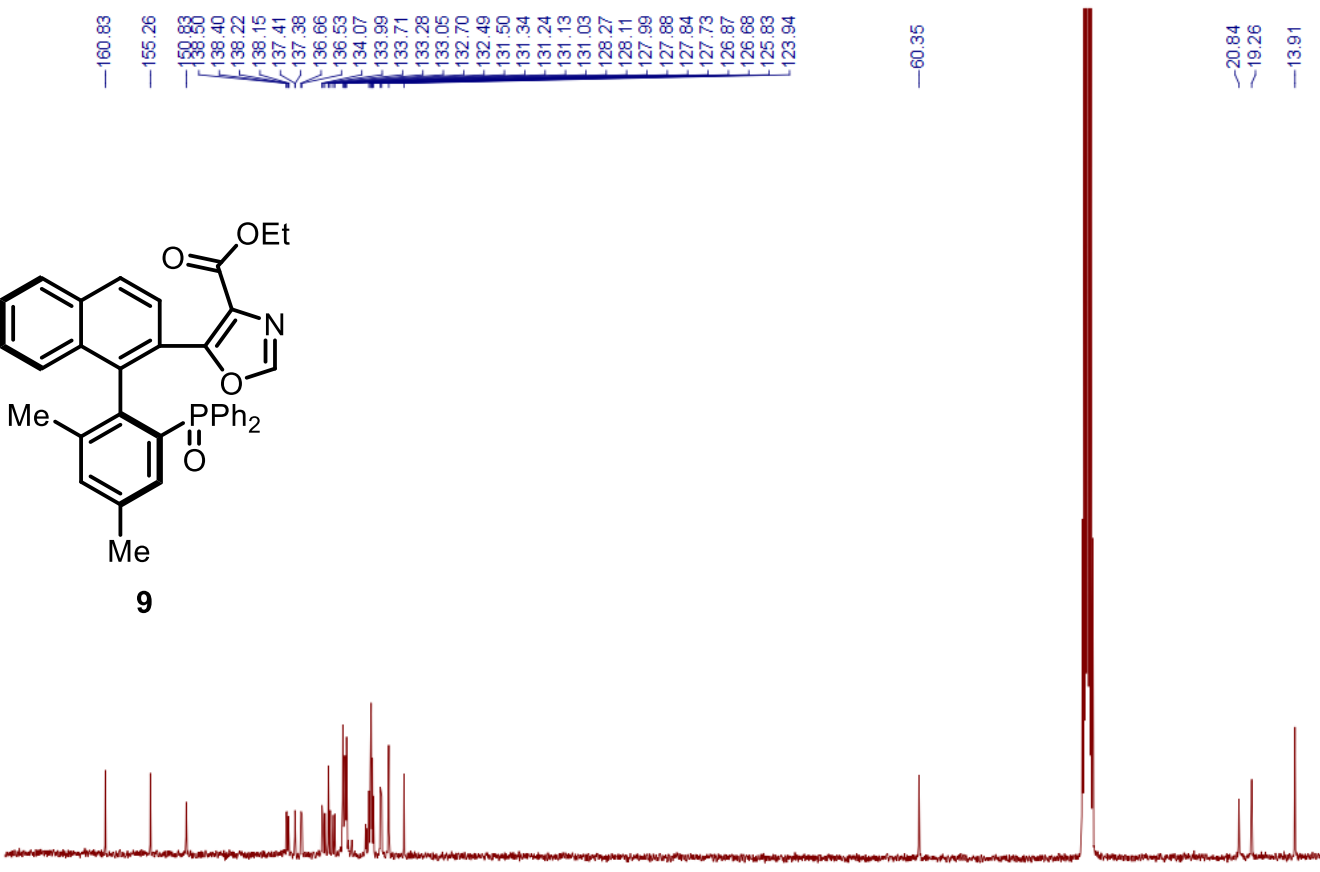

\begin{tabular}{lllllllll}
\hline 170 & 160 & 150 & 140 & 130 & 120 & 110 & 100 & $\underset{\mathrm{f} 1(\mathrm{ppm})}{1} 80$
\end{tabular} 
${ }^{1} \mathbf{H}$ NMR (500 MHz, $\left.\mathrm{CDCl}_{3}\right)$

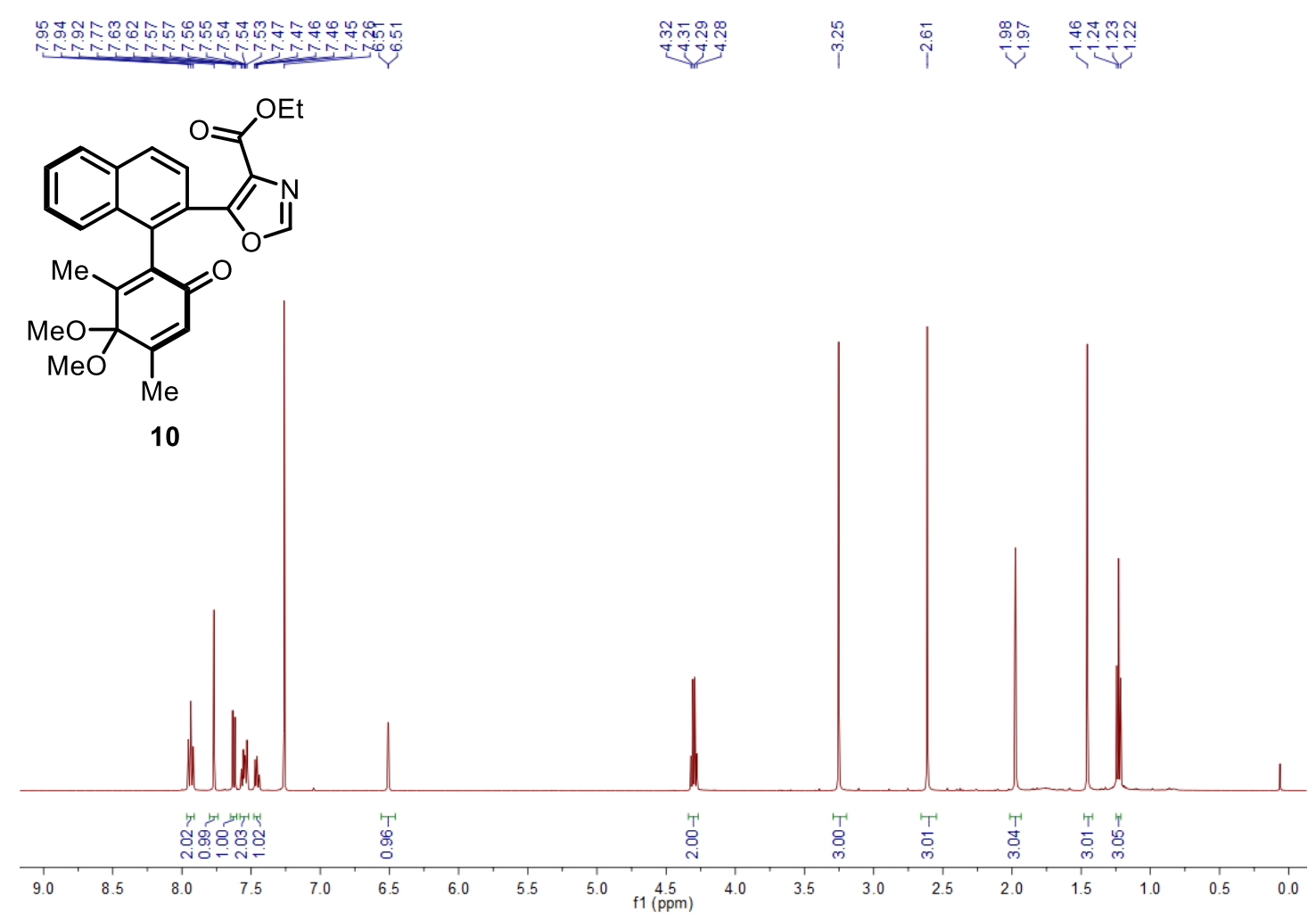

${ }^{13} \mathbf{C ~ N M R}\left(126 \mathrm{MHz}, \mathrm{CDCl}_{3}\right)$

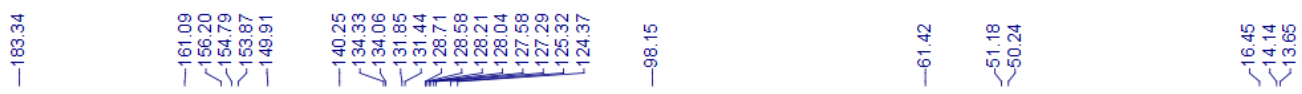<smiles>CCOC(=O)c1ncoc1-c1ccc2ccccc2c1C1=C(O)C(OC)(OC)C(C)=CC1=O</smiles>

10

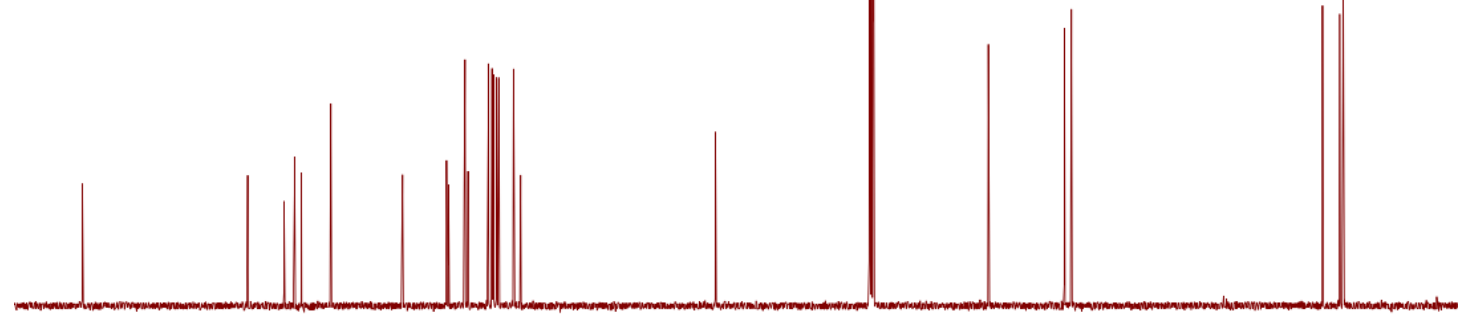

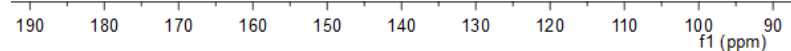


${ }^{1} \mathbf{H}$ NMR (500 MHz, $\left.\mathrm{CDCl}_{3}\right)$

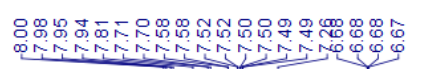

过等主

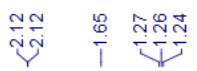

(1)

11

${ }^{13}$ C NMR (126 MHz, $\left.\mathrm{CDCl}_{3}\right)$

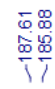

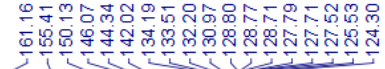

$\frac{f}{9}$

产舀品<smiles>CCOC(=O)c1ncoc1-c1ccc2ccccc2c1C1=C(C)C(=O)C(C)=CC1=O</smiles>

11

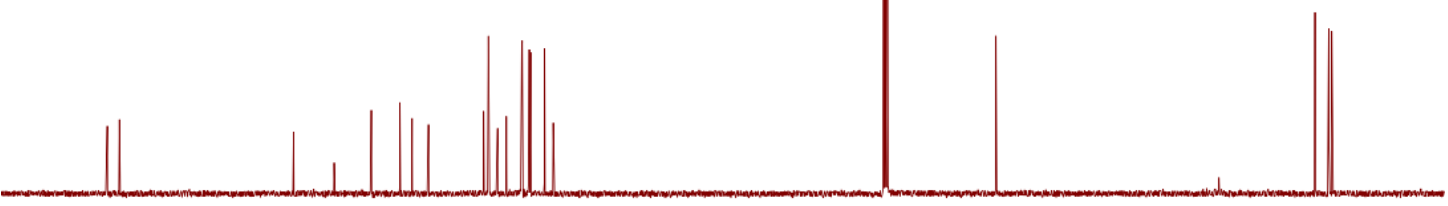

\begin{tabular}{llllllllll}
\hline 200 & 190 & 180 & 170 & 160 & 150 & 140 & 130 & 120 & 110 \\
$\mathrm{f} 1(\mathrm{ppm})$
\end{tabular} 